\title{
Highly Selective Cascade Couplings for the Syntheses of Functionalized Piperidinones and Bispidines
}

\author{
Feng Xu,*, Edward Corley, Jerry Murry, David M. Tschaen \\ Department of Process Research, Merck Research Laboratories, Rahway, NJ 07065, USA \\ E-mail:feng_xu@merck.com
}

\section{Supporting Information}

\section{$\underline{\text { Table of Contents }}$}

1. Procedure for the one-pot preparation of trans pyrrolidinones $1 \ldots \ldots \ldots \ldots \ldots \ldots . . . . . . . .22$

2. General procedure for the one-pot preparation of cis pyrrolidinones $1 \ldots . . . . . . . . .53$

3. General procedure for the one-step preparation of bispidines $2 \ldots \ldots \ldots \ldots \ldots \ldots . . . . . .55$

4. General procedure for the one-step preparation of bispidines 3.................... 6

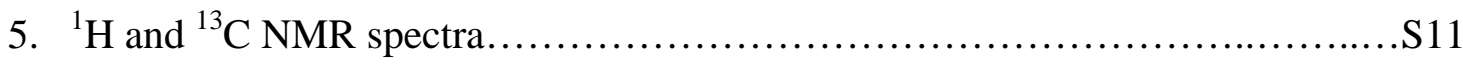


General. Unless otherwise noted, all reactions were conducted under $\mathrm{N}_{2}$ atmosphere using standard air-free manipulation techniques. Solvents were purchased from Fisher Scientific Company or Aldrich and used without further purification. Commercial reagents were purchased either from Aldrich and used without further purification. Chiral substrates 4 were prepared according to Deng's or Evan's procedures ${ }^{1}$.

High performance liquid chromatography (HPLC) analysis was performed using Agilent Technology 1100 series instrument with YMC Pack Pro C18 (240 x 4.6 mm I.D., $5 \mu \mathrm{m}$ particle size) column. Proton nuclear magnetic resonance ( ${ }^{1} \mathrm{H}$ NMR) spectra were measured on Bruker Avance-400 or 500 instrument ( 400 or $500 \mathrm{MHz}$ ). Carbon nuclear magnetic resonance $\left({ }^{13} \mathrm{C}\right.$ NMR) spectra were measured on Bruker Avance-400 or 500 instrument (100 or $125 \mathrm{MHz}$ ) with complete proton decoupling. Chemical shifts are reported in ppm downfield from tetramethylsilane (TMS).

\section{Procedure for the one-pot preparation of trans pyrrolidinones 1}

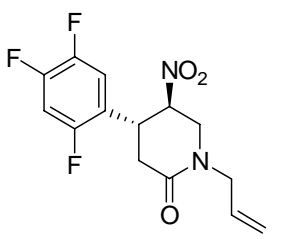

(4R,5R)-1-allyl-5-nitro-4-(2,4,5-trifluorophenyl)piperidin-2-one (1a): To a solution of nitro malonate $4 \mathrm{a}(100 \mathrm{~g}, 0.2983 \mathrm{~mol})$ and allyl amine (22.14 g, $0.3878 \mathrm{~mol})$ in iPrOH $(500 \mathrm{~mL})$ and water $(100 \mathrm{~mL})$ at $50{ }^{\circ} \mathrm{C}$ was added $37 \mathrm{wt} \%$ HCHO (25.42 g, $0.3132 \mathrm{~mol}$ ) dropwise over 1-2 h and the reaction solution was stirred for additional $2-3 \mathrm{~h}$ at $50{ }^{\circ} \mathrm{C} .5 \mathrm{~N}$ $\mathrm{NaOH}(104.4 \mathrm{~mL}, 0.522 \mathrm{~mol})$ was added in one portion at $50{ }^{\circ} \mathrm{C}$. The reaction solution was stirred additional $1-2 \mathrm{~h}$ at $50{ }^{\circ} \mathrm{C}$. Conc. $\mathrm{HCl}(54.2 \mathrm{~mL}, 0.6563 \mathrm{~mol})$ was added dropwise over $30 \mathrm{~min}$. between $50-60{ }^{\circ} \mathrm{C}$. The reaction solution was stirred at 55- $60{ }^{\circ} \mathrm{C}$ for $2-3 \mathrm{~h}$ and then cooled to $45{ }^{\circ} \mathrm{C}$ and $5 \% \mathrm{NaHCO}_{3}$ (ca. $100 \mathrm{~mL}$ ) was added dropwise to $\mathrm{pH}=7-8$. Then, water $(100 \mathrm{~mL})$ was added over $30 \mathrm{~min}$. The resulting slurry was stirred at $45{ }^{\circ} \mathrm{C}$ for additional $1-2 \mathrm{~h}$ and cooled to ambient temperature slowly. After aging overnight, the slurry was filtered. The wet cake was displacement washed with $50 \%$ aq. $\mathrm{iPrOH}(150 \mathrm{ml} \times 2$ ), and suction dried at ambient temperature to give $72 \mathrm{~g}$ of off-white to yellowish solid. $76 \%$ yield. The isolated crystalline trans pyrrolidinone $1 \mathbf{a}$ is stable at ambient temperature and no epimerization to its corresponding cis isomer was observed within a year.

1H-NMR (500 MHz, CDCl $)$ ): $\delta 7.04$ (m, $1 \mathrm{H}), 7.01$ (m, $1 \mathrm{H}), 5.76$ (m, $1 \mathrm{H}), 5.26$ (m, 2 H), 5.07 (m, $1 \mathrm{H}$ ), 4.13 (dd, $J=6.2,14.9 \mathrm{~Hz}, 1 \mathrm{H}$ ), 4.02 (m, $2 \mathrm{H}$ ), 3.91 (dd, $J=6.9,13.2$ $\mathrm{Hz}, 1 \mathrm{H}$ ), 3.72 (dd, $J=5.1,13.2 \mathrm{~Hz}, 1 \mathrm{H}$ ), 2.84 (dd, $J=6.2,17.5 \mathrm{~Hz}, 1 \mathrm{H}), 2.72$ (dd, $J=$ 9.8, $17.5 \mathrm{~Hz}, 1 \mathrm{H}$ ). 13C-NMR (125 MHz, $\mathrm{CDCl}_{3}$ ): $\delta 166.7,156.0$ (ddd, $J=2.5,10.0$, $245.0 \mathrm{~Hz}$ ), 150.1 (td, $J=13.6,251.3 \mathrm{~Hz}$ ), 147.3 (ddd, $J=3.8,12.5,246.3 \mathrm{~Hz}), 131.7$, 121.6 (td, $J=5.0,15.0 \mathrm{~Hz}$ ), 119.6, 117.1 (ddd, $J=1.3,6.3,20.0 \mathrm{~Hz}$ ), 106.9 (dd, $J=21.3$, $27.5 \mathrm{~Hz}$ ), 83.1 (d, $J=1.3 \mathrm{~Hz}$ ), 49.4, 47.7, 36.7, 35.0. Anal calc'd for $\mathrm{C}_{14} \mathrm{H}_{13} \mathrm{~F}_{3} \mathrm{~N}_{2} \mathrm{O}_{3}$ : C, 53.51; H, 4.17; N, 8.91; Found: C, 53.59; H, 4.12; N, 8.90.

\footnotetext{
${ }^{1}$ (a) Evans, D. A.; Seidel, D. J. Am. Chem. Soc. 2005, 127, 9958. (b) Li, H.; Wang, Y.; Tang, L.; Deng, L. J. Am. Chem. Soc. 2004, 127, 9906.
} 


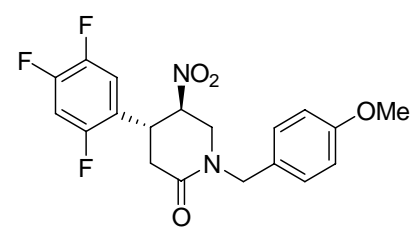

(4R,5R)-1-(4-methoxybenzyl)-5-nitro-4-(2,4,5-trifluorophenyl)piperidin-2-one: The title compound was prepared under similar conditions described above. ${ }^{1} \mathrm{H}-\mathrm{NMR}(400 \mathrm{MHz}$, $\left.\mathrm{CDCl}_{3}\right): \delta 7.19(\mathrm{~m}, 2 \mathrm{H}), 6.98(\mathrm{~m}, 1 \mathrm{H}), 6.86(\mathrm{~m}, 2 \mathrm{H}), 4.98$ (ddd, $J=5.2,6.9,8.3 \mathrm{~Hz}, 1 \mathrm{H}$ ), 4.76 (d, $J=14.4 \mathrm{~Hz}, 1 \mathrm{H}$ ), 4.37 (d, $J=14.4 \mathrm{~Hz}, 1 \mathrm{H}$ ), 3.98 (m, $1 \mathrm{H}$ ), 3.80 (dd, $J=6.5,13.1 \mathrm{~Hz}, 1 \mathrm{H}), 3.78$ (s, $3 \mathrm{H}), 3.58$ (dd, $J=5.1,13.1 \mathrm{~Hz}, 1 \mathrm{H}$ ), 2.85 (dd, $J=6.2,17.6 \mathrm{~Hz}, 1 \mathrm{H}$ ), 2.72 (dd, $J=9.4,17.6 \mathrm{~Hz}, 1$ H). 13C-NMR (100 MHz, CDCl $)$ ): $\delta$ 166.8, 159.6, 155.84 (ddd, $J=3.2,9.6,245.9 \mathrm{~Hz}$ ), 150.0 (ddd, $J=13.6,14.4,253.1 \mathrm{~Hz}$ ), 147.2 (ddd, $J=4.0,12.8,247.5 \mathrm{~Hz}$ ), 129.9, 127.7, 121.5 (td, $J=4.8,16.0 \mathrm{~Hz}$ ), 117.0 (dd, $J=5.6,20.0 \mathrm{~Hz}$ ), 114.4, 106.7 (dd, $J=20.8,28.0$ Hz) 82.8, 55.4, 49.7, 47.4, 37.3, 34.9. HRMS calc'd for $\mathrm{C}_{19} \mathrm{H}_{17} \mathrm{~F}_{3} \mathrm{~N}_{2} \mathrm{O}_{3}[\mathrm{M}+\mathrm{H}]^{+}$395.1213, found 395.1221 .

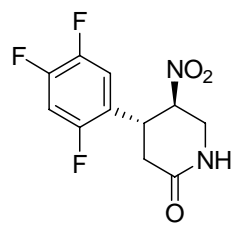

(4R,5R)-5-nitro-4-(2,4,5-trifluorophenyl)piperidin-2-one: The title compound and its cis isomer were prepared by deprotection of $\mathbf{1 a}$ in the presence of catalytic amount of $\mathrm{RhCl}_{3}$ in $\mathrm{nPrOH}$ at $95{ }^{\circ} \mathrm{C}$ followed by aqueous $\mathrm{HCl}$ treatment as shown in Scheme S1. The crystalline trans product could be prepared under similar isomerization conditions described above. ${ }^{1} \mathrm{H}-\mathrm{NMR}\left(400 \mathrm{MHz}, d_{4}-\mathrm{MeOH}\right.$ ): $\delta 7.38(\mathrm{~m}, 1 \mathrm{H}), 7.20(\mathrm{~m}, 1 \mathrm{H}), 5.31$ (m, $1 \mathrm{H}$ ), 4.11 (m, $1 \mathrm{H}), 4.02$ (m, $2 \mathrm{H}$ ), 3.86 (dd, $J=7.7,12.9 \mathrm{~Hz}, 1 \mathrm{H}$ ), 2.70 (dd, $J=7.2$, $17.7 \mathrm{~Hz}, 1 \mathrm{H}$ ), 2.64 (dd, $J=9.6,17.7 \mathrm{~Hz}, 1 \mathrm{H}$ ). 13C-NMR (100 MHz, $\left.d_{4}-\mathrm{MeOH}\right): \delta 172.3$, 157.5 (ddd, $J=2.4,9.6,244.9 \mathrm{~Hz}$ ), 151.1 (ddd, $J=12.8,14.5,250.6 \mathrm{~Hz}$ ), 148.5 (ddd, $J=$ 3.2, 12.0, $244.9 \mathrm{~Hz}$ ), 124.2 (td, $J=4.8,16.1 \mathrm{~Hz}$ ), 118.2 (dd, $J=5.6,21.7 \mathrm{~Hz}$ ), 107.4 (dd, $J$ $=20.9,28.9 \mathrm{~Hz}$ ), 84.2, 44.5, 37.8, 36.0. HRMS calc'd for $\mathrm{C}_{11} \mathrm{H}_{8} \mathrm{~F}_{3} \mathrm{~N}_{2} \mathrm{O}_{3}[\mathrm{M}+\mathrm{H}]^{+}$ 275.0638, found 275.0641 .

Scheme S1

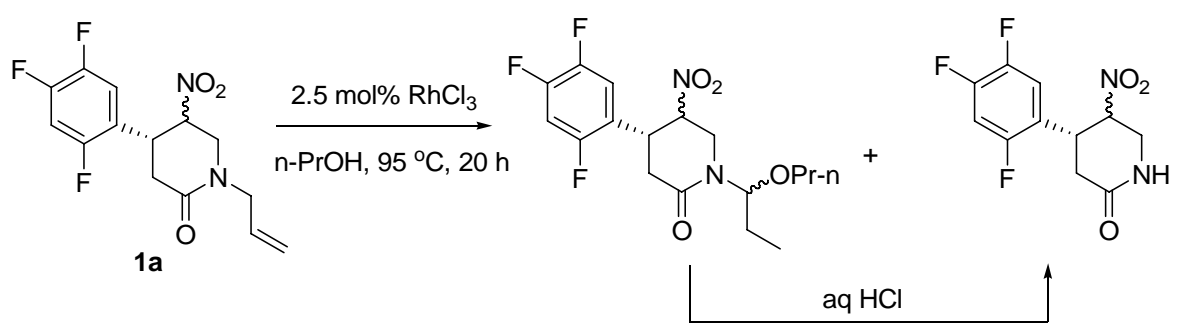

\section{General procedure for the one-pot preparation of cis pyrrolidinones 1}

To a solution of nitro malonate 4 (3.0 mmol) and allyl amine $(223 \mathrm{mg}, 3.9 \mathrm{mmol})$ in iPrOH $(5 \mathrm{~mL})$ and water $(1 \mathrm{~mL})$ at $50-55{ }^{\circ} \mathrm{C}$ was added a solution of $37 \mathrm{wt} \% \mathrm{HCHO}(256$ $\mathrm{mg}, 3.15 \mathrm{mmol})$ in water $(1 \mathrm{~mL})$ dropwise over $1.5 \mathrm{~h}$ and the reaction solution was stirred for additional $3 \mathrm{~h}$ at $55^{\circ} \mathrm{C} .5 \mathrm{~N} \mathrm{NaOH}(0.9 \mathrm{~mL}, 4.5 \mathrm{mmol})$ was added in one portion at 55 ${ }^{\circ} \mathrm{C}$. The reaction solution was stirred additional $2 \mathrm{~h}$ at $55{ }^{\circ} \mathrm{C}$. Conc. $\mathrm{HCl}(1.25 \mathrm{~mL}, 15$ mmol) was added dropwise between $50-60{ }^{\circ} \mathrm{C}$. The reaction solution was stirred at 55- 
$60{ }^{\circ} \mathrm{C}$ for $3-5 \mathrm{~h}$ and then cooled to ambient temperature. EtOAc $(80 \mathrm{~mL})$ was added and the oragic pahse was washed with water $(10 \mathrm{~mL})$ followed by brine $(10 \mathrm{~mL})$. Upon concentration, the residue was purified on silica gel column eluting with EtOAc/hexane to afford the desired cis pyrrolidinone $\mathbf{1}$.

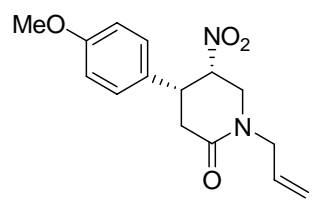

(4R,5S)-1-allyl-4-(4-methoxyphenyl)-5-nitropiperidin-2-one: 1 $_{\mathrm{H}-}$ NMR (400 MHz, $\left.\mathrm{CDCl}_{3}\right): \delta 7.04(\mathrm{~d}, J=6.8 \mathrm{~Hz}, 2 \mathrm{H}), 6.88(\mathrm{~d}, J=6.8$ $\mathrm{Hz}, 2 \mathrm{H}$ ), 5.80 (m, $1 \mathrm{H}), 5.28$ (s, $1 \mathrm{H}), 5.25$ (dd, $J=1.1,6.3 \mathrm{~Hz}, 1 \mathrm{H}$ ), 5.02 (dd, $J=5.2,9.9 \mathrm{~Hz}, 1 \mathrm{H}), 4.17$ (dd, $J=6.1,15.0 \mathrm{~Hz}, 1 \mathrm{H}), 4.04$ (dd, $J=6.1,15.0 \mathrm{~Hz}, 1 \mathrm{H}$ ), 3.84 (m, $1 \mathrm{H}$ ), 3.80 (s, $3 \mathrm{H}$ ), 3.73 (dd, $J=6.3,13.5 \mathrm{~Hz}, 1 \mathrm{H}$ ), 3.66 (dd, $J=5.1,13.5 \mathrm{~Hz}, 1 \mathrm{H}$ ), 3.03 (dd, $J=7.5,17.9 \mathrm{~Hz}, 1 \mathrm{H}$ ), 2.90 (dd, $J=6.4,17.9$ $\mathrm{Hz}, 1 \mathrm{H}) .13 \mathrm{C}-\mathrm{NMR}\left(100 \mathrm{MHz}, \mathrm{CDCl}_{3}\right): \delta 167.6,159.8,131.80,128.6,128.2,119.2$, 114.7, 82.5, 55.5, 49.5, 46.1, 40.6, 34.4. HRMS calc'd for $\mathrm{C}_{15} \mathrm{H}_{18} \mathrm{~N}_{2} \mathrm{O}_{4}[\mathrm{M}+\mathrm{H}]^{+}$291.1339, found 291.1333.

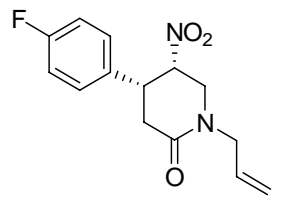

(4R,5S)-1-allyl-4-(4-fluorophenyl)-5-nitropiperidin-2-one: 1 H-NMR (500 MHz, $\left.\mathrm{CDCl}_{3}\right): \delta 7.11(\mathrm{~m}, 2 \mathrm{H}), 7.05(\mathrm{~m}, 2 \mathrm{H}), 5.78(\mathrm{~m}, 1 \mathrm{H}), 5.28$ (m, $1 \mathrm{H}), 5.26$ (m, $1 \mathrm{H}), 5.04(\mathrm{~m}, 1 \mathrm{H}), 4.18$ (dd, $J=6.1,15.0 \mathrm{~Hz}, 1 \mathrm{H})$, 4.03 (dd, $J=6.4,15.0 \mathrm{~Hz}, 1 \mathrm{H}$ ), 3.87 (m, $1 \mathrm{H}$ ), 3.74 (dd, $J=6.0,13.6$ $\mathrm{Hz}, 1 \mathrm{H}$ ), 3.69 (dd, $J=5.3,13.6 \mathrm{~Hz}, 1 \mathrm{H}$ ), 3.02 (dd, $J=7.9,17.9 \mathrm{~Hz}, 1 \mathrm{H}$ ), 2.91 (dd, $J=$ 6.4, $17.9 \mathrm{~Hz}, 1 \mathrm{H})$. 13C-NMR (125 MHz, $\left.\mathrm{CDCl}_{3}\right): \delta$ 167.2, 162.8 (d, $\left.J=246.3 \mathrm{~Hz}\right), 132.1$ (d, $J=2.5 \mathrm{~Hz}), 131.7,129.2$ (d, $J=7.5 \mathrm{~Hz}), 119.3,116.3(\mathrm{~d}, J=21.3 \mathrm{~Hz}), 82.3,49.5$, 46.2, 40.6, 34.1. HRMS calc'd for $\mathrm{C}_{14} \mathrm{H}_{16} \mathrm{FN}_{2} \mathrm{O}_{3}[\mathrm{M}+\mathrm{H}]^{+}$279.1140, found 279.1149.

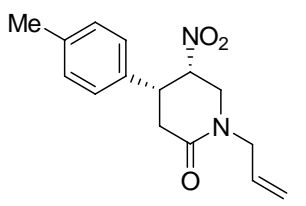

(4R,5S)-1-allyl-4-(4-methylphenyl)-5-nitropiperidin-2-one: $\quad 1_{\mathrm{H}-}$ NMR (500 MHz, $\left.\mathrm{CDCl}_{3}\right): \delta 7.16(\mathrm{~d}, J=8.0,2 \mathrm{H}), 7.01(\mathrm{~d}, J=8.0 \mathrm{~Hz}, 2$ H), 5.80 (m, 1 H), 5.28 (s, 1 H), 5.25 (m 1 H), 5.03 (m, 1 H), 4.18 (dd, $J$ = 6.1, $15.0 \mathrm{~Hz}, 1 \mathrm{H}), 4.04$ (dd, $J=6.3,15.0 \mathrm{~Hz}, 1 \mathrm{H}), 3.85(\mathrm{~m}, 1 \mathrm{H})$, 3.74 (dd, $J=6.3 \mathrm{~Hz}, 13.5 \mathrm{~Hz}, 1 \mathrm{H}$ ), 3.66 (dd, $J=5.1,13.5 \mathrm{~Hz}, 1 \mathrm{H}$ ), 3.05 (dd, $J=7.6,17.9 \mathrm{~Hz}, 1 \mathrm{H}$ ), 2.91 (dd, $J=6.4,17.9 \mathrm{~Hz}, 1 \mathrm{H}$ ), 2.34 (s, $3 \mathrm{H}$ ). 13C-NMR (125 MHz, $\left.\mathrm{CDCl}_{3}\right): \delta 167.6,138.6,133.2,131.8,130.0,127.4,119.2,82.4,49.5,46.2$, 41.0, 34.2, 21.3. HRMS calc'd for $\mathrm{C}_{15} \mathrm{H}_{19} \mathrm{~N}_{2} \mathrm{O}_{3}[\mathrm{M}+\mathrm{H}]^{+}$275.1390, found 275.1385.

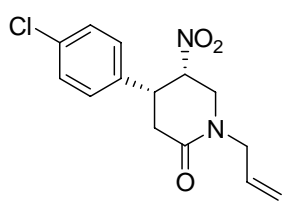

(4R,5S)-1-allyl-4-(4-chlorophenyl)-5-nitropiperidin-2-one: 1H-NMR (500 MHz, $\left.\mathrm{CDCl}_{3}\right): \delta 7.32$ (d, $\left.J=8.5 \mathrm{~Hz}, 2 \mathrm{H}\right), 7.07$ (d, $J=8.5 \mathrm{~Hz}, 2$ $\mathrm{H}), 5.79$ (m, $1 \mathrm{H}), 5.28$ (m, $1 \mathrm{H}), 2.25$ (m, $1 \mathrm{H}), 5.04$ (m, $1 \mathrm{H}), 4.18$ (dd, $J=6.1,14.9 \mathrm{~Hz}, 1 \mathrm{H}), 4.04$ (dd, $J=6.3,14.9 \mathrm{~Hz}, 1 \mathrm{H}), 3.86(\mathrm{~m}, 1 \mathrm{H})$, 3.74 (dd, $J=6.1,13.6 \mathrm{~Hz}, 1 \mathrm{H}$ ), 3.69 (dd, $J=5.2,13.6 \mathrm{~Hz}, 1 \mathrm{H}$ ), 3.02 (dd, $J=7.8,17.8 \mathrm{~Hz}, 1 \mathrm{H}), 2.91$ (dd, $J=6.3,17.8 \mathrm{~Hz}, 1 \mathrm{H}$ ). 13C-NMR (125 MHz, $\left.\mathrm{CDCl}_{3}\right): \delta 167.1,134.8,131.7,129.6,128.9,119.4,82.1,49.5,46.2,40.7,34.0$. HRMS calc'd for $\mathrm{C}_{14} \mathrm{H}_{16} \mathrm{ClN}_{2} \mathrm{O}_{3}[\mathrm{M}+\mathrm{H}]^{+}$295.0844, found 295.0842 . 


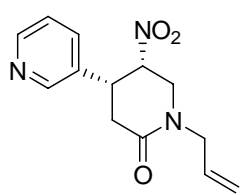

(4R,5S)-1-allyl-5-nitro-4-pyridin-3-ylpiperidin-2-one: $1 \mathrm{H}-\mathrm{NMR}$ (500 $\left.\mathrm{MHz}, \mathrm{CDCl}_{3}\right): \delta 8.52(\mathrm{~m}, 2 \mathrm{H}), 7.55(\mathrm{~m}, 1 \mathrm{H}), 7.28(\mathrm{~m}, 1 \mathrm{H}), 5.70(\mathrm{~m}, 1 \mathrm{H})$, 5.21 (m, 2 H), 5.01 (m, $1 \mathrm{H}), 4.07$ (m, $1 \mathrm{H}), 3.99$ (dd, $J=6.3,14.9$, Hz, 1 H), 3.86 (m, 2 H), 3.67 (dd, $J=5.2,13.1 \mathrm{~Hz}, 1 \mathrm{H}$ ), 2.82 (dd, $J=6.1,17.7$

$\mathrm{Hz}, 1 \mathrm{H}$ ), 2.68 (dd, $J=9.6,17.7 \mathrm{~Hz}, 1 \mathrm{H}$ ). 13C-NMR (125 MHz, $\mathrm{CDCl}_{3}$ ): $\delta$ 166.6, 149.7, 148.9, 134.6, 133.8, 131.5, 124.1, 119.3, 84.4, 49.2, 47.5, 40.3, 35.8. HRMS calc'd for $\mathrm{C}_{13} \mathrm{H}_{16} \mathrm{~N}_{3} \mathrm{O}_{3}[\mathrm{M}+\mathrm{H}]^{+}$262.1186, found 262.1190.

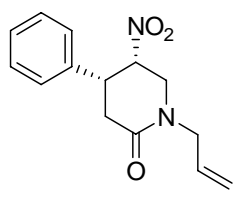

(4R,5S)-1-allyl-5-nitro-4-phenylpiperidin-2-one: 1 $\mathrm{H}-\mathrm{NMR}$ (500 $\mathrm{MHz}$, $\left.\mathrm{CDCl}_{3}\right): \delta 7.35$ (m, $\left.3 \mathrm{H}\right), 7.13(\mathrm{~m}, 2 \mathrm{H}), 5.79(\mathrm{~m}, 1 \mathrm{H}), 5.28(\mathrm{~s}, 1 \mathrm{H}), 5.26$ (m, $1 \mathrm{H}), 5.07$ (m, $1 \mathrm{H}), 4.19$ (dd, $J=6.1,15.0 \mathrm{~Hz}, 1 \mathrm{H}), 4.05$ (dd, $J=6.3$, $15.0 \mathrm{~Hz}, 1 \mathrm{H}$ ), 3.88 (m, $1 \mathrm{H}$ ), 3.75 (dd, $J=6.1,13.5 \mathrm{~Hz}, 1 \mathrm{H}$ ), 3.69 (dd, $J=$ 5.1, 13.5 Hz, 1 H), 3.08 (dd, $J=7.8,17.9$ Hz, 1 H), 2.93 (dd, $J=6.4,17.9$

$\mathrm{Hz}, 1 \mathrm{H})$. 13C-NMR (125 MHz, $\left.\mathrm{CDCl}_{3}\right): \delta$ 167.3, 136.1, 131.6, 129.1, 128.5, 127.3, 119.0, 82.2, 49.3, 46.1, 41.0, 33.8. HRMS calc'd for $\mathrm{C}_{14} \mathrm{H}_{17} \mathrm{~N}_{2} \mathrm{O}_{3}[\mathrm{M}+\mathrm{H}]^{+}$261.1234, found 262.1235 .

\section{General procedure for the one-step preparation of bispidines 2}

To a solution of nitro malonate $4(1.5 \mathrm{mmol})$ in i-PrOH $(7 \mathrm{~mL})$ and water $(1.5 \mathrm{~mL})$ at ambient temperature was added t- $\mathrm{BuCO}_{2} \mathrm{H}(77 \mathrm{mg}, 0.75 \mathrm{mmol})$ followed by ally amine (214 mg, $3.75 \mathrm{mmol}$ ). Then, the reaction solution was heated to $55{ }^{\circ} \mathrm{C}$ and a solution of 37 wt\% HCHO (268 mg, $3.3 \mathrm{mmol})$ in water $(1 \mathrm{~mL})$ was added dropwise over $2.5 \mathrm{~h}$. After addition, the reaction solution was agitated at $55{ }^{\circ} \mathrm{C}$ for $5-10 \mathrm{~h}$. EtOAc $(80 \mathrm{~mL})$ was added and the organic phase was washed with water $(10 \mathrm{~mL})$ followed by brine $(10 \mathrm{~mL})$. Upon concentration, the residue was purified on silica gel column eluting with EtOAc/hexane to afford the desired 2.

\section{General procedure for the one-step preparation of bispidines 3}

To a solution of nitro malonate $4(1.5 \mathrm{mmol})$ in i-PrOH $(7 \mathrm{~mL})$ and water $(1.5 \mathrm{~mL})$ at ambient temperature was added $\mathrm{MeSO}_{3} \mathrm{H}(72 \mathrm{mg}, 0.75 \mathrm{mmol})$ followed by ally amine (214 mg, $3.75 \mathrm{mmol}$ ). Then, the reaction solution was heated to $55{ }^{\circ} \mathrm{C}$ and a solution of 37 wt\% HCHO (487 mg, $6.0 \mathrm{mmol})$ in water $(1 \mathrm{~mL})$ was added dropwise over $2.5 \mathrm{~h}$. After addition, the reaction solution was agitated at $55{ }^{\circ} \mathrm{C}$ for $5-10 \mathrm{~h}$. EtOAc $(80 \mathrm{~mL})$ was added and the organic phase was washed with water $(10 \mathrm{~mL})$ followed by brine $(10 \mathrm{~mL})$. Upon concentration, the residue was purified on silica gel column eluting with EtOAc/hexane to afford the desired 3.

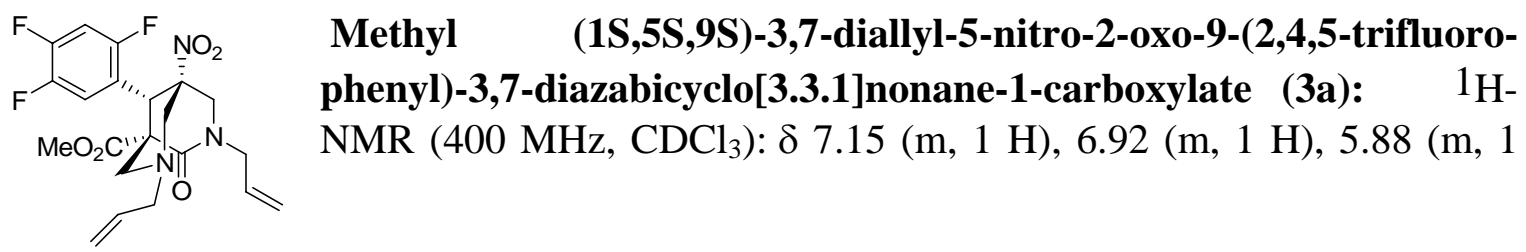


H), 5.72 (m, $1 \mathrm{H}$ ), 5.45 (dd, $J=0.7,17.2 \mathrm{~Hz}, 1 \mathrm{H}), 5.39$ (d, $J=10.1 \mathrm{~Hz}, 1 \mathrm{H}), 5.23$ (m, 2 H), 4.40 (dd, $J=5.9,14.7 \mathrm{~Hz}, 1 \mathrm{H}$ ), 4.29 (s, $1 \mathrm{H}$ ), 4.17 (dd, $J=1.6,13.3 \mathrm{~Hz}, 1 \mathrm{H}$ ), 3.99 (dd, $J=6.8,14.7 \mathrm{~Hz}, 1 \mathrm{H}), 3.77(\mathrm{~d}, J=13.3 \mathrm{~Hz}, 1 \mathrm{H}), 3.53(\mathrm{dd}, J=1.3,11.6 \mathrm{~Hz}, 1 \mathrm{H}$ ), 3.46 (s, $3 \mathrm{H}$ ), 3.37 (dd, $J=1.0,10.4 \mathrm{~Hz}, 1 \mathrm{H}$ ), 3.24 (dd, $J=6.0,13.6 \mathrm{~Hz}, 1 \mathrm{H}$ ), 3.10 (dd, $J$ = 6.9, $13.6 \mathrm{~Hz}, 1 \mathrm{H}), 2.94$ (dd, $J=1.2,10.3 \mathrm{~Hz}, 1 \mathrm{H}), 2.86$ (d, $J=11.5 \mathrm{~Hz}, 1 \mathrm{H}) .13 \mathrm{C}-$ NMR (100 MHz, $\mathrm{CDCl}_{3}$ ): $\delta$ 168.1, 166.3, 156.8 (ddd, $J=3.2,8.8,248.2 \mathrm{~Hz}$ ), 150.0 (td, $J$ = 13.7, $253.8 \mathrm{~Hz}$ ), 146.9 (ddd, $J=3.2,12.0,245.7 \mathrm{~Hz}$ ), 133.0, 130.8, 120.5, 120.1, 118.0 (td, $J=5.3,13.7 \mathrm{~Hz}$ ), $117.4(\mathrm{~m}), 106.0$ (dd, $J=20.0,30.0 \mathrm{~Hz}$ ), 84.2, 65.7, 60.4, 60.0, 56.5, 52.7, 50.1, 49.9, 43.6. HRMS calc'd for $\mathrm{C}_{21} \mathrm{H}_{23} \mathrm{~F}_{3} \mathrm{~N}_{3} \mathrm{O}_{5}[\mathrm{M}+\mathrm{H}]^{+}$454.1584, found 454.1594 .

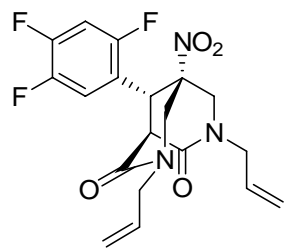

(1R,5R,9R)-3,7-diallyl-5-nitro-9-(2,4,5-trifluorophenyl)-3,7-diazabicyclo[3.3.1]nonane-2,8-dione (2a): $1 \mathrm{H}-\mathrm{NMR}\left(500 \mathrm{MHz}, \mathrm{CDCl}_{3}\right): \delta$ 6.99 (m, $1 \mathrm{H}), 6.85$ (m, $1 \mathrm{H}), 5.80$ (m, $2 \mathrm{H}), 5.39$ (d, $J=7.9 \mathrm{~Hz}, 1 \mathrm{H})$, 5.36 (dd, $J=1.1,8.0 \mathrm{~Hz}, 1 \mathrm{H}), 5.33(\mathrm{~s}, 1 \mathrm{H}), 5.28$ (dd, $J=1.2,17.1$ Hz, $1 \mathrm{H}$ ), 4.45 (s, $1 \mathrm{H}$ ), 4.22 (dd, $J=1.1,12.8 \mathrm{~Hz}, 1 \mathrm{H}$ ), 4.15 (m, $4 \mathrm{H}$ ), 4.05 (dd, $J=6.4,15.0 \mathrm{~Hz}, 1 \mathrm{H}$ ), 3.71 (d $J=2.3 \mathrm{~Hz}, 1 \mathrm{H}), 3.70(\mathrm{~s}, 1 \mathrm{H}$ ), 3.68 (s, $1 \mathrm{H}$ ).13C-NMR (125 MHz, $\mathrm{CDCl}_{3}$ ): $\delta 163.7,162.3,156.4$ (ddd, $J=2.8,9.2$, $248.6 \mathrm{~Hz}$ ), 150.5 (td, $J=14.2,255.3 \mathrm{~Hz}$ ), 147.4 (ddd, $J=3.7,12.6,247.3 \mathrm{~Hz}$ ), 131.2, 130.6, 121.5, 120.2, 117.5 (td, $J=4.9,15.1 \mathrm{~Hz}$ ), 116.4 (ddd, $J=1.5,4.3,20.3 \mathrm{~Hz}), 107.0$ (dd, $J=17.0,24.0 \mathrm{~Hz}$ ), 84.2, 56.9, 52.8, 50.6, 50.1, 49.1, 39.3. HRMS calc'd for $\mathrm{C}_{19} \mathrm{H}_{19} \mathrm{~F}_{3} \mathrm{~N}_{3} \mathrm{O}_{4}[\mathrm{M}+\mathrm{H}]^{+}$410.1322, found 410.1311. Anal calc'd for $\mathrm{C}_{19} \mathrm{H}_{18} \mathrm{~F}_{3} \mathrm{~N}_{3} \mathrm{O}_{4}$ : C, 55.75; H, 4.43; N, 10.26; Found: C, 55.67; H, 4.41; N, 10.22.

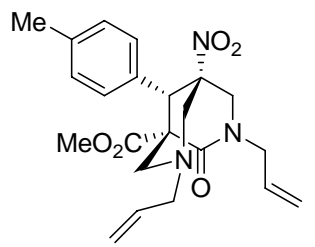

Methyl (1S,5S,9S)-3,7-diallyl-9-(4-methylphenyl)-5-nitro-2-oxo3,7-diazabicyclo[3.3.1]nonane-1-carboxylate: 1H-NMR (500 MHz, $\mathrm{CDCl}_{3}$ ): $\delta 7.08$ (d, $\left.J=8.4 \mathrm{~Hz}, 2 \mathrm{H}\right), 7.06$ (d, $\left.J=8.4 \mathrm{~Hz}, 2 \mathrm{H}\right), 5.89$ (m, $1 \mathrm{H}), 5.73(\mathrm{~m}, 1 \mathrm{H}), 5.43(\mathrm{~m}, 1 \mathrm{H}), 5.33(\mathrm{~m}, 1 \mathrm{H}), 5.23(\mathrm{~m}, 1 \mathrm{H}), 5.20$ (m, $1 \mathrm{H}), 4.56$ (m, $1 \mathrm{H}), 4.13$ (dd, $J=2.1,12.9 \mathrm{~Hz}, 1 \mathrm{H}), 3.84$ (dd, $J=$ 7.0, $14.8 \mathrm{~Hz}, 1 \mathrm{H}$ ), 3.78 (s, $1 \mathrm{H}$ ), 3.64 (dd, $J=0.9,12.9 \mathrm{~Hz}, 1 \mathrm{H}$ ), 3.47 (dd, $J=1.6,11.2$ Hz, 1 H), 3.33 (s, 3 H), 3.32 (dd, $J=1.6,11.2 \mathrm{~Hz}, 1 \mathrm{H}$ ), 3.21 (dd, $J=1.3$, 6.0, $13.5 \mathrm{~Hz}, 1$ H), 3.06 (dd, $J=6.9,13.5 \mathrm{~Hz}, 1 \mathrm{H}$ ), 2.99 (dd, $J=2.0,10.3 \mathrm{~Hz}, 1 \mathrm{H}$ ), 2.86 (d, $J=11.2 \mathrm{~Hz}$, $1 \mathrm{H}), 2.29$ (s, $3 \mathrm{H}$ ). 13C-NMR (125 MHz, $\left.\mathrm{CDCl}_{3}\right): \delta$ 168.9, 166.6, 138.5, 133.3, 131.4, 129.7, 129.6, 129.3, 119.39, 119.37, 85.1, 65.1, 60.9, 59.9, 56.5, 54.1, 52.1, 49.6, 49.4, 21.0. HRMS calc'd for $\mathrm{C}_{22} \mathrm{H}_{28} \mathrm{~N}_{3} \mathrm{O}_{5}[\mathrm{M}+\mathrm{H}]^{+}$414.2024, found 414.2012.

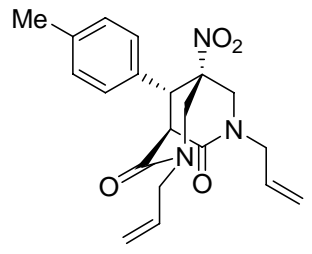

(1R,5R,9R)-3,7-diallyl-9-(4-methylphenyl)-5-nitro-3,7-diazabicyclo[3.3.1]nonane-2,8-dione: $1 \mathrm{H}-\mathrm{NMR}\left(500 \mathrm{MHz}, \mathrm{CDCl}_{3}\right): \delta$ 7.14 (d, $J=8.1 \mathrm{~Hz}, 2 \mathrm{H}), 6.93$ (d, $J=8.2 \mathrm{~Hz}, 2 \mathrm{H}), 5.76$ (m, $1 \mathrm{H})$, 5.29 (m, $2 \mathrm{H}), 4.27$ (dd, $J=6.2,14.6 \mathrm{~Hz}, 1 \mathrm{H}), 4.15$ (m, $3 \mathrm{H}), 4.06$ (dd, $J=6.2,15.1 \mathrm{~Hz}, 1 \mathrm{H}$ ), 3.96 (dd, $J=7.0,14.6 \mathrm{~Hz}, 1 \mathrm{H}$ ), 3.82 (d, $J$ = $13.1 \mathrm{~Hz}, 1 \mathrm{H}), 3.81(\mathrm{~d}, J=2.0 \mathrm{~Hz}, 1 \mathrm{H}), 3.68(\mathrm{~d}, J=13.1 \mathrm{~Hz}, 1 \mathrm{H})$, 
3.52 (dd, $J=1.7,12.5 \mathrm{~Hz}, 1 \mathrm{H}$ ), 2.33 (s, $3 \mathrm{H}$ ).13C-NMR (125 MHz, CDCl $)$ ): $\delta$ 164.7, 163.3, 139.2, 131.2, 131.0, 130.13, 130.09, 127.3, 120.5, 119.5, 85.4, 55.9, 52.7, 49.88, 49.87, 48.6, 45.4, 21.0. HRMS calc'd for $\mathrm{C}_{20} \mathrm{H}_{24} \mathrm{~N}_{3} \mathrm{O}_{4}[\mathrm{M}+\mathrm{H}]^{+}$370.1761, found 370.1759. Anal calc'd for $\mathrm{C}_{20} \mathrm{H}_{23} \mathrm{~N}_{3} \mathrm{O}_{4}$ : C, 65.03; H, 6.28; N, 11.37; Found: C, 64.97; H, 6.32; N, 11.39 .

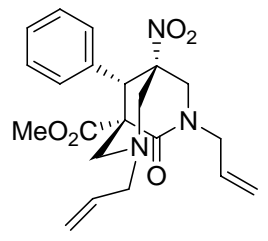

Methyl (1S,5S,9S)-3,7-diallyl-9-phenyl-5-nitro-2-oxo-3,7-diazabicyclo[3.3.1]nonane-1-carboxylate: $1 \mathrm{H}-\mathrm{NMR}\left(500 \mathrm{MHz}, \mathrm{CDCl}_{3}\right): \delta$ 7.27 (m, 3 H), 7.21 (m, 2 H), 5.89 (m, 1 H), 5.74 (m, 1 H), 5.43 (dd, $J=$

1.3, 17.2 Hz, $1 \mathrm{H}$ ), 5.34 (dd, $J=0.9,10.3 \mathrm{~Hz}, 1 \mathrm{H}$ ), 5.24 (m, $1 \mathrm{H}$ ), 5.21 (s, $1 \mathrm{H}), 4.58(\mathrm{~m}, 1 \mathrm{H}), 4.15$ (dd, $J=2.1,13.0 \mathrm{~Hz}, 1 \mathrm{H}), 3.85$ (dd, $J=6.9$, $14.9 \mathrm{~Hz}, 1 \mathrm{H}), 3.81(\mathrm{~s}, 1 \mathrm{H}), 3.67(\mathrm{~d}, J=13.0 \mathrm{~Hz}, 1 \mathrm{H}), 3.48$ (dd, $J=1.5,11.2 \mathrm{~Hz}, 1 \mathrm{H}$ ), 3.34 (dd, $J=1.4,10.3 \mathrm{~Hz}, 1 \mathrm{H}$ ), 3.30 (s, $1 \mathrm{H}$ ), 3.22 (dd, $J=6.0,13.5 \mathrm{~Hz}, 1 \mathrm{H}$ ), 3.07 (dd, $J$ = 6.9, $13.5 \mathrm{~Hz}, 1 \mathrm{H}), 2.99$ (dd, $J=2.0,10.3 \mathrm{~Hz}, 1 \mathrm{H}), 2.88$ (d, $J=11.2 \mathrm{~Hz}, 1 \mathrm{H}) .13 \mathrm{C}-$ NMR (125 MHz, $\mathrm{CDCl}_{3}$ ): $\delta$ 169.1, 166.7, 133.4, 133.1, 131.5, 130.0, 128.9, 128.8, 119.7, 85.2, 65.4, 61.1, 60.1, 56.8, 54.7, 52.3, 49.8, 49.6. HRMS calc'd for $\mathrm{C}_{21} \mathrm{H}_{26} \mathrm{~N}_{3} \mathrm{O}_{5}[\mathrm{M}+\mathrm{H}]^{+}$ 400.1867, found 400.1865. Anal calc'd for $\mathrm{C}_{21} \mathrm{H}_{25} \mathrm{~N}_{3} \mathrm{O}_{5}$ : C, 63.14; H, 6.31; N, 10.52; Found: C, 62.79; H, 6.30; N, 10.38 .

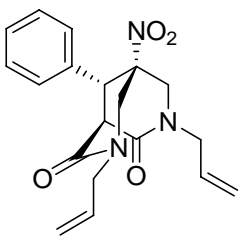

(1R,5R,9R)-3,7-diallyl-5-nitro-9-phenyl-3,7-diazabicyclo[3.3.1]nonane-2,8-dione: $1 \mathrm{H}-\mathrm{NMR}\left(500 \mathrm{MHz}, \mathrm{CDCl}_{3}\right): \delta 7.35$ (m, $\left.3 \mathrm{H}\right), 7.06$ (m, $2 \mathrm{H}), 5.79$ (m, $2 \mathrm{H}), 5.30$ (m, $4 \mathrm{H}), 4.30$ (m, $1 \mathrm{H}), 4.18$ (m, $2 \mathrm{H}), 4.14$ (dd, $J=6.1,15.1 \mathrm{~Hz}, 1 \mathrm{H}$ ), 4.07 (dd, $J=6.1,15.1 \mathrm{~Hz}, 1 \mathrm{H}$ ), 3.95 (dd, $J=$ 7.0, $14.6 \mathrm{~Hz}, 1 \mathrm{H}), 3.84$ (d, $J=2.0 \mathrm{~Hz}, 1 \mathrm{H}), 3.82(\mathrm{~d}, J=13.1 \mathrm{~Hz}, 1 \mathrm{H})$, 3.69 (d, $J=13.1 \mathrm{~Hz}, 1 \mathrm{H}), 3.54$ (dd, $J=1.8,12.6 \mathrm{~Hz}, 1 \mathrm{H}) .13 \mathrm{C}-\mathrm{NMR}\left(125 \mathrm{MHz}, \mathrm{CDCl}_{3}\right)$ : $\delta$ 164.8, 163.4, 133.4, 131.4, 131.1, 129.7, 129.5, 127.8, 120.8, 119.8, 85.5, 56.2, 52.8, 50.09, 50.07, 48.8, 45.9. HRMS calc'd for $\mathrm{C}_{19} \mathrm{H}_{22} \mathrm{ClN}_{3} \mathrm{O}_{4}[\mathrm{M}+\mathrm{H}]^{+}$356.1605, found 356.1596.

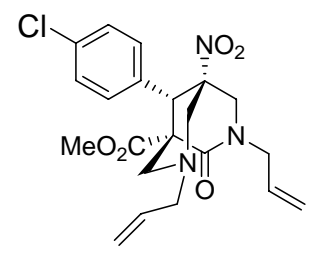

Methyl (1S,5S,9S)-3,7-diallyl-9-(4-chlorophenyl)-5-nitro-2-oxo3,7-diazabicyclo[3.3.1]nonane-1-carboxylate: $1 \mathrm{H}-\mathrm{NMR}(500 \mathrm{MHz}$, $\left.\mathrm{CDCl}_{3}\right): \delta 7.25$ (m, $\left.2 \mathrm{H}\right), 7.16(\mathrm{~m}, 2 \mathrm{H}), 5.88$ (m, $\left.1 \mathrm{H}\right), 5.73(\mathrm{~m}, 1 \mathrm{H})$, 5.43 (m, $1 \mathrm{H}), 5.34$ (dd, $J=1.1,10.2 \mathrm{~Hz}, 1 \mathrm{H}), 5.21(\mathrm{~m}, 2 \mathrm{H}), 4.53$ (m, $1 \mathrm{H}), 4.07$ (dd, $J=2.1,13.1 \mathrm{~Hz}, 1 \mathrm{H}), 3.85(6.9,14.8 \mathrm{~Hz}, 1 \mathrm{H})$, $3.80(\mathrm{~s}, 1 \mathrm{H}), 3.67$ (d, $J=13.1 \mathrm{~Hz}, 1 \mathrm{H}), 3.49$ (dd, $J=1.5,11.3 \mathrm{~Hz}, 1 \mathrm{H}), 3.37$ (s, $3 \mathrm{H}$ ), 3.34 (dd, $J=1.5 \mathrm{~Hz}, 10.3 \mathrm{~Hz}, 1 \mathrm{H}$ ), $3.21(\mathrm{~m}, 1 \mathrm{H}$ ), 3.07 (dd, $J=7.0,13.5 \mathrm{~Hz}, 1 \mathrm{H}$ ), 2.97 (dd, $J=2.0,10.3 \mathrm{~Hz}, 1 \mathrm{H}), 2.85$ (d, $J=11.3 \mathrm{~Hz}, 1 \mathrm{H}) .13 \mathrm{C}-\mathrm{NMR}\left(125 \mathrm{MHz}, \mathrm{CDCl}_{3}\right): \delta$ 168.8, 166.5, 135.0, 133.3, 131.2, 131.4, 131.3, 129.0, 119.9, 119.8, 85.1, 65.3, 61.0, 60.1, 56.6, 53.9, 52.5, 49.9, 49.5. HRMS calc'd for $\mathrm{C}_{21} \mathrm{H}_{25} \mathrm{ClN}_{3} \mathrm{O}_{5}[\mathrm{M}+\mathrm{H}]^{+}$434.1477, found 434.1477. 


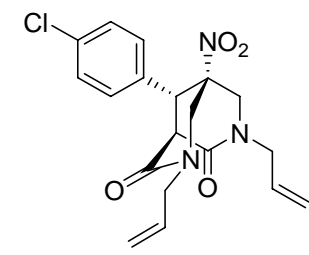

(1R,5R,9R)-3,7-diallyl-9-(4-chlorophenyl)-5-nitro-3,7-diazabicyclo[3.3.1]nonane-2,8-dione: ${ }^{1} \mathrm{H}-\mathrm{NMR}\left(500 \mathrm{MHz}, \mathrm{CDCl}_{3}\right): \delta 7.33$ (m, $2 \mathrm{H}), 7.00$ (m, $2 \mathrm{H}), 5.77$ (m, $2 \mathrm{H}), 5.30$ (m, $4 \mathrm{H}), 4.27$ (m, $1 \mathrm{H})$, 4.15 (m, $2 \mathrm{H}), 4.13$ (m, $1 \mathrm{H}), 4.06$ (m, $1 \mathrm{H}), 3.97$ (dd, $J=7.0,14.5 \mathrm{~Hz}$, $1 \mathrm{H}), 3.81$ (d, $J=2.0 \mathrm{~Hz}, 1 \mathrm{H}), 3.79$ (d, $J=12.8 \mathrm{~Hz}, 1 \mathrm{H}$ ), 3.69 (d, $J=$ $13.1 \mathrm{~Hz}, 1 \mathrm{H}), 3.56$ (dd, $J=1.8,12.8 \mathrm{~Hz}, 1 \mathrm{H}) .13 \mathrm{C}-\mathrm{NMR}(125 \mathrm{MHz}$, $\left.\mathrm{CDCl}_{3}\right): \delta$ 164.5, 163.1, 135.7, 131.9, 131.3, 130.9, 129.9, 129.2, 121.0, 119.9, 85.2, 56.1, 52.7, 50.1, 50.0, 48.8, 45.3. HRMS calc'd for $\mathrm{C}_{19} \mathrm{H}_{21} \mathrm{ClN}_{3} \mathrm{O}_{4}[\mathrm{M}+\mathrm{H}]^{+} 390.1215$, found 390.1213.

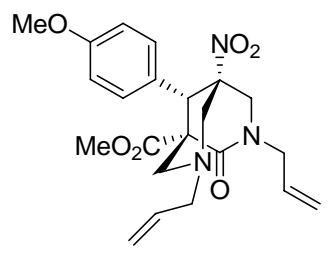

Methyl (1S,5S,9S)-3,7-diallyl-9-(4-methoxyphenyl)-5-nitro-2oxo-3,7-diazabicyclo[3.3.1]nonane-1-carboxylate: 1H-NMR (500 $\left.\mathrm{MHz}, \mathrm{CDCl}_{3}\right): \delta 7.12$ (d, $\left.J=8.9 \mathrm{~Hz}, 2 \mathrm{H}\right), 6.78(\mathrm{~d}, J=8.9 \mathrm{~Hz}, 2 \mathrm{H})$, $5.88(\mathrm{~m}, 1 \mathrm{H}), 5.73(\mathrm{~m}, 1 \mathrm{H}), 5.43(\mathrm{~m}, 1 \mathrm{H}), 5.33(\mathrm{~m}, 1 \mathrm{H}), 5.23$ (m, 1

H), 5.20 (s, $1 \mathrm{H}), 4.57$ (m, $1 \mathrm{H}), 4.11$ (dd, $J=1.9,13.0 \mathrm{~Hz}, 1 \mathrm{H}), 3.83$ (dd, $J=7.0,14.8 \mathrm{~Hz}, 1 \mathrm{H}$ ), 3.77 (s, $4 \mathrm{H}$ ), 3.65 (d, $J=13.0 \mathrm{~Hz}, 1 \mathrm{H}$ ), 3.47 (dd, $J=1.3$, $11.2 \mathrm{~Hz}, 1 \mathrm{H}$ ), 3.34 (s, $3 \mathrm{H}$ ), 3.32 (d, $J=10.3 \mathrm{~Hz}, 1 \mathrm{H}$ ), 3.21 (ddd, $J=1.2$, 6.0, $13.5 \mathrm{~Hz}, 1$ H), 3.06 (dd, $J=0.9,7.0,13.5 \mathrm{~Hz}, 1 \mathrm{H}$ ), 2.98 (dd, $J=1.5,10.3 \mathrm{~Hz}, 1 \mathrm{H}$ ), 2.86 (d, $J=11.2$ $\mathrm{Hz}, 1 \mathrm{H})$. 13C-NMR (125 MHz, $\left.\mathrm{CDCl}_{3}\right): \delta 169.1,166.8,159.9,133.5,131.6,131.2$, 124.8, 119.6, 114.2, 85.3, 65.3, 61.1, 60.1, 56.8, 55.4, 54.0, 52.4, 49.8, 49.5. Anal calc'd for $\mathrm{C}_{22} \mathrm{H}_{27} \mathrm{~N}_{3} \mathrm{O}_{6}$ : C, 61.53; H, 6.34; N, 9.78; Found: C, 61.36; H, 6.32; N, 9.77.

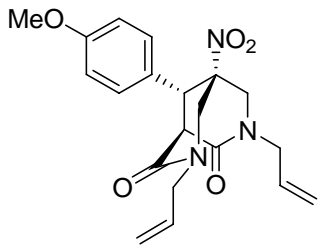

(1R,5R,9R)-3,7-diallyl-5-nitro-9-(4-methoxyphenyl)-3,7diazabicyclo[3.3.1]nonane-2,8-dione: $1 \mathrm{H}-\mathrm{NMR} \quad(500 \mathrm{MHz}$, $\mathrm{CDCl}_{3}$ ): $\delta 6.97(\mathrm{~d}, J=8.8 \mathrm{~Hz}, 2 \mathrm{H}), 6.85(\mathrm{~d}, J=8.8 \mathrm{~Hz}, 2 \mathrm{H}), 5.78$ (m, $2 \mathrm{H}), 5.31$ (m, $3 \mathrm{H}), 5.26$ (m, $1 \mathrm{H}), 4.29$ (dd, $J=6.2,14.6 \mathrm{~Hz}, 1$ H), $4.14(\mathrm{~m}, 3 \mathrm{H}), 4.05$ (dd, $J=6.2,15.1 \mathrm{~Hz}, 1 \mathrm{H}), 3.95$ (dd, $J=7.0$, $14.6 \mathrm{~Hz}, 1 \mathrm{H}), 3.81$ (m, $2 \mathrm{H}$ ), 3.80 (s, $3 \mathrm{H}$ ), 3.68 (d, $J=13.1 \mathrm{~Hz}, 1 \mathrm{H}$ ), 3.52 (dd, $J=1.7$, $12.5 \mathrm{~Hz}, 1 \mathrm{H}) .13 \mathrm{C}-\mathrm{NMR}\left(125 \mathrm{MHz}, \mathrm{CDCl}_{3}\right): \delta 164.9,163.5,160.3,131.4,131.2,129.0$, 125.1, 120.7, 119.7, 115.0, 85.7, 56.0, 55.0, 53.0, 50.09, 50.05, 48.8, 45.3. HRMS calc'd for $\mathrm{C}_{20} \mathrm{H}_{24} \mathrm{~N}_{3} \mathrm{O}_{5}[\mathrm{M}+\mathrm{H}]^{+}$386.1711, found 386.1714. Anal calc'd for $\mathrm{C}_{20} \mathrm{H}_{23} \mathrm{~N}_{3} \mathrm{O}_{5}$ : C, 62.33; H, 6.01; N, 10.90; Found: C, 62.26; H, 6.58; N, 10.98 .

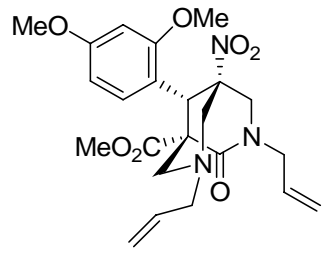

Methyl (1S,5S,9S)-3,7-diallyl-9-(2,4-dimethoxyphenyl)-5-nitro-2oxo-3,7-diazabicyclo[3.3.1]nonane-1-carboxylate: 1H-NMR (500 $\mathrm{MHz}, \mathrm{CDCl}_{3}$ ): $\delta 7.13$ (d, $\left.J=9.0 \mathrm{~Hz}, 1 \mathrm{H}\right), 6.41$ (m, $\left.2 \mathrm{H}\right), 5.87$ (m, 1 H), 5.76 (m, $1 \mathrm{H}), 5.42$ (dd, $J=1.3,17.1 \mathrm{~Hz}, 1 \mathrm{H}), 5.33$ (d, $J=17.1$ Hz, $1 \mathrm{H}$ ), 5.23 (dd, $J=1.1,9.1 \mathrm{~Hz}, 1 \mathrm{H}), 5.21$ (s, $1 \mathrm{H}), 4.56$ (s, $1 \mathrm{H}$ ), 4.48 (dd, $J=4.9,14.7 \mathrm{~Hz}, 1 \mathrm{H}), 4.32$ (d, $J=13.0 \mathrm{~Hz}, 1 \mathrm{H}), 3.80$ (dd, $J=5.8,14.0 \mathrm{~Hz}, 1 \mathrm{H}$ ), 3.77 (s, $3 \mathrm{H}$ ), 3.76 (s, $3 \mathrm{H}$ ), 3.66 (d, $J=13.0 \mathrm{~Hz}, 1 \mathrm{H}), 3.45$ (dd, $J=$ 1.2, $11.3 \mathrm{~Hz}, 1 \mathrm{H}$ ), 3.35 (s, $3 \mathrm{H}$ ), 3.30 (dd, $J=0.9,10.2 \mathrm{~Hz}, 1 \mathrm{H}$ ), 3.23 (dd, $J=6.0,13.5$ Hz, $1 \mathrm{H}$ ), 3.09 (dd, $J=6.9,13.5 \mathrm{~Hz}, 1 \mathrm{H}$ ), 3.03 (dd, $J=1.3,10.2 \mathrm{~Hz}, 1 \mathrm{H}$ ), 2.92 (d, $J=$ 
$11.3 \mathrm{~Hz}, 1 \mathrm{H})$. 13C-NMR (125 MHz, $\left.\mathrm{CDCl}_{3}\right): \delta$ 169.1, 167.2, 160.7, 159.4, 133.47, 131.6, 129.1, 119.7, 119.6, 114.8, 104.8, 98.9, 84.8, 65.7, 60.9, 60.3, 56.7, 56.2, 55.4, 52.2, 50.5, 49.8, 43.8. HRMS calc'd for $\mathrm{C}_{23} \mathrm{H}_{30} \mathrm{~N}_{3} \mathrm{O}_{7}[\mathrm{M}+\mathrm{H}]^{+} 460.2078$, found 460.2068.

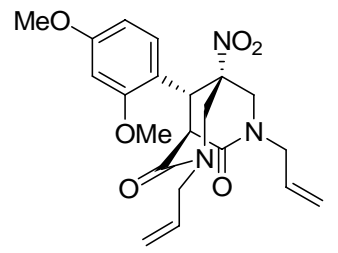

(1R,5R,9R)-3,7-diallyl-5-nitro-9-(2,4-dimethoxyphenyl)-3,7diazabicyclo[3.3.1]nonane-2,8-dione: $1_{\mathrm{H}-\mathrm{NMR}} \quad(500 \mathrm{MHz}$, $\left.\mathrm{CDCl}_{3}\right): \delta 6.82(\mathrm{~d}, J=9.2 \mathrm{~Hz}, 1 \mathrm{H}), 6.42(\mathrm{~m}, 2 \mathrm{H}), 5.78(\mathrm{~m}, 2 \mathrm{H})$, 5.29 (m, $4 \mathrm{H}$ ), 4.04 (s, $1 \mathrm{H}), 4.37$ (d, $J=13.0 \mathrm{~Hz}, 1 \mathrm{H}), 4.25$ (dd, $J=$ 6.3, $14.6 \mathrm{~Hz}, 1 \mathrm{H}), 4.16(\mathrm{~m}, 1 \mathrm{H}), 4.14$ (d, $J=12.1 \mathrm{~Hz}, 1 \mathrm{H}), 4.07$ (d, $J=6.3,15.0 \mathrm{~Hz}, 1 \mathrm{H}$ ), 3.92 (dd, $J=7.0,14.5 \mathrm{~Hz}, 1 \mathrm{H}$ ), 3.80 (s, $3 \mathrm{H}$ ), 3.72 (d, $J=2.0 \mathrm{~Hz}$, $1 \mathrm{H}$ ), 3.71 (s, $3 \mathrm{H}$ ), 3.60 (d, $J=12.9 \mathrm{~Hz}, 1 \mathrm{H}$ ), 3.45 (dd, $J=1.5,12.7 \mathrm{~Hz}, 1 \mathrm{H}$ ). 13C-NMR (125 MHz, $\left.\mathrm{CDCl}_{3}\right): \delta 165.4,163.8,161.4,159.1,131.7,131.5,128.7,120.6,119.6,114.3$, 105.1, 99.1, 85.5, 56.2, 55.6, 55.4, 53.3, 50.9, 49.8, 49.0, 40.9. HRMS calc'd for $\mathrm{C}_{21} \mathrm{H}_{26} \mathrm{~N}_{3} \mathrm{O}_{6}[\mathrm{M}+\mathrm{H}]^{+}$416.1816, found 416.1831.

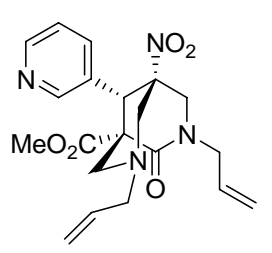

Methyl (1S,5S,9S)-3,7-diallyl-5-nitro-2-oxo-9-pyridin-3-yl-3,7diazabicyclo[3.3.1]nonane-1-carboxylate: $1 \mathrm{H}-\mathrm{NMR} \quad(500 \mathrm{MHz}$, $\mathrm{CDCl}_{3}$ ): $\delta 8.54$ (d, $J=4.3 \mathrm{~Hz}, 1 \mathrm{H}$ ), 8.47 (d, $J=1.9 \mathrm{~Hz}, 1 \mathrm{H}$ ), 7.58 (d, $J$ $=8.0 \mathrm{~Hz}, 1 \mathrm{H}), 7.23(\mathrm{dd}, J=4.8,8.0 \mathrm{~Hz}, 1 \mathrm{H}), 5.91(\mathrm{~m}, 1 \mathrm{H}), 5.73(\mathrm{~m}, 1$ H), $5.44(\mathrm{~m}, 1 \mathrm{H}), 5.36(\mathrm{~m}, 1 \mathrm{H}), 5.24(\mathrm{~m}, 2 \mathrm{H}), 4.55$ (dd, $J=5.5,14.8$ $\mathrm{Hz}, 1 \mathrm{H}$ ), 4.08 (dd, $J=1.7,13.2 \mathrm{~Hz}, 1 \mathrm{H}$ ), 3.85 (dd, $J=6.9,14.8 \mathrm{~Hz}, 1$ H), 3.82 (s, $1 \mathrm{H}$ ), 3.74 (d, $J=13.2 \mathrm{~Hz}, 1 \mathrm{H}), 3.53$ (d, $J=11.3 \mathrm{~Hz}, 1 \mathrm{H}), 3.37$ (d, $J=10.4$ Hz, 1 H), 3.34 (s, 3 H), 3.23 (dd, $J=6.0,13.5 \mathrm{~Hz}, 1 \mathrm{H}$ ), 3.09 (dd, $J=6.9,13.5 \mathrm{~Hz}, 1 \mathrm{H}$ ), 2.97 (dd, $J=1.8,10.4 \mathrm{~Hz}, 1 \mathrm{H}$ ), 2.87 (d, $J=11.4 \mathrm{~Hz}, 1 \mathrm{H}$ ). 13C-NMR (125 MHz, $\left.\mathrm{CDCl}_{3}\right): \delta 168.6,166.4,151.3,150.2,137.2,133.2,131.2,129.3,123.5,120.0,119.9$, 84.9, 65.3, 60.8, 60.1, 56.6, 52.5, 52.1, 49.9, 49.5. HRMS calc'd for $\mathrm{C}_{20} \mathrm{H}_{25} \mathrm{~N}_{4} \mathrm{O}_{5}[\mathrm{M}+\mathrm{H}]^{+}$ 401.1820, found 401.1815.

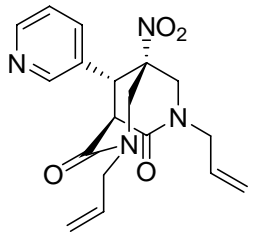

(1R,5R,9R)-3,7-diallyl-5-nitro-9-pyridin-3-yl-3,7-diazabicyclo[3.3.1]nonane-2,8-dione: $1_{\mathrm{H}-\mathrm{NMR}}\left(500 \mathrm{MHz}, \mathrm{CDCl}_{3}\right)$ : $\delta 8.62$ (dd, $J=1.5,4.8$ $\mathrm{Hz}, 1 \mathrm{H}$ ), 8.38 (d, $J=2.4 \mathrm{~Hz}, 1 \mathrm{H}), 7.40$ (m, $1 \mathrm{H}), 7.31$ (dd, $J=4.8,7.9$ Hz, $1 \mathrm{H}$ ), 5.77 (m, $2 \mathrm{H}), 5.32$ (m, $4 \mathrm{H}), 4.29$ (m, $1 \mathrm{H}), 4.2$ (s, $1 \mathrm{H}), 4.16$ (dd, $J=1.4,13.1 \mathrm{~Hz}, 1 \mathrm{H}$ ), 4.08 (m, $2 \mathrm{H}), 3.97$ (dd, $J=7.0,14.6 \mathrm{~Hz}, 1$ H), 3.85 (dd, $J=1.0,12.9 \mathrm{~Hz}, 1 \mathrm{H}), 3.81$ (d, $J=2.1 \mathrm{~Hz}, 1 \mathrm{H}), 3.73$ (d, $J=13.1 \mathrm{~Hz}, 1 \mathrm{H}$ ), 3.65 (dd, $J=1.7,13.1 \mathrm{~Hz}, 1 \mathrm{H}) .13 \mathrm{C}-\mathrm{NMR}\left(125 \mathrm{MHz}, \mathrm{CDCl}_{3}\right)$ : $\delta 164.2,162.7,150.9$, 149.4, 135.4, 131.2, 130.8, 129.5, 124.3, 121.1, 120.0, 84.9, 56.3, 52.5, 50.1, 50.0, 48.9, 43.9. HRMS calc'd for $\mathrm{C}_{18} \mathrm{H}_{21} \mathrm{~N}_{4} \mathrm{O}_{4}[\mathrm{M}+\mathrm{H}]^{+}$357.1557, found 357.1551.

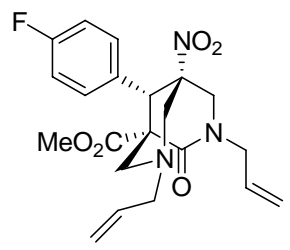

Methyl (1S,5S,9S)-3,7-diallyl-9-(4-fluorophenyl)-5-nitro-2-oxo-3,7diazabicyclo[3.3.1]nonane-1-carboxylate: $1 \mathrm{H}-\mathrm{NMR} \quad(500 \mathrm{MHz}$, $\mathrm{CDCl}_{3}$ ): $\delta 7.20$ (dd, $\left.J=8.4,11.9 \mathrm{~Hz}, 2 \mathrm{H}\right), 6.96(\mathrm{t}, J=8.5 \mathrm{~Hz}, 2 \mathrm{H})$, 
5.88 (m, $1 \mathrm{H}), 5.73$ (m $1 \mathrm{H}), 5.43$ (dd, $J=1.3,17.3 \mathrm{~Hz}, 1 \mathrm{H}$ ), 5.34 (dd, $J=0.9,10.2 \mathrm{~Hz}, 1$ H), 5.23 (m, 1 H), 5.21 (s, 1 H), 4.54 (dd, $J=5.5,14.8 \mathrm{~Hz}, 1 \mathrm{H}$ ), 4.09 (dd, $J=2.0,13.1$ Hz, $1 \mathrm{H}$ ), 3.85 (dd, $J=7.0,14.8 \mathrm{~Hz}, 1 \mathrm{H}), 3.81$ (s, $1 \mathrm{H}), 3.67$ (d, $J=13.1 \mathrm{~Hz}, 1 \mathrm{H}), 3.48$ (dd, $J=1.3,11.3 \mathrm{~Hz}, 1 \mathrm{H}$ ), 3.34 (s, $3 \mathrm{H}$ ), 3.33 (dd, $J=1.1,10.2 \mathrm{~Hz}, 1 \mathrm{H}$ ), 3.21 (dd, $J=$ 6.1, $13.5 \mathrm{~Hz}, 1 \mathrm{H}$ ), 3.08 (dd, $J=7.0,13.5 \mathrm{~Hz}, 1 \mathrm{H}$ ), 2.98 (dd, $J=1.8,10.4 \mathrm{~Hz}, 1 \mathrm{H}$ ), 2.86 (d, $J=11.3 \mathrm{~Hz}, 1 \mathrm{H}$ ). 13C-NMR (125 MHz, $\mathrm{CDCl}_{3}$ ): $\delta$ 168.9, 166.5, 162.9 (d, $J=247.3$ $\mathrm{Hz}), 133.2,131.8$ (d, $J=7.4 \mathrm{~Hz}), 131.3,128.8$ (d, $J=3.4 \mathrm{~Hz}), 119.8,115.8$ (d, $J=21.3$ $\mathrm{Hz})$, 85.1, 65.2, 60.9, 60.1, 56.7, 53.8, 52.4, 49.8, 49.4. HRMS calc'd for $\mathrm{C}_{21} \mathrm{H}_{25} \mathrm{FN}_{3} \mathrm{O}_{5}$ $[\mathrm{M}+\mathrm{H}]^{+}$418.1773, found 418.1780 .

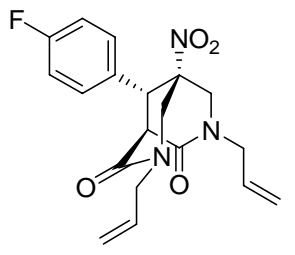

(1R,5R,9R)-3,7-diallyl-9-(4-fluorophenyl)-5-nitro-3,7-diazabicyclo[3.3.1]nonane-2,8-dione: $1_{\mathrm{H}-\mathrm{NMR}}\left(500 \mathrm{MHz}, \mathrm{CDCl}_{3}\right): \delta 7.05$ (d, $J=$ $6.6 \mathrm{~Hz}, 4 \mathrm{H}), 5.78(\mathrm{~m}, 2 \mathrm{H}), 5.31(\mathrm{~m}, 4 \mathrm{H}), 4.28(\mathrm{~m}, 1 \mathrm{H}), 4.16(\mathrm{~m}, 2 \mathrm{H})$, $4.12(\mathrm{~m}, 1 \mathrm{H}), 4.07$ (m, $1 \mathrm{H}), 3.96$ (dd, $J=7.0,14.5 \mathrm{~Hz}, 1 \mathrm{H}), 3.82$ (d, $J$ $=2.0 \mathrm{~Hz}, 1 \mathrm{H}), 3.80(\mathrm{~m}, 1 \mathrm{H}), 3.69(\mathrm{~d}, J=13.1 \mathrm{~Hz}, 1 \mathrm{H}), 3.56(\mathrm{dd}, J=$ 1.7, $12.7 \mathrm{~Hz}, 1 \mathrm{H})$.13C-NMR (125 MHz, $\left.\mathrm{CDCl}_{3}\right): \delta 164.5,163.2$ (d, $J$ = 249.9 Hz), 163.1, 131.3, 130.9, $129.6(\mathrm{~d}, J=8.3 \mathrm{~Hz}), 129.2(\mathrm{~d}, J=3.3 \mathrm{~Hz}), 120.9$, 119.8, 116.7 (d, $J=21.7 \mathrm{~Hz}), 85.4,56.1,52.8,50.1,49.9$, 48.8, 45.2. HRMS calc'd for $\mathrm{C}_{19} \mathrm{H}_{21} \mathrm{FN}_{3} \mathrm{O}_{4}[\mathrm{M}+\mathrm{H}]^{+}$374.1511, found 374.1509. 

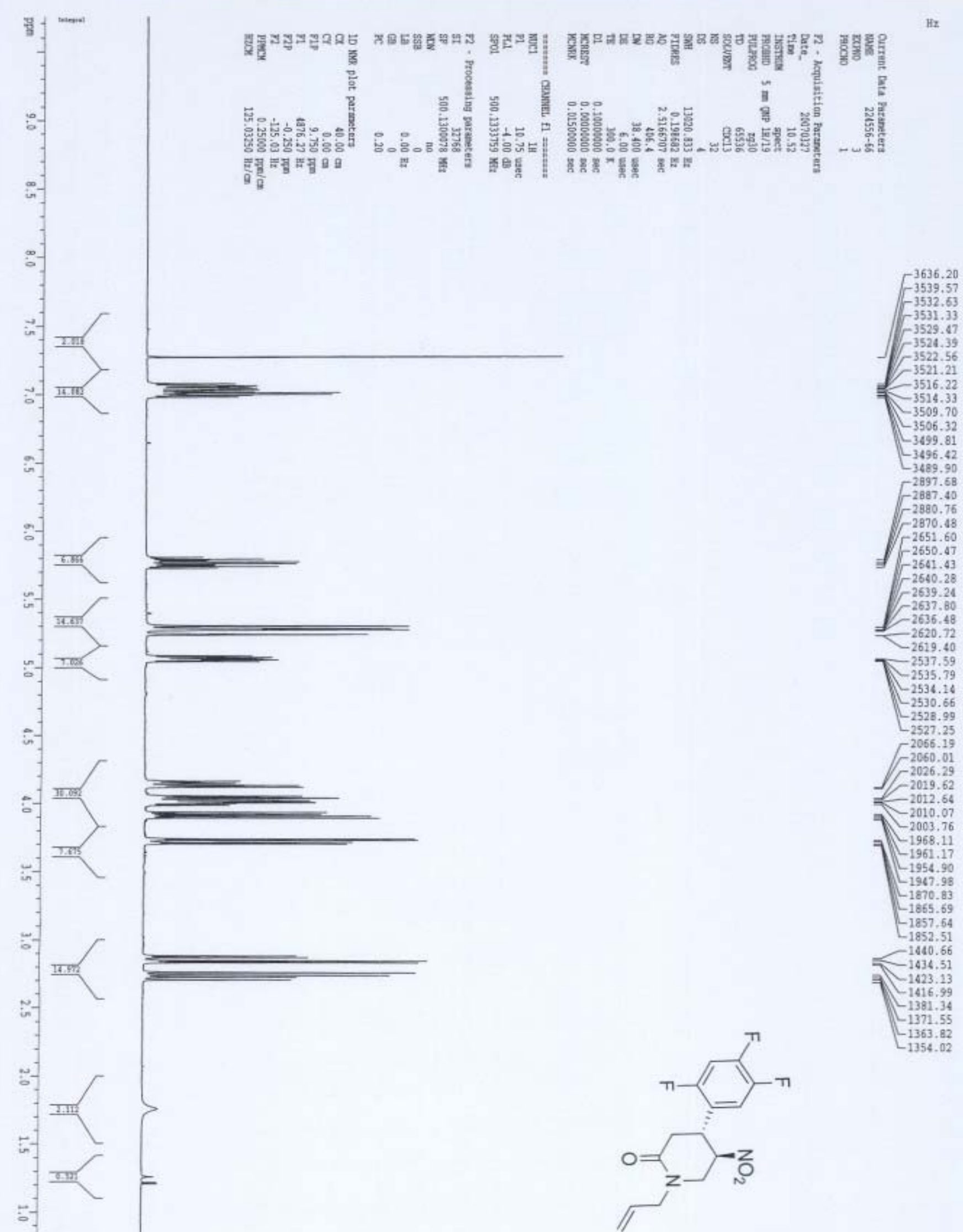


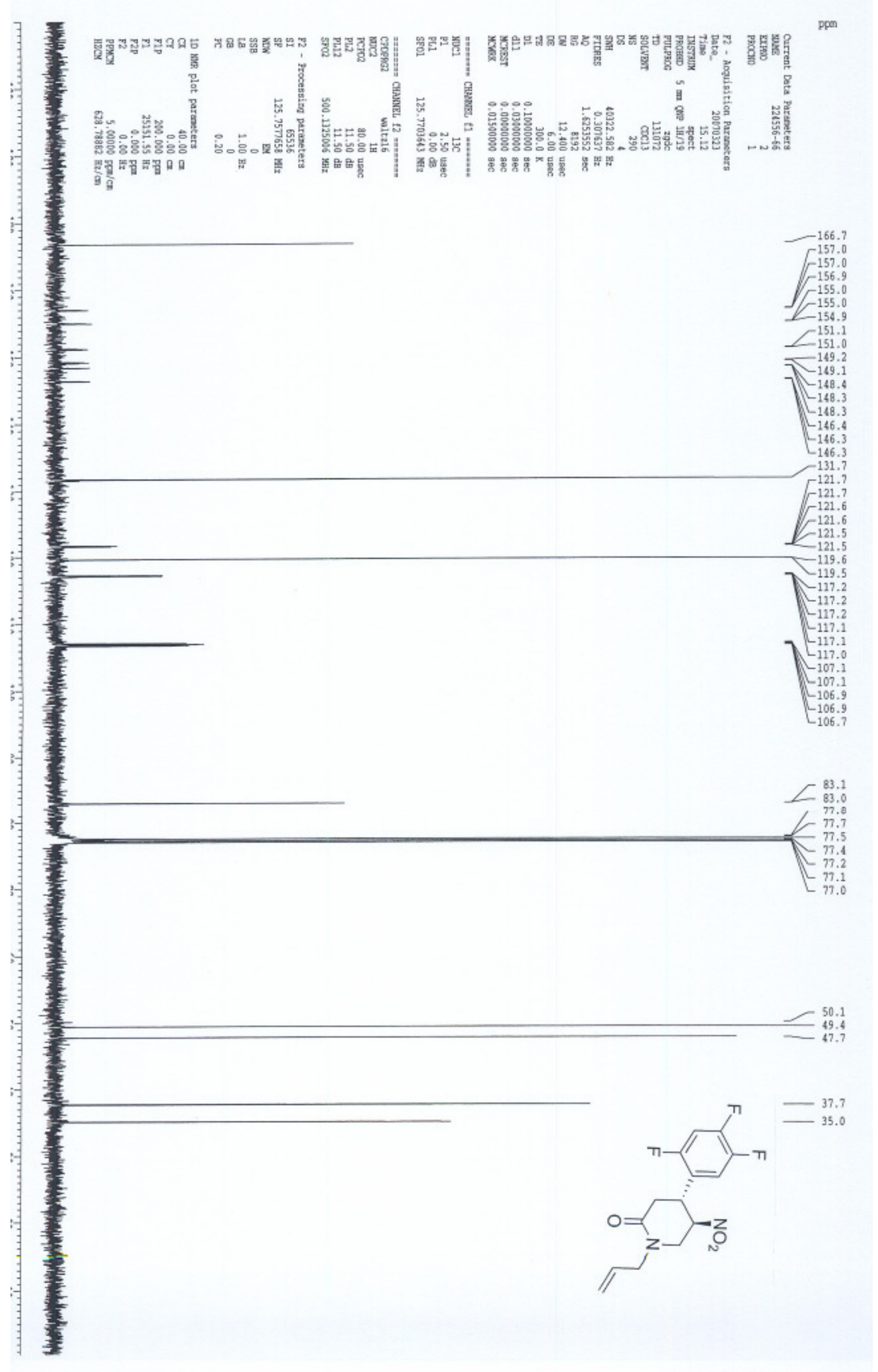




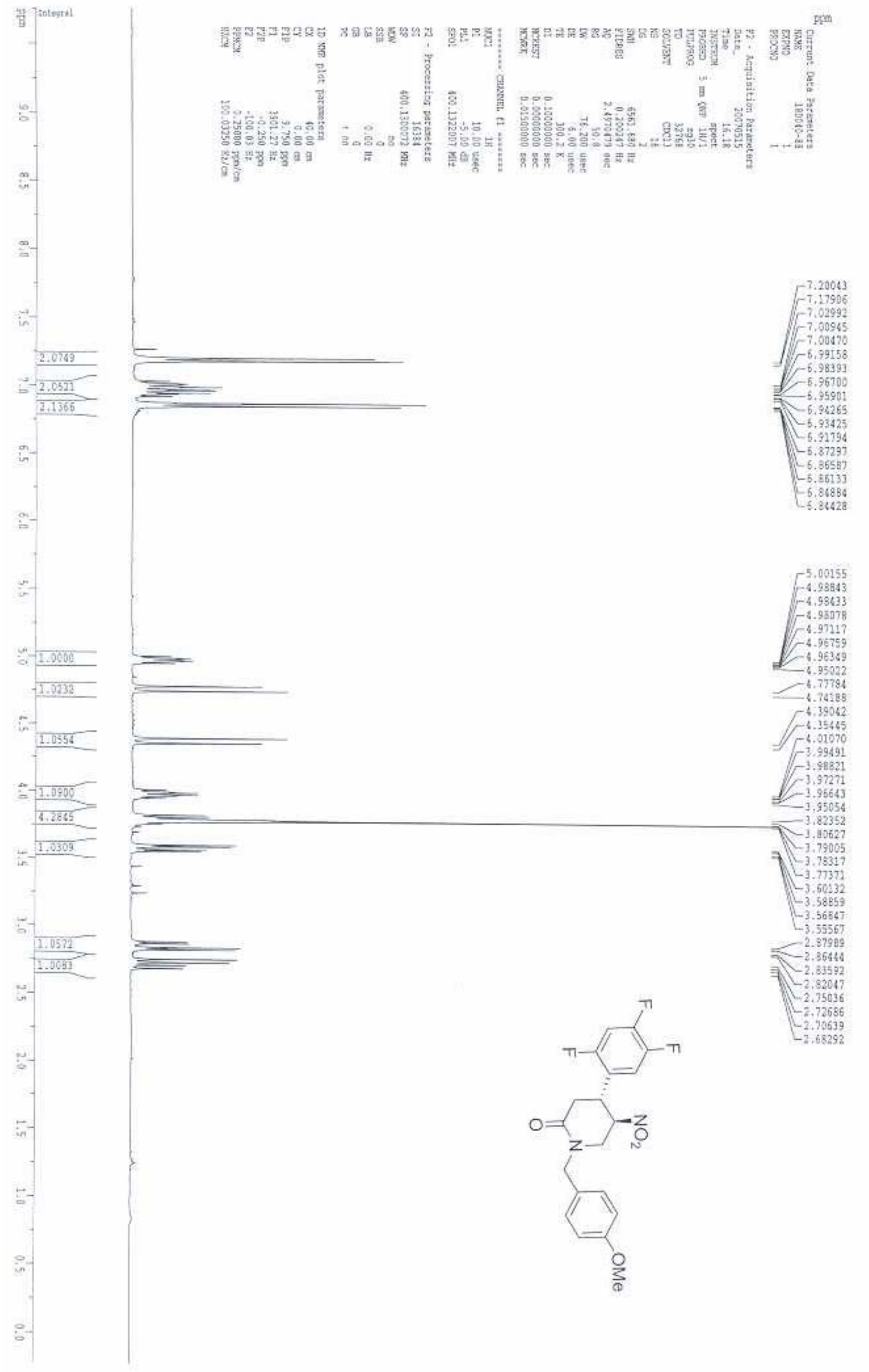




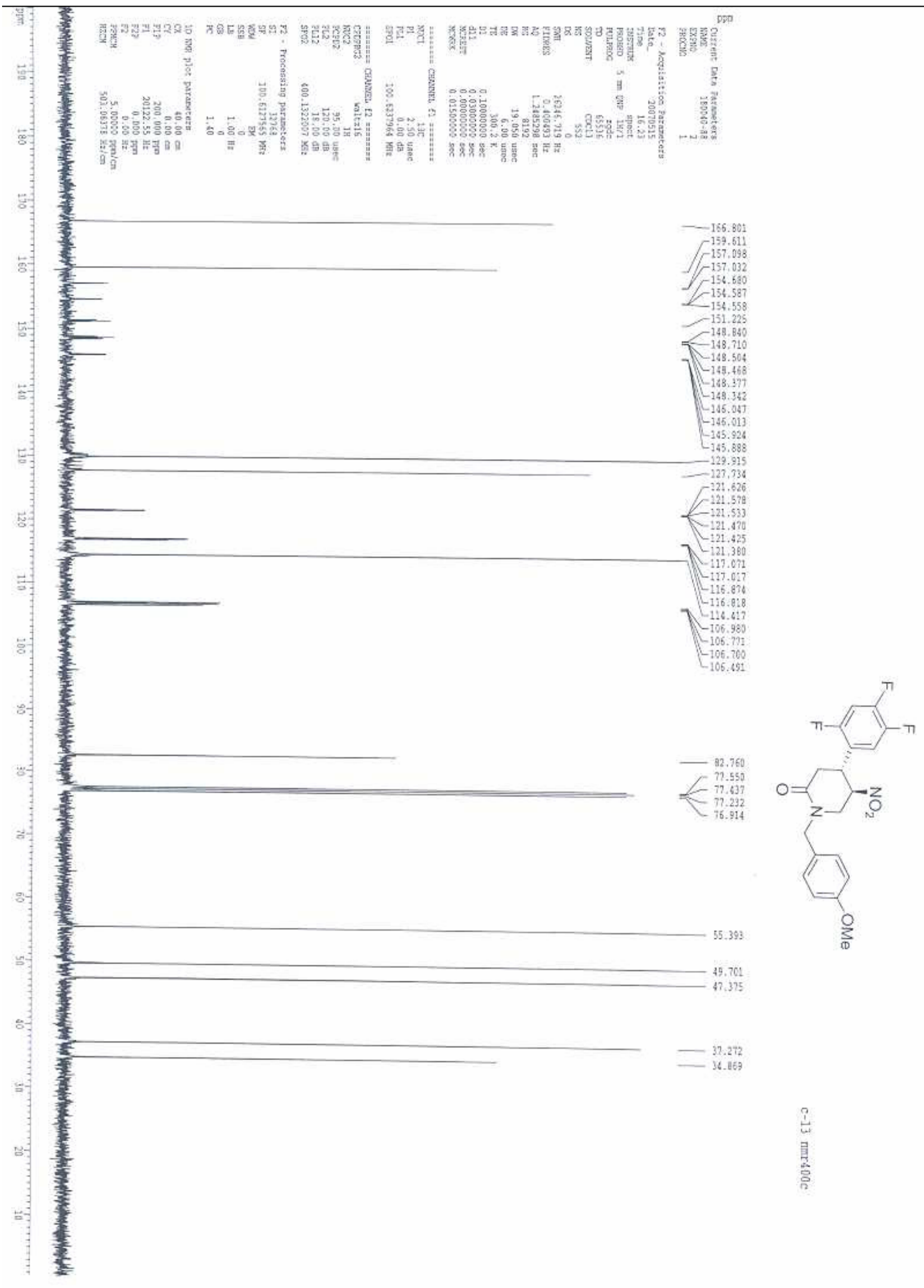




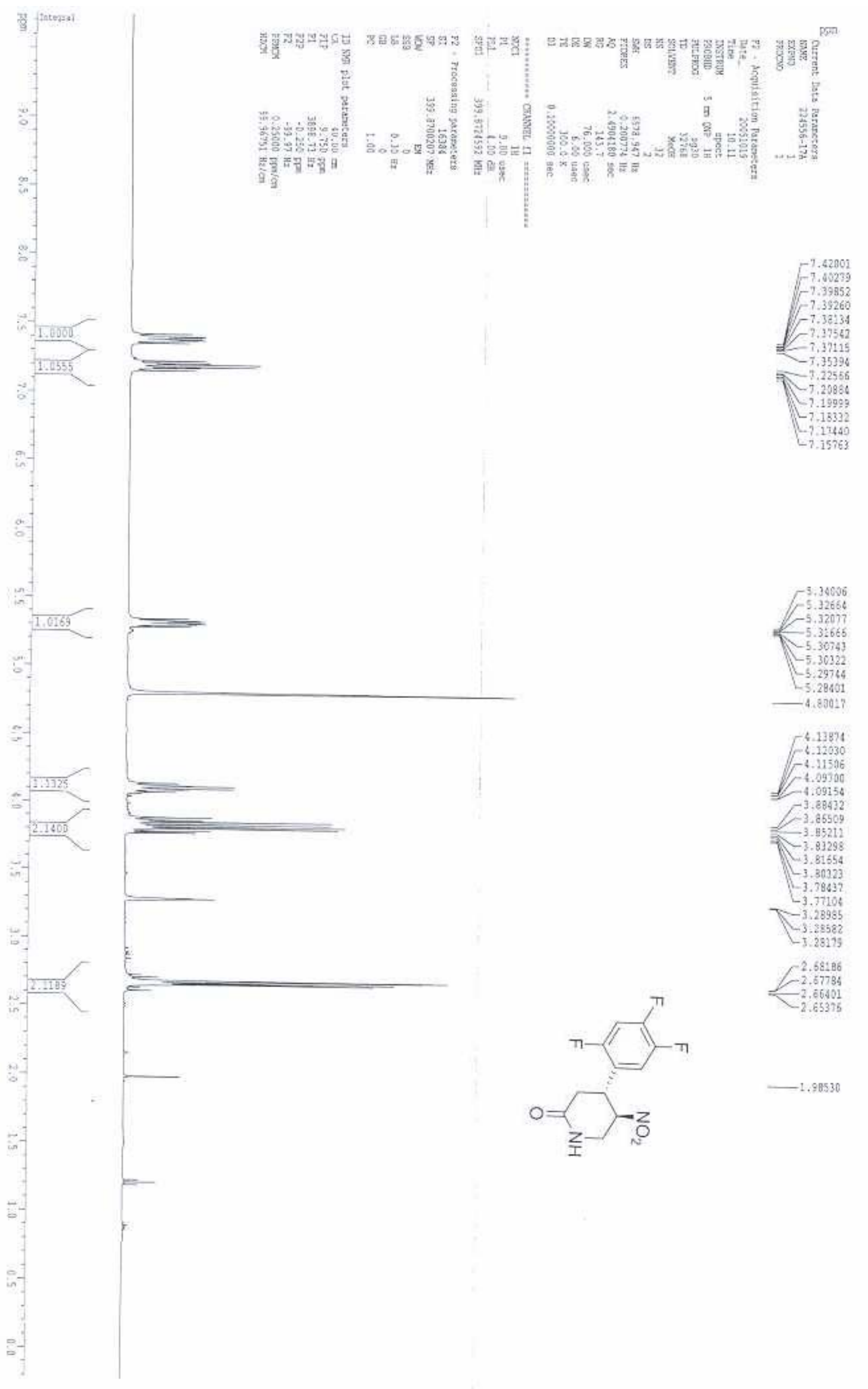




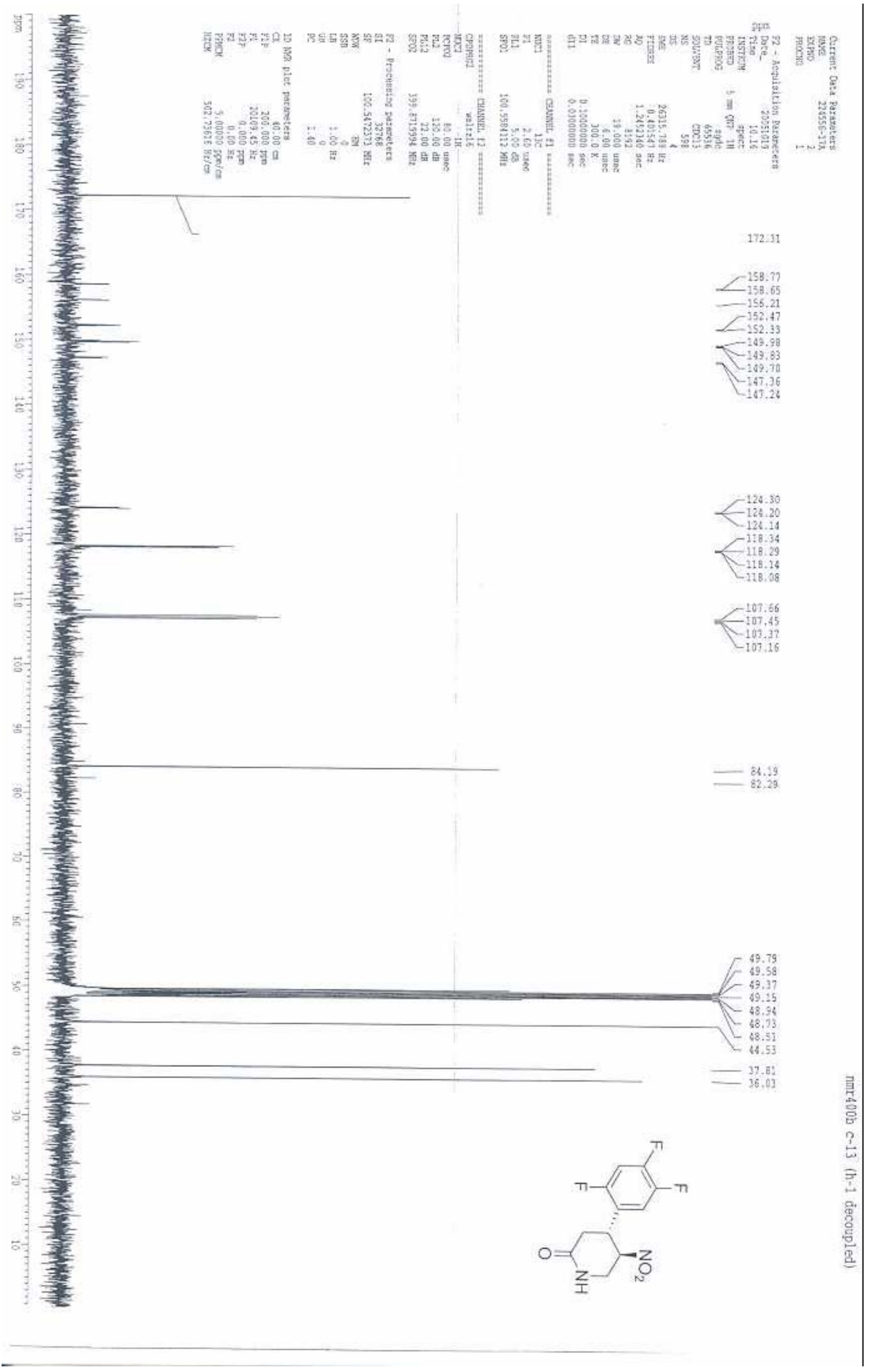




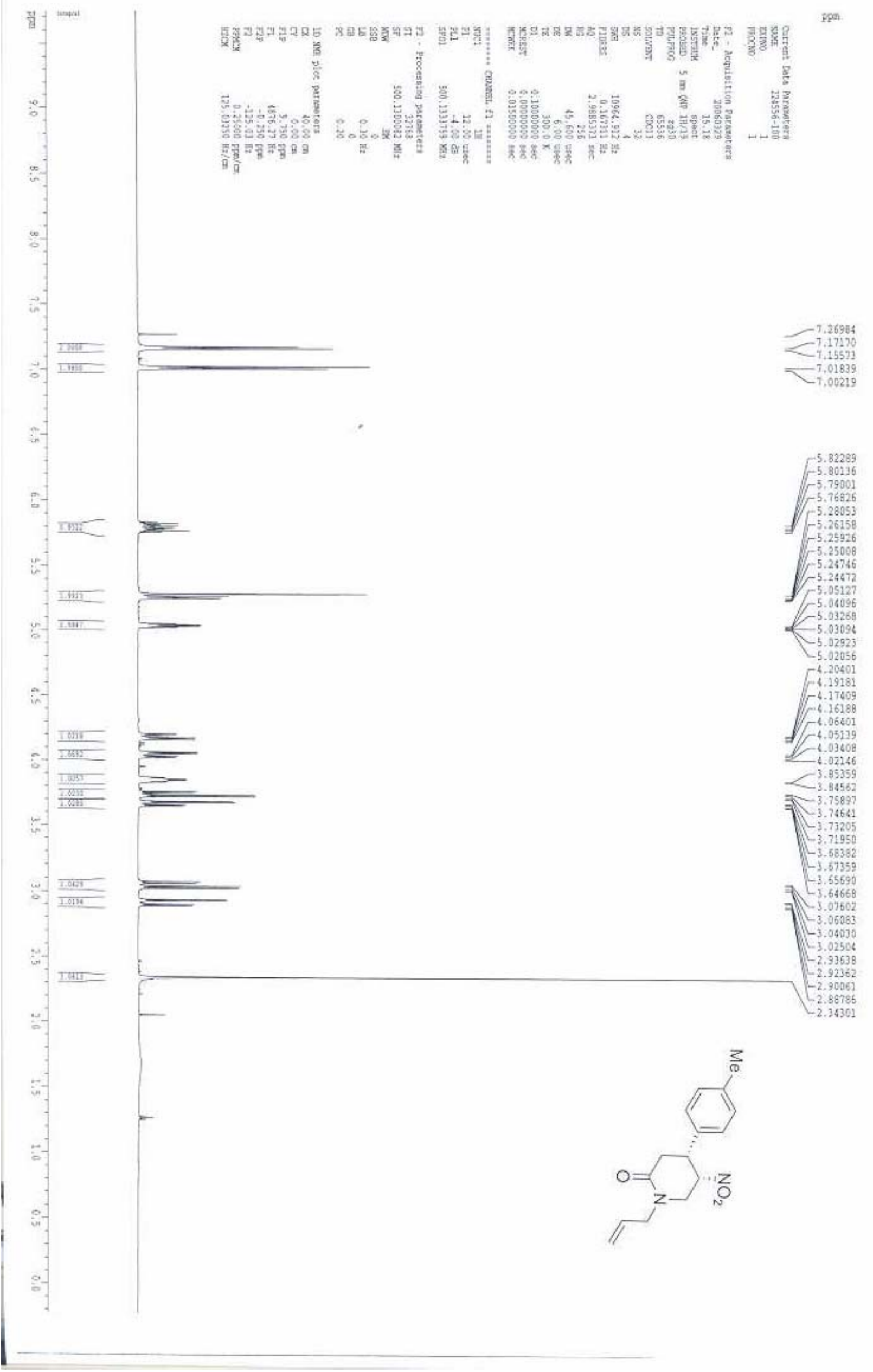




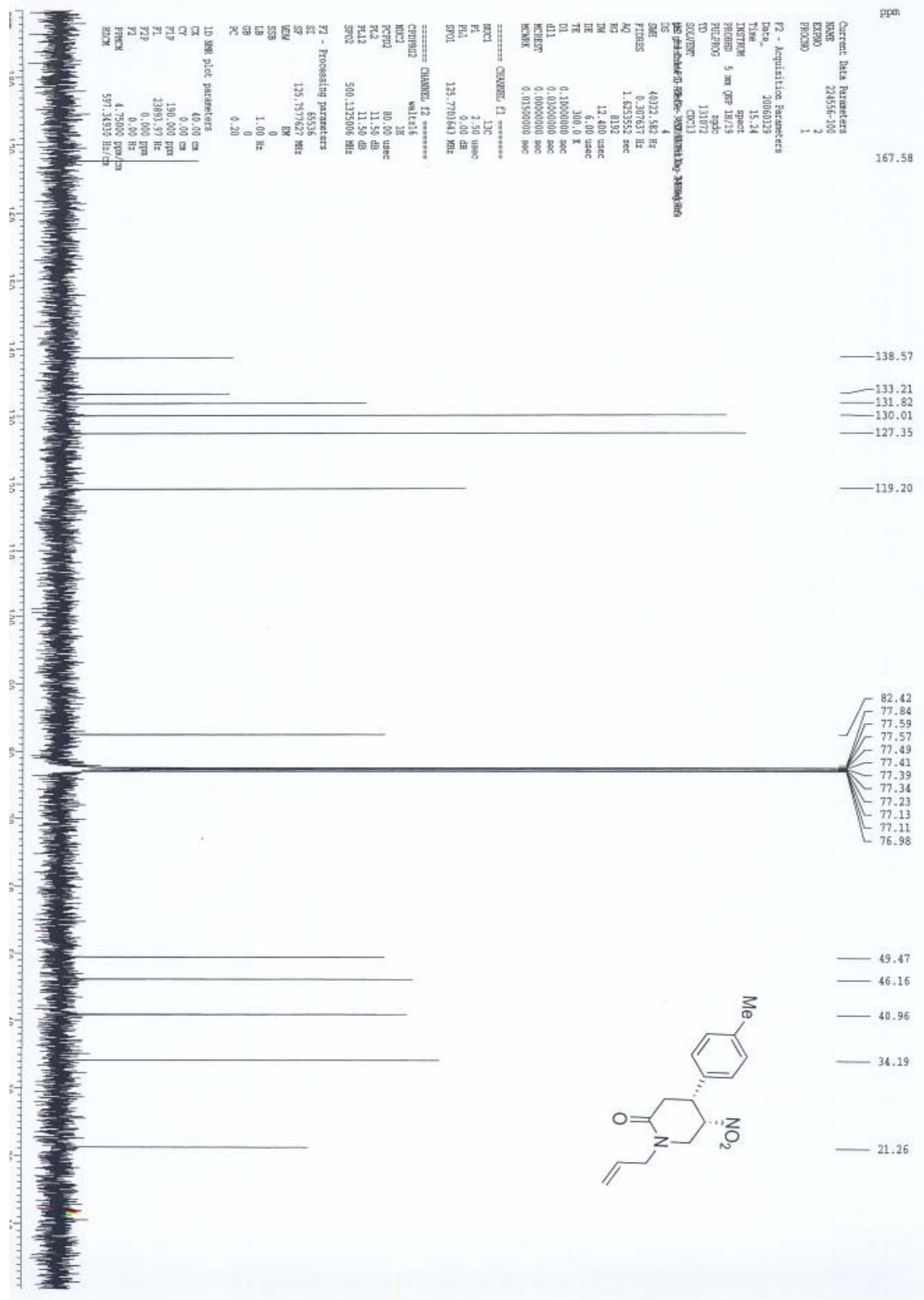




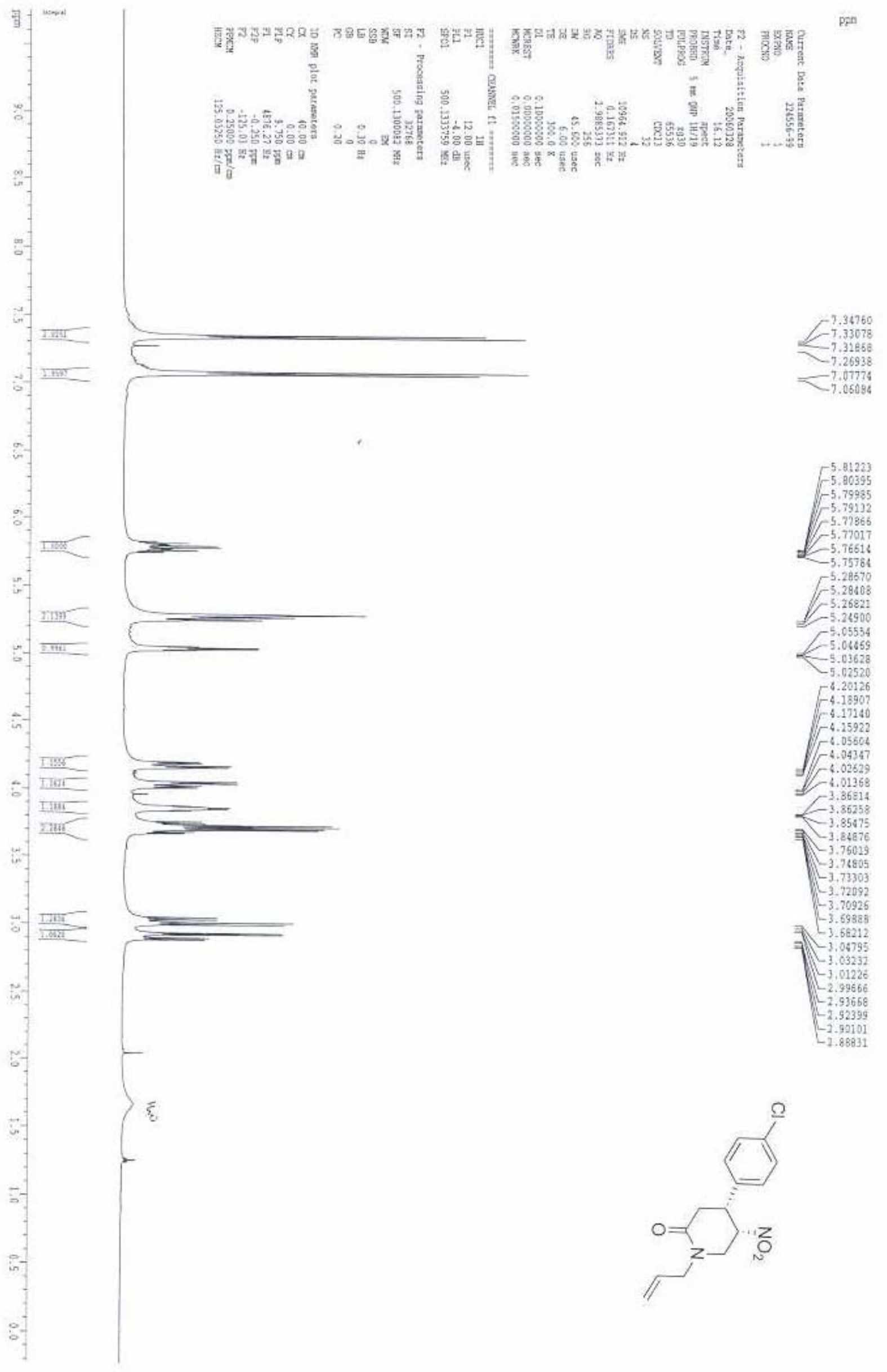



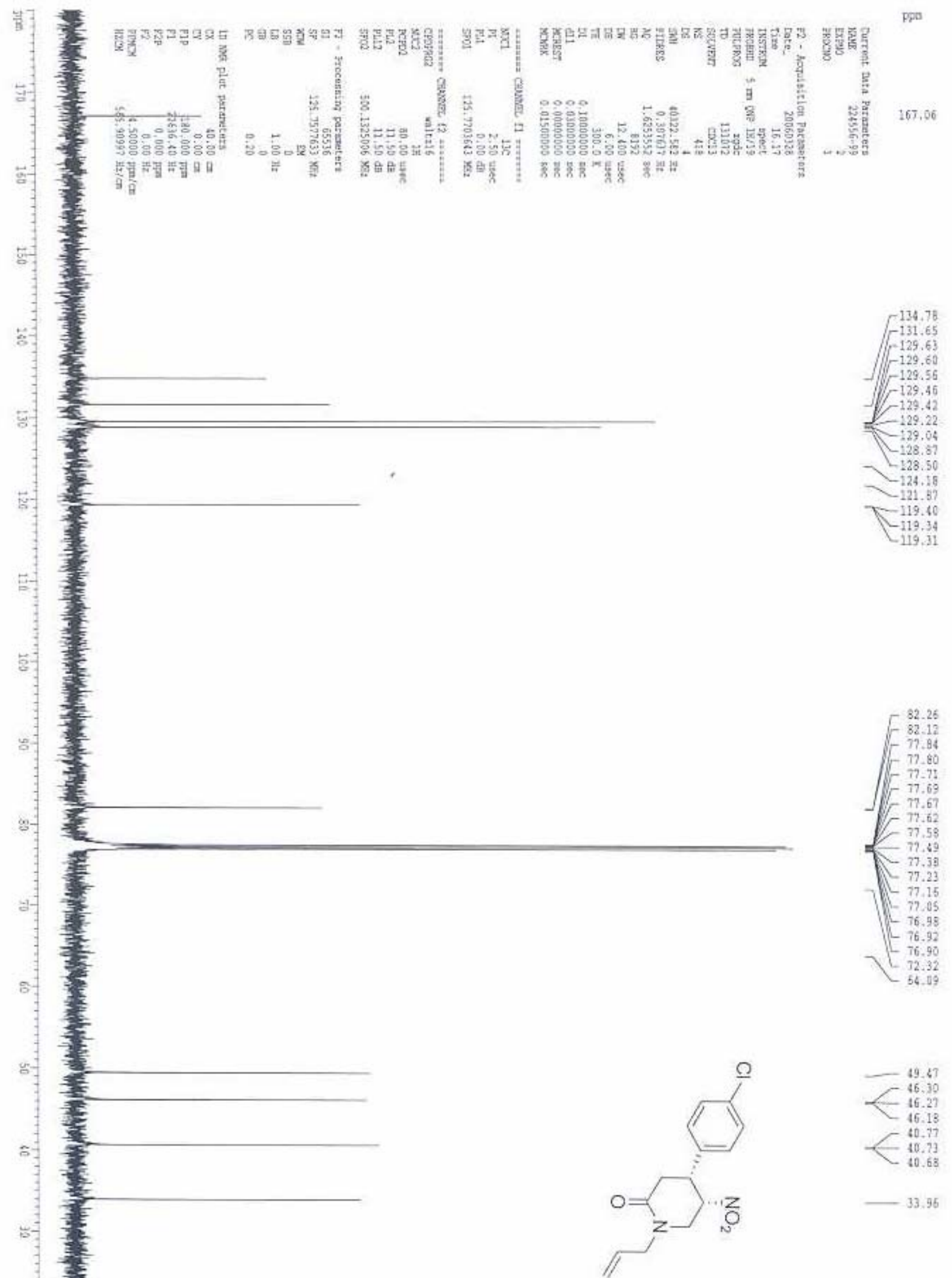

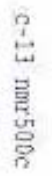




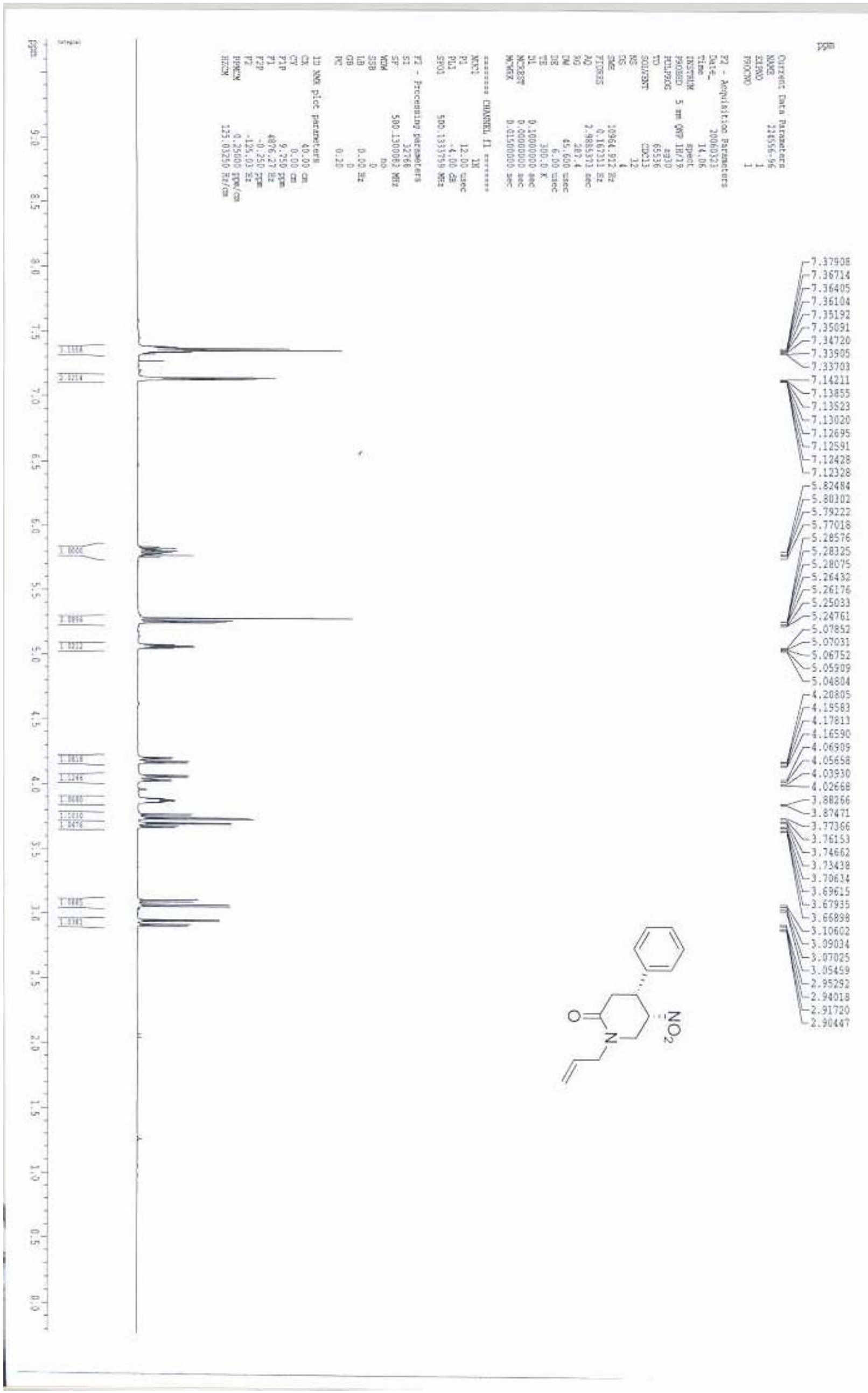




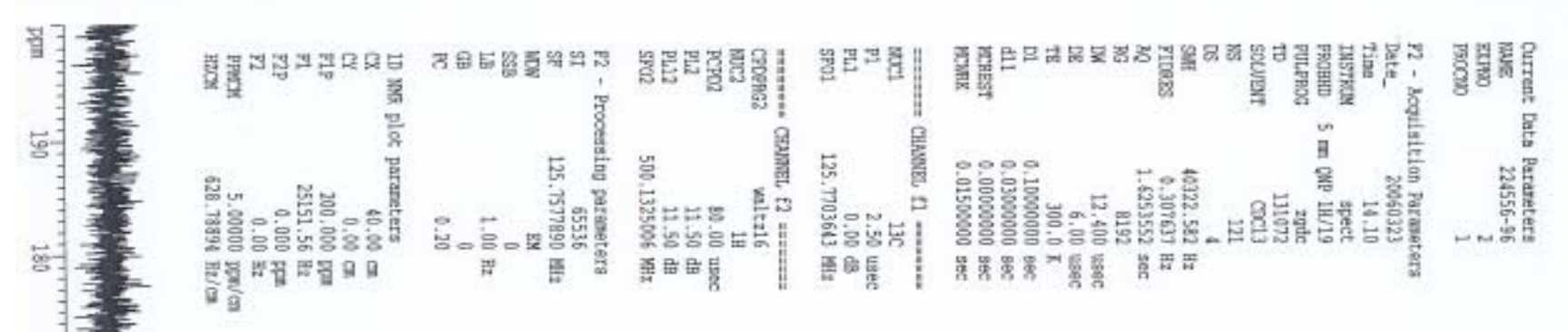




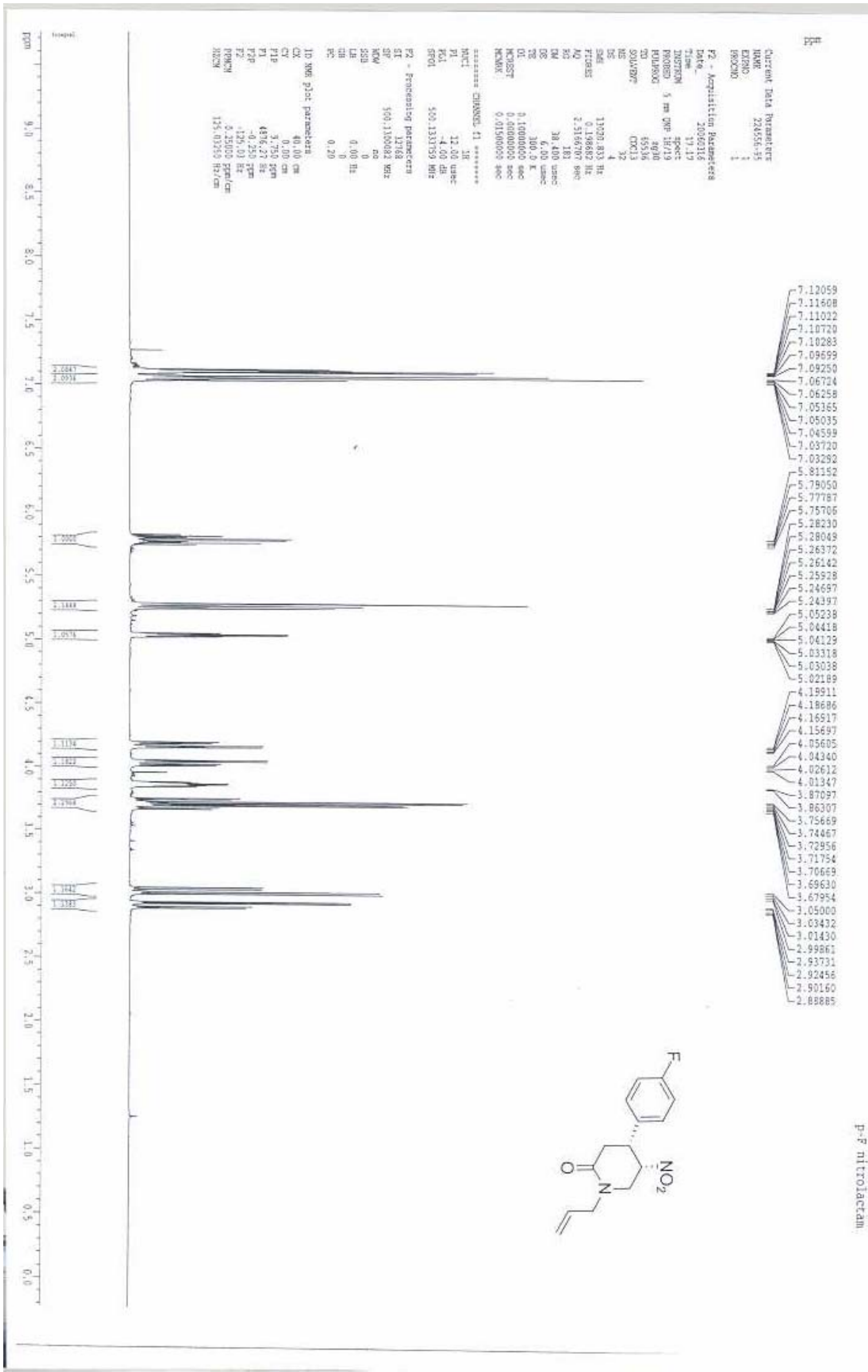

몸. 


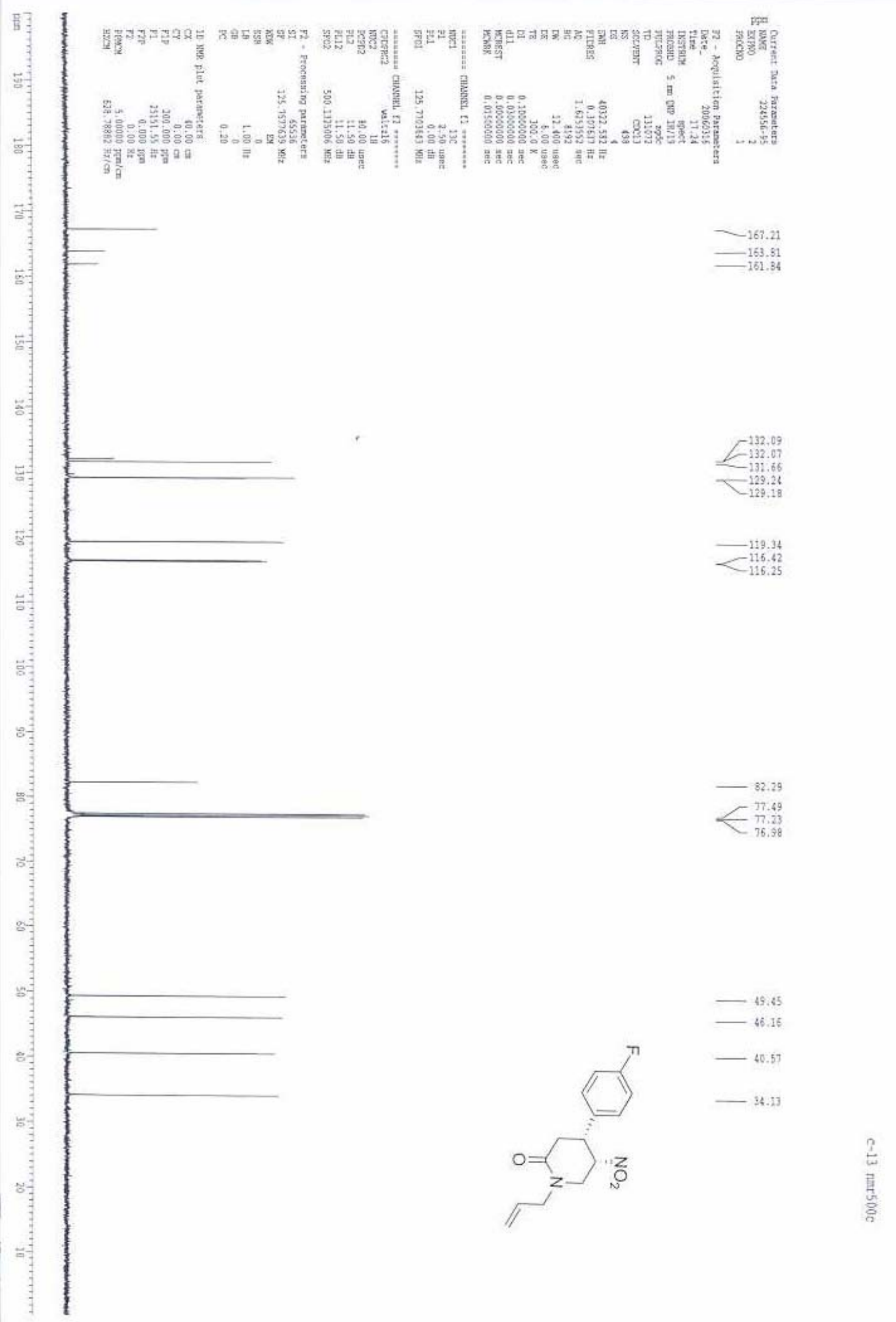




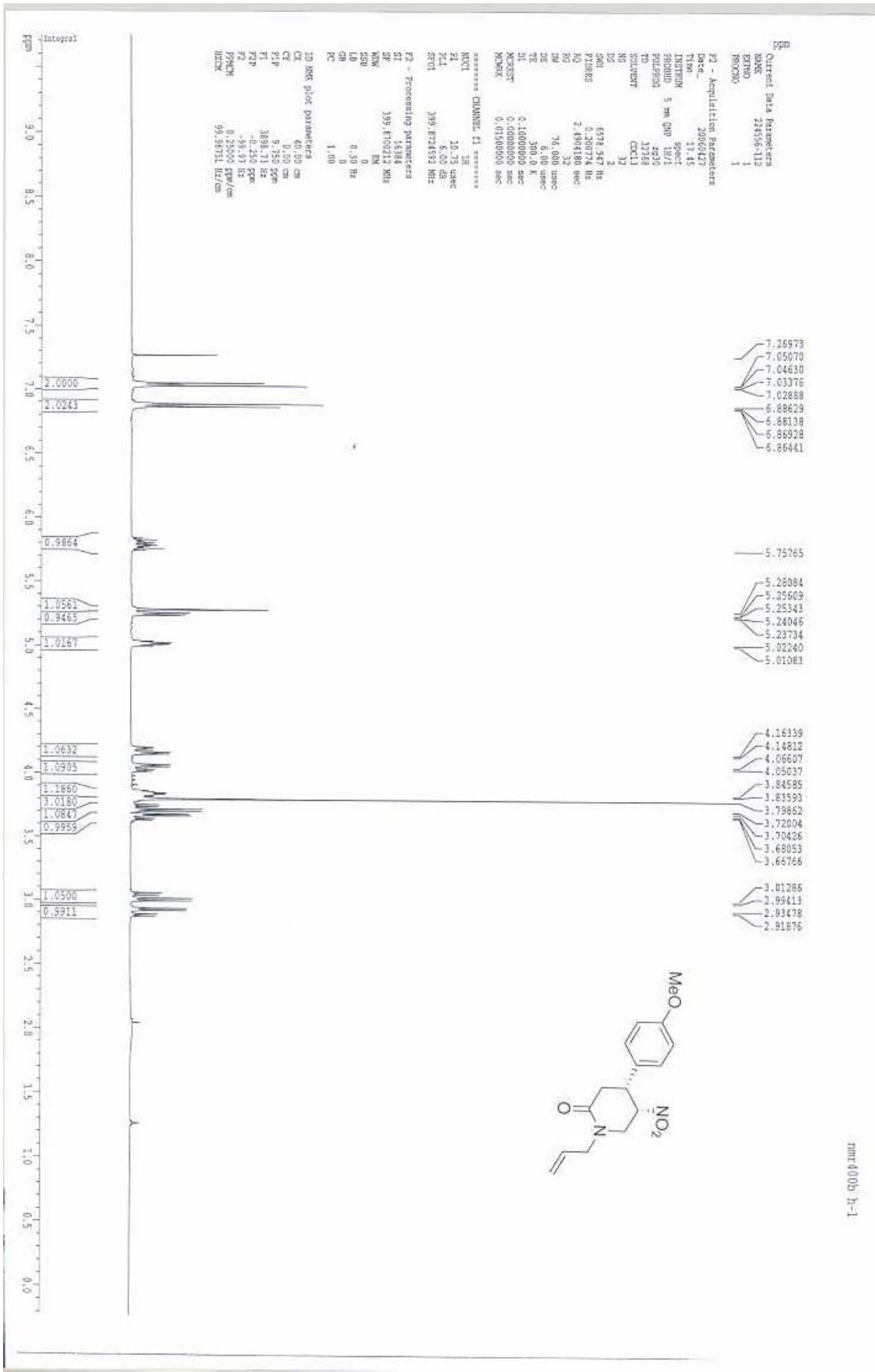




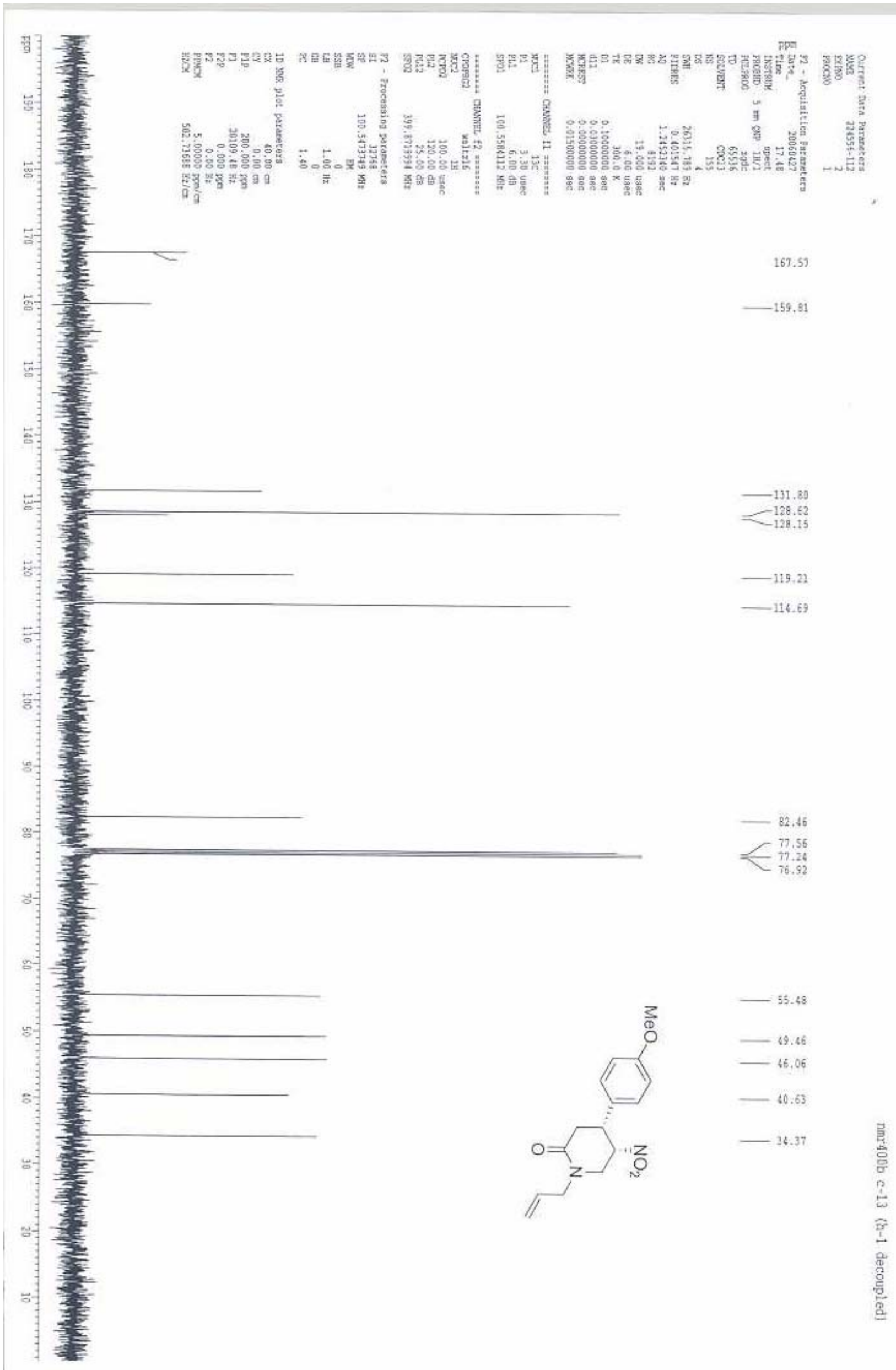




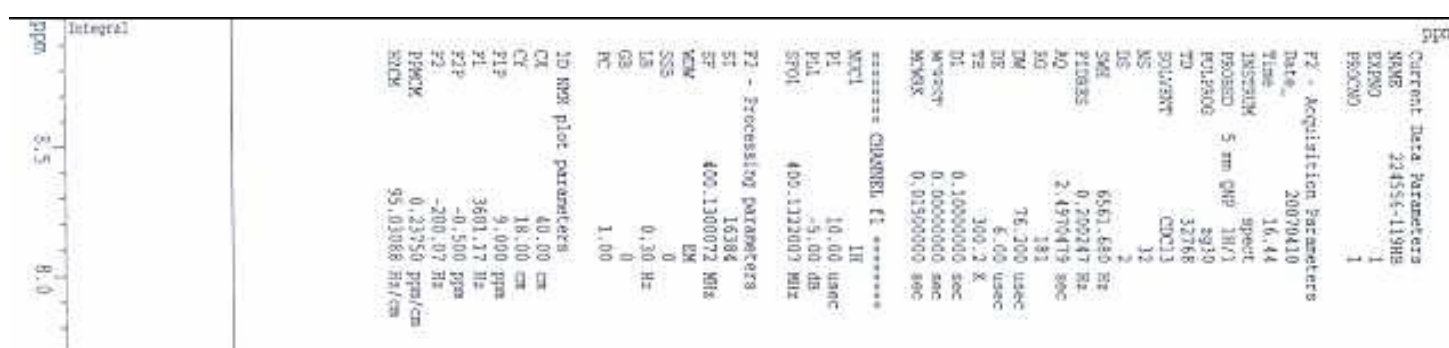

a
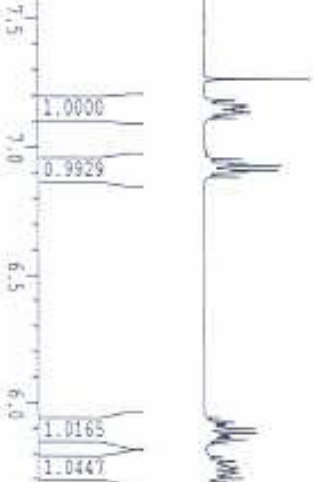

in 1.0560

1.0597

2.0294

in-

?

$-1$

$i \frac{1}{1.0868}$

$\frac{-1.0066}{1.0568}$

51.0614

1.0865

$-\frac{1.1895}{\frac{2.9805}{1.2055}=}$

1.2035

1.0657

$\frac{-1.0825}{-\frac{1.0254}{1.0965}}$ 1.040

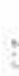

-

$=$

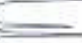

트
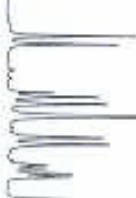

$3=$

$=$
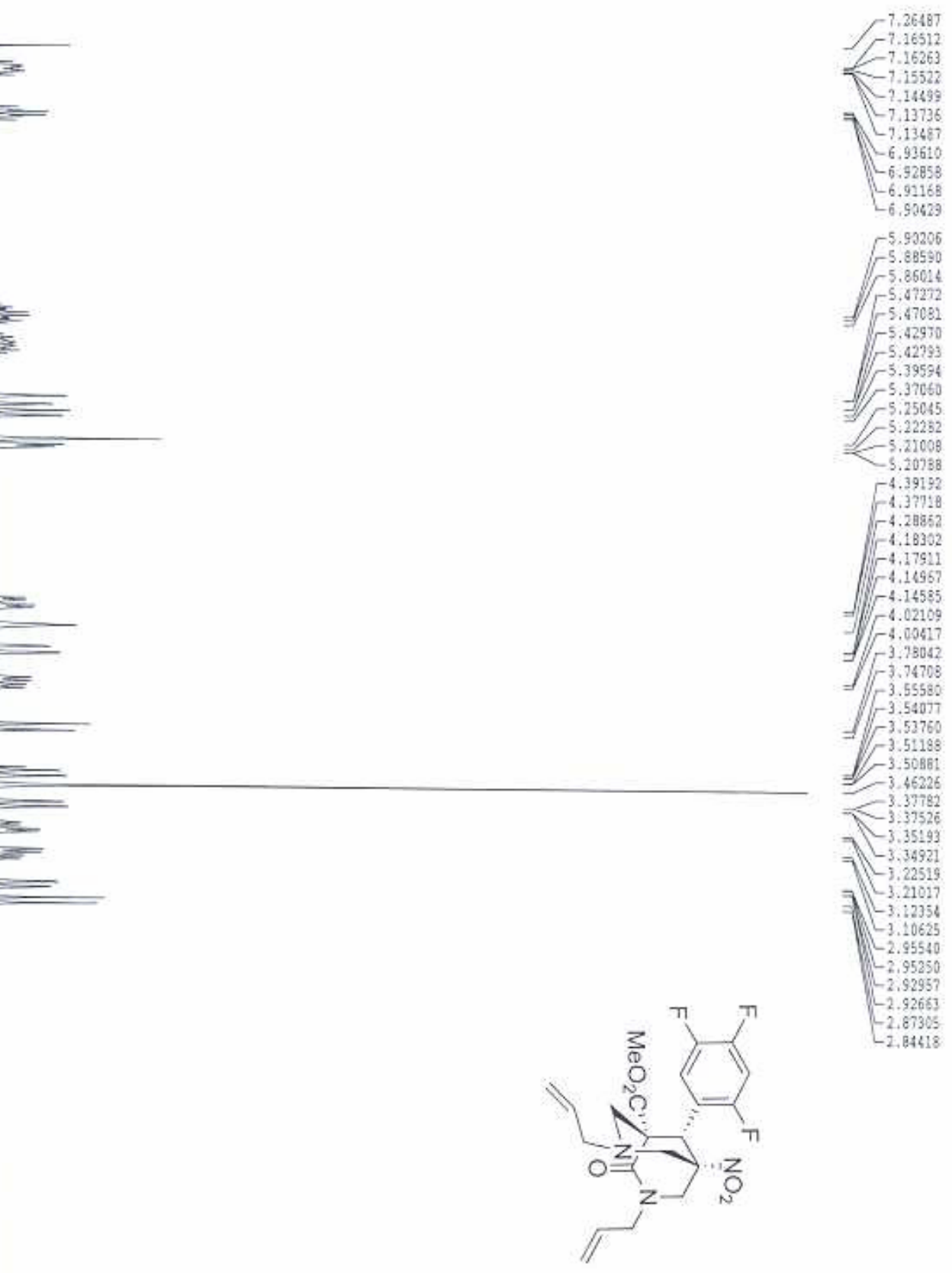

a-

in

5

in

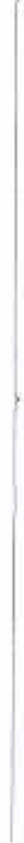




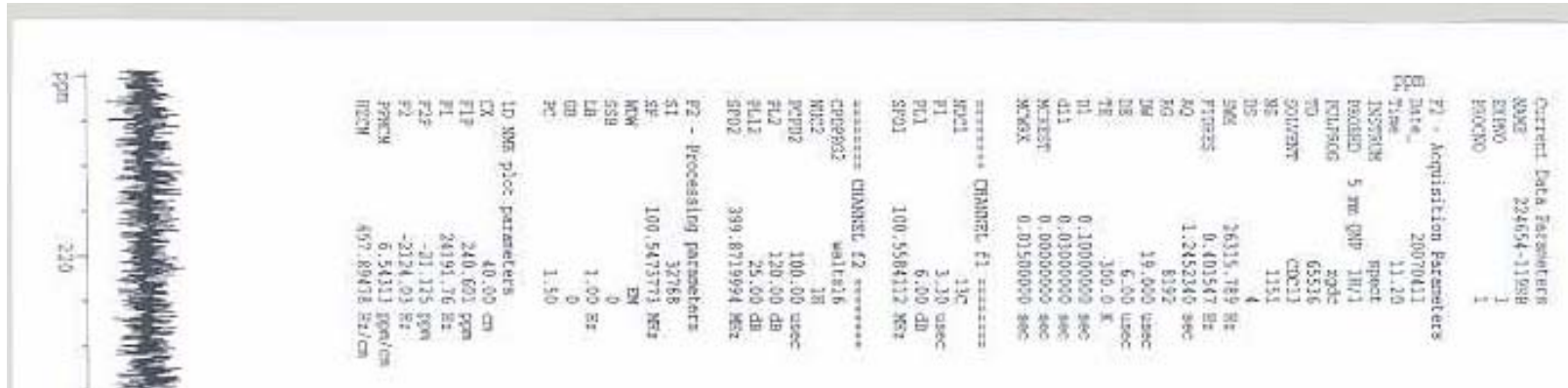

항

봉-

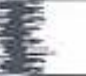

$-168.10$

-158.06

$-157,9$

$\int_{-155}^{-155.62}$

$-151.60$

$-151.26$

7. -146.73

$-148.25$

$-145.68$

흠

.

:

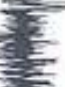

${ }^{120.67}$

$-18.98$

$\sim_{-112}^{-118}$

$-117.49$

$-11728$

106.03
-125.93

탕-

$-105.73$

sg-
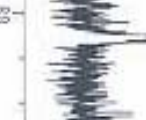

$-36.29$

C 77.54
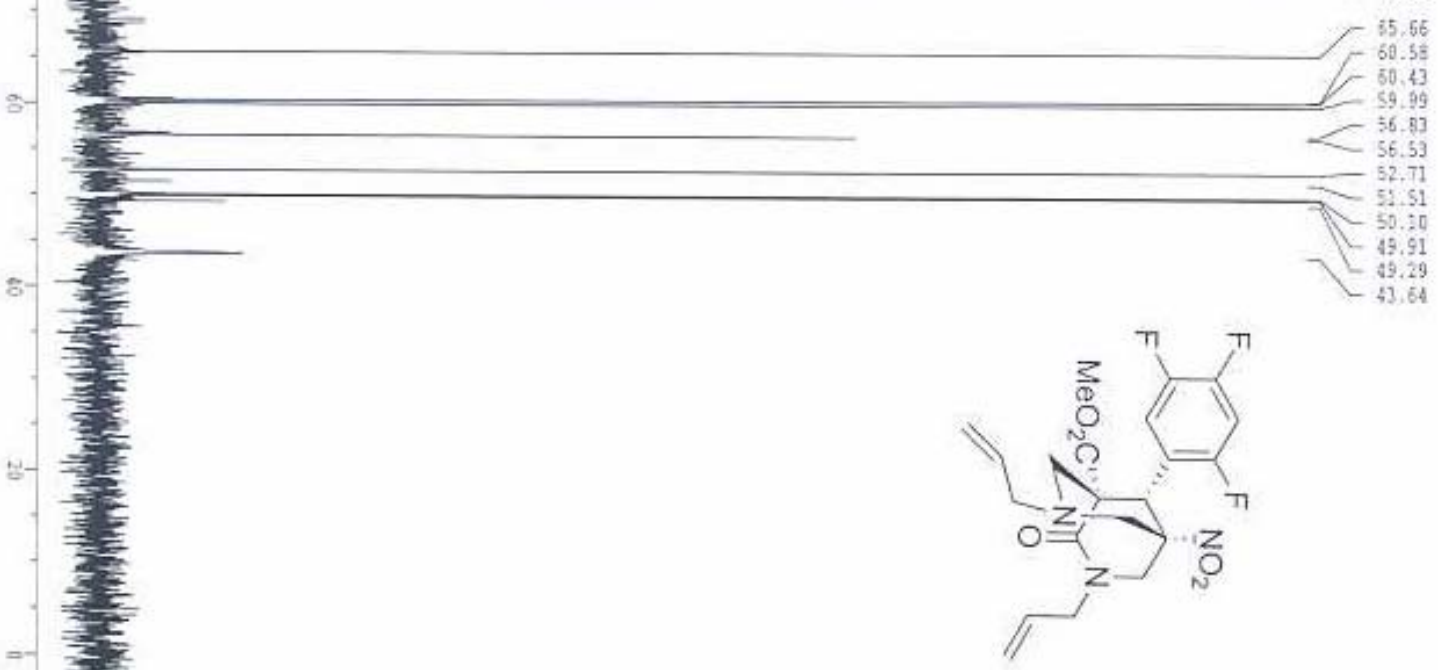

43.64

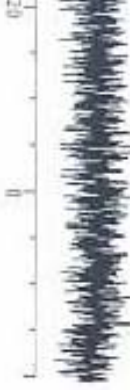




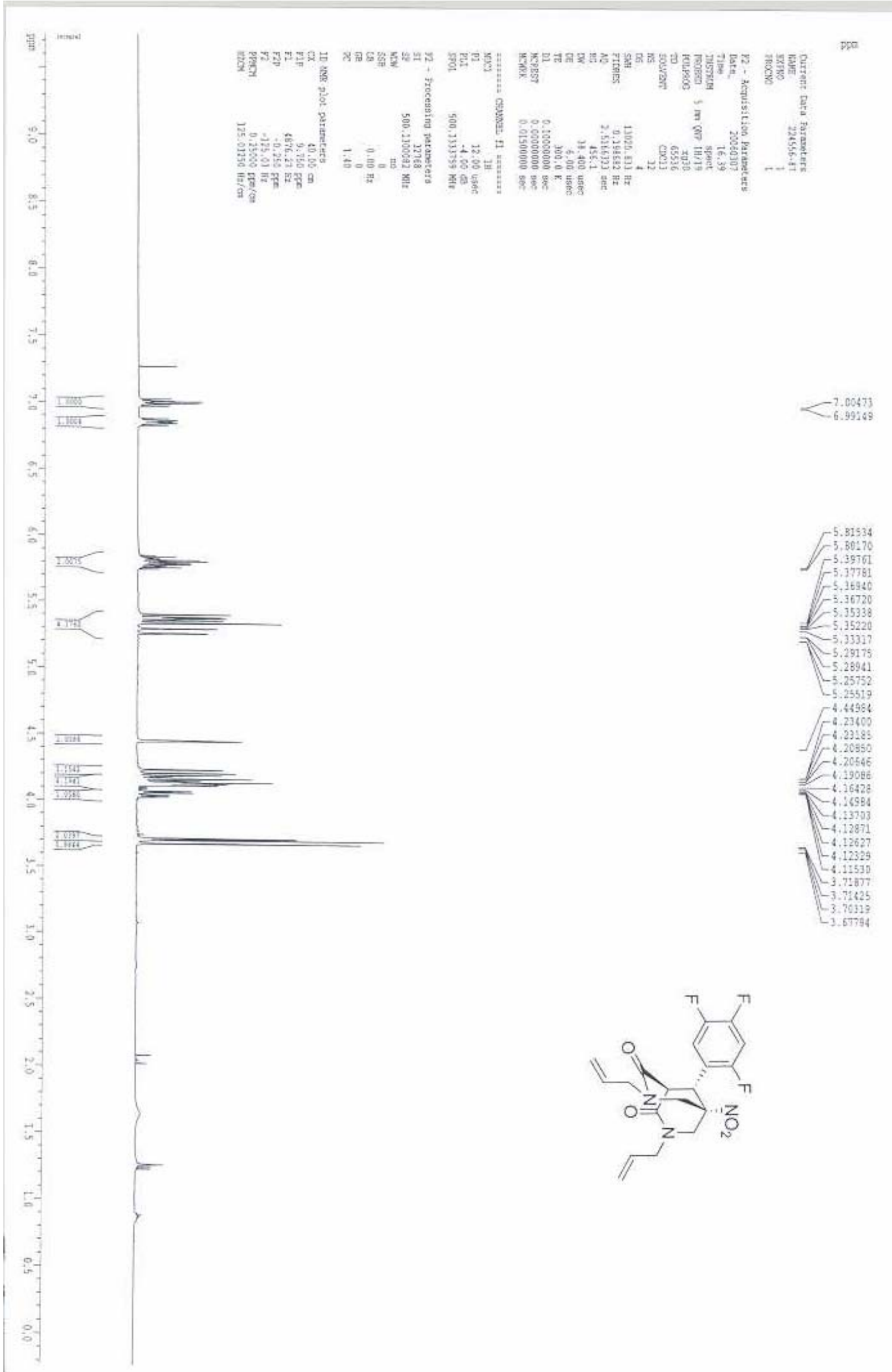




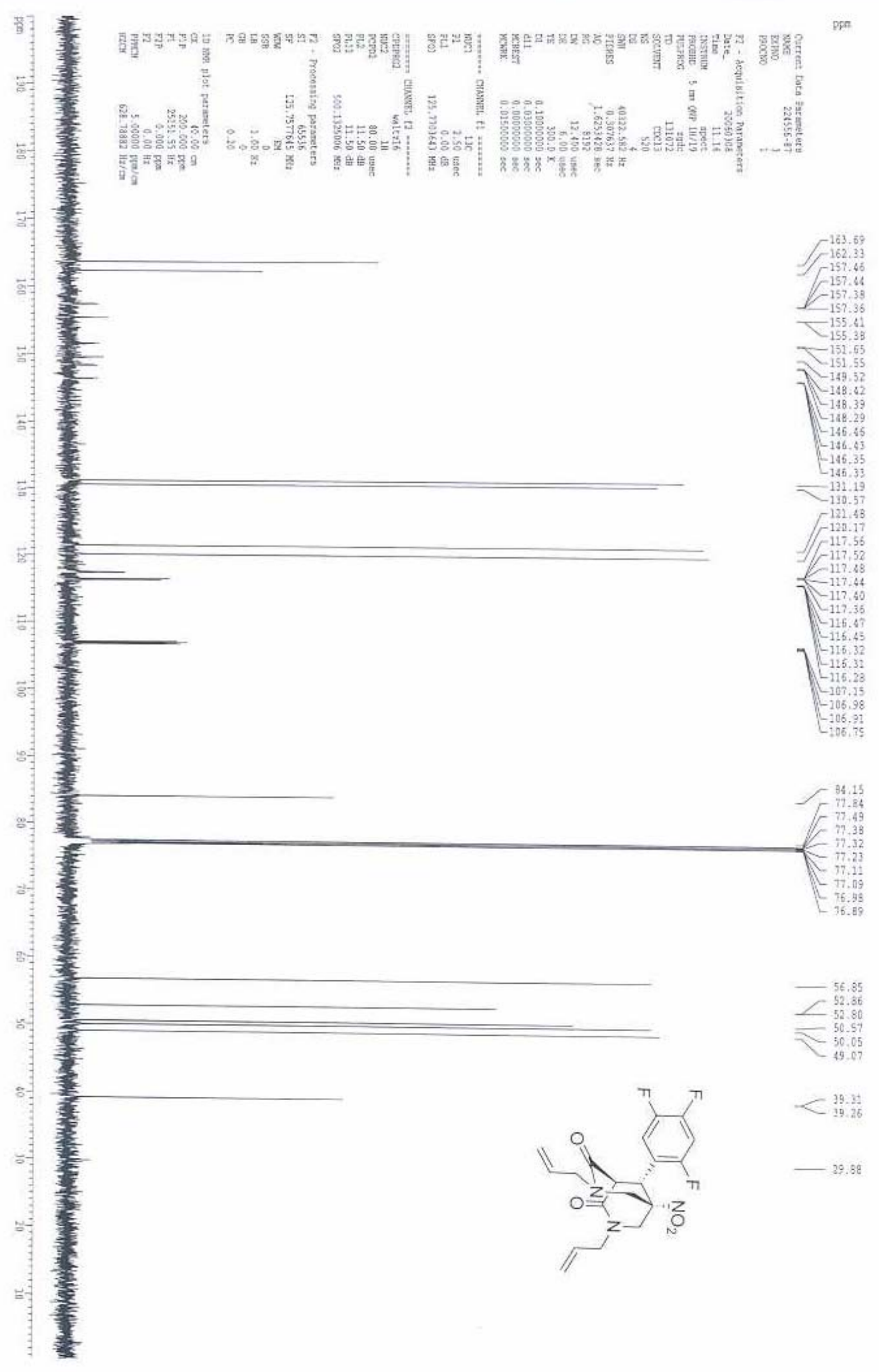




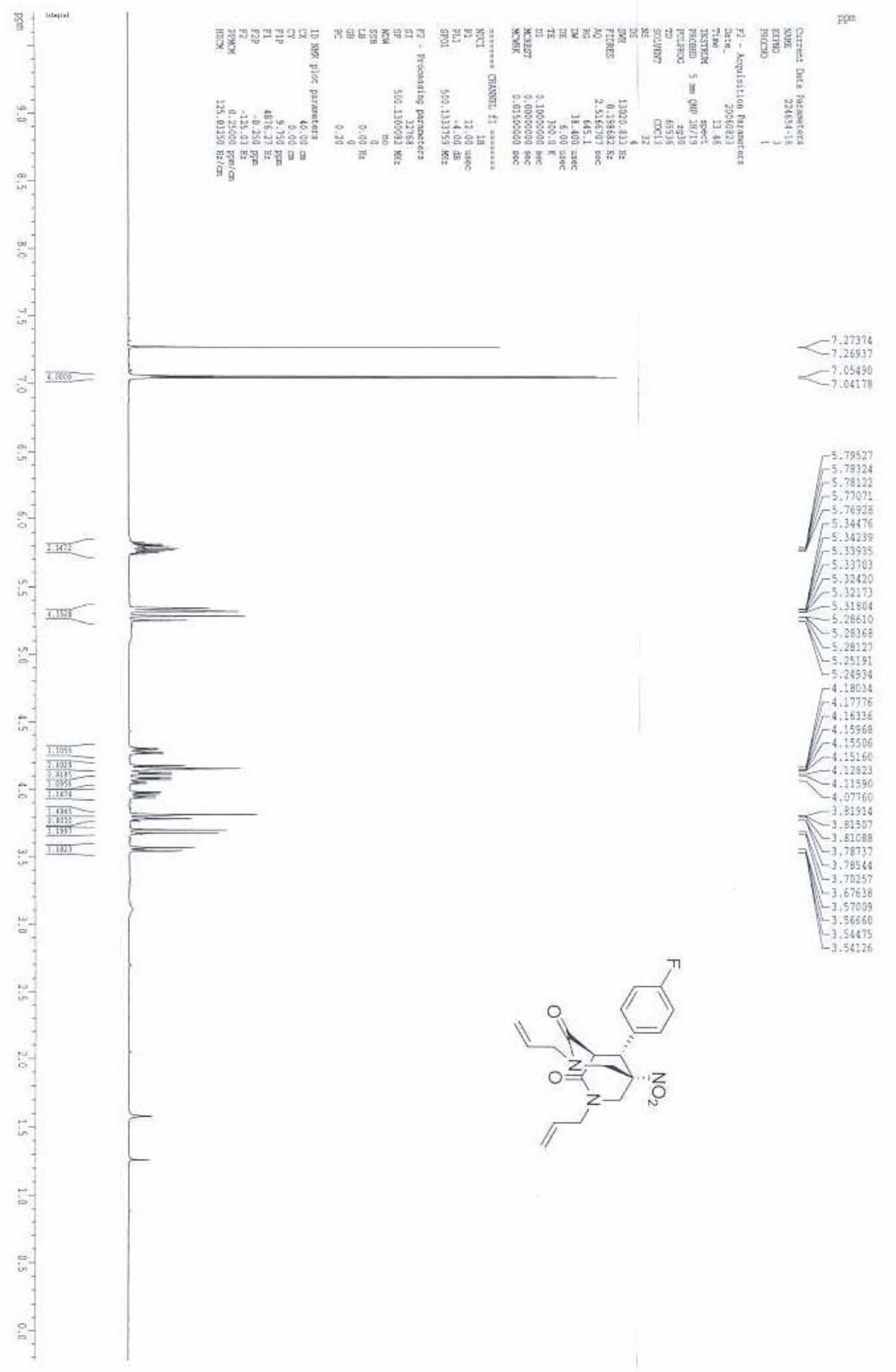




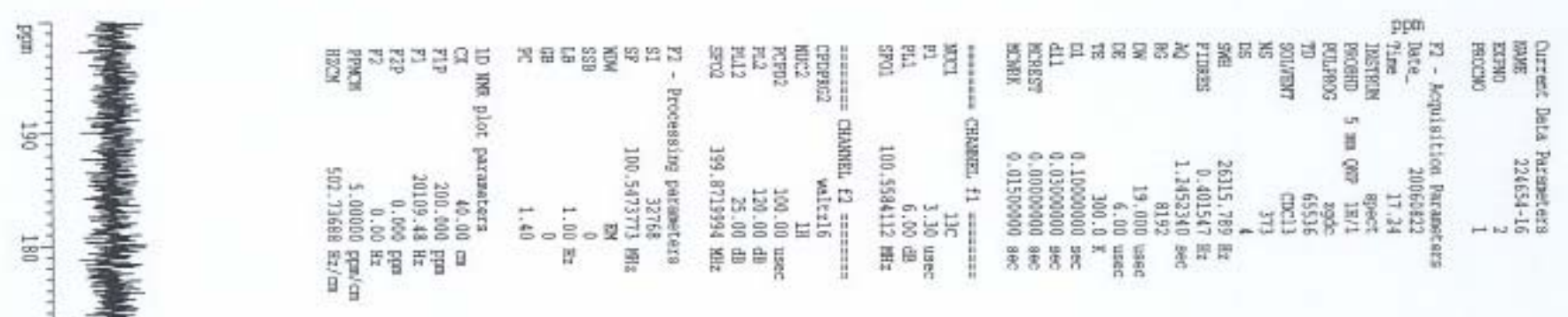

덩-

함

퐁

형

항

녕

텽

통

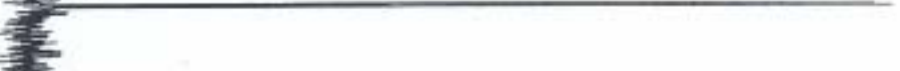

164.54
$\sim_{164.43}^{16.43}$
$\mathbf{r}_{-161.95}$

$\int_{131.30}^{133}$

$-129.57$

129.59
-129.22

${ }_{-129.19}^{-129.22}$

$-120.89$

$-119.78$

$-116.85$

116.63

85.37

罗-

27.54

$-77.23$

73.84

공

8
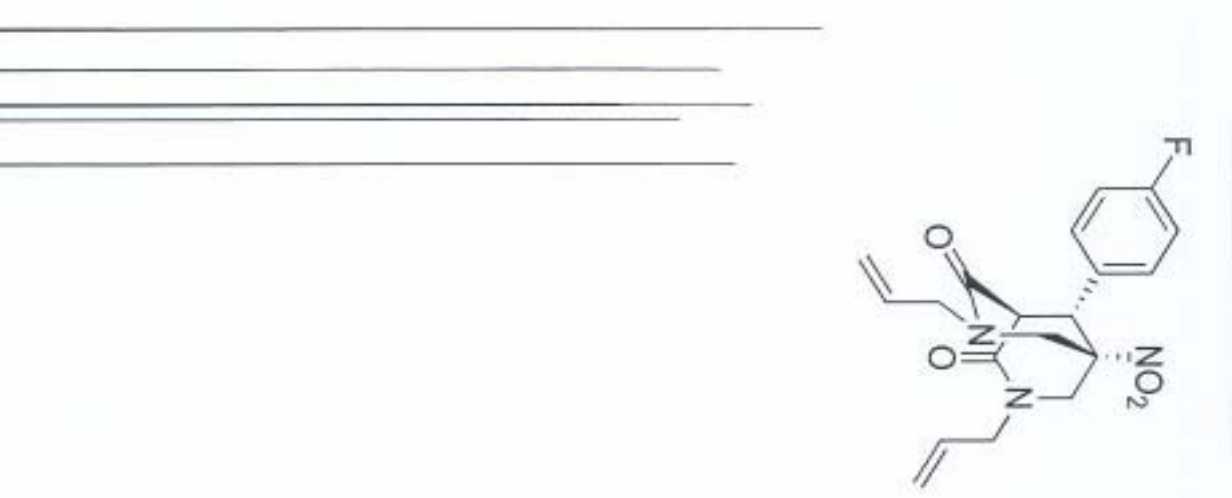

$-56.07$

-52.85
-

$\begin{array}{r}50.07 \\ \hline\end{array}$

-48.78
-

京

궁

극 


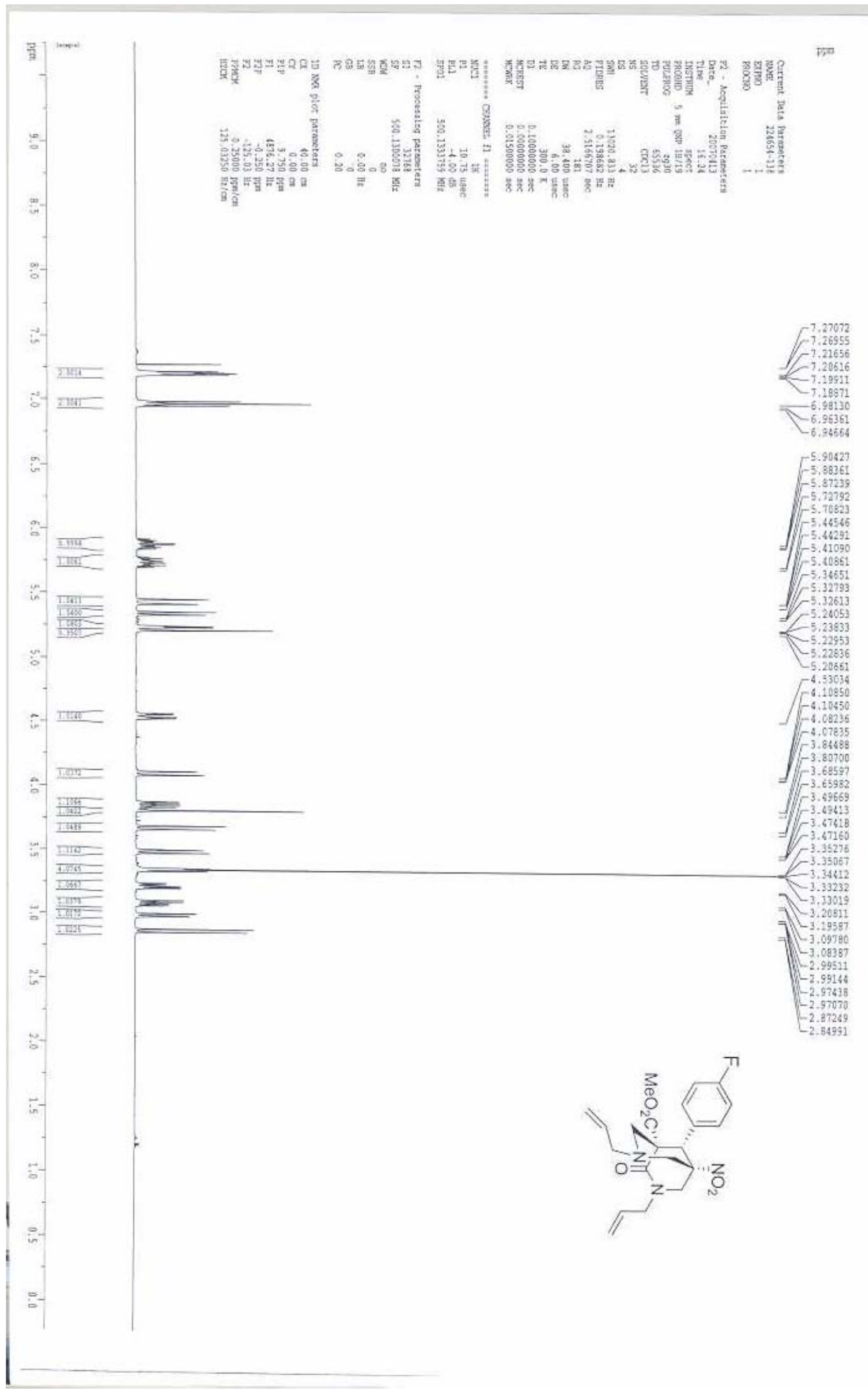




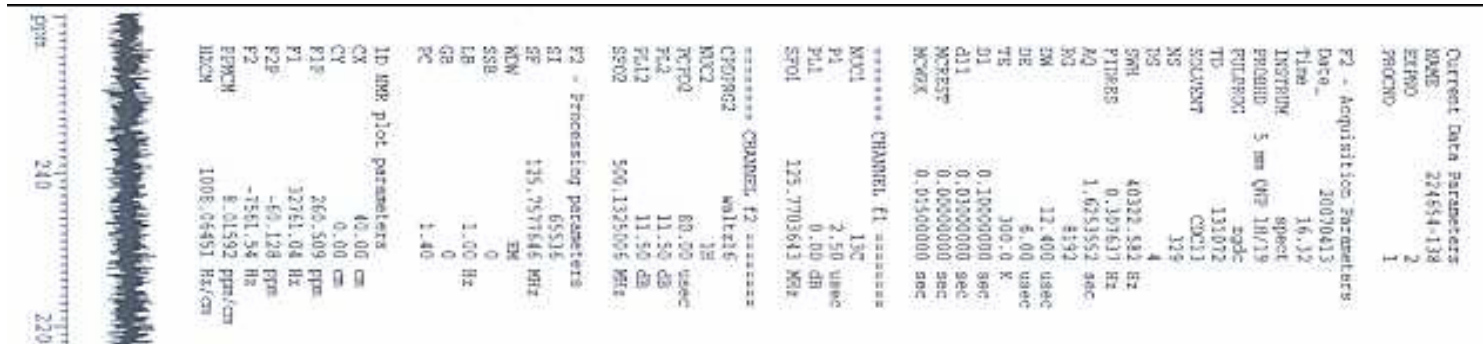

,

猔素
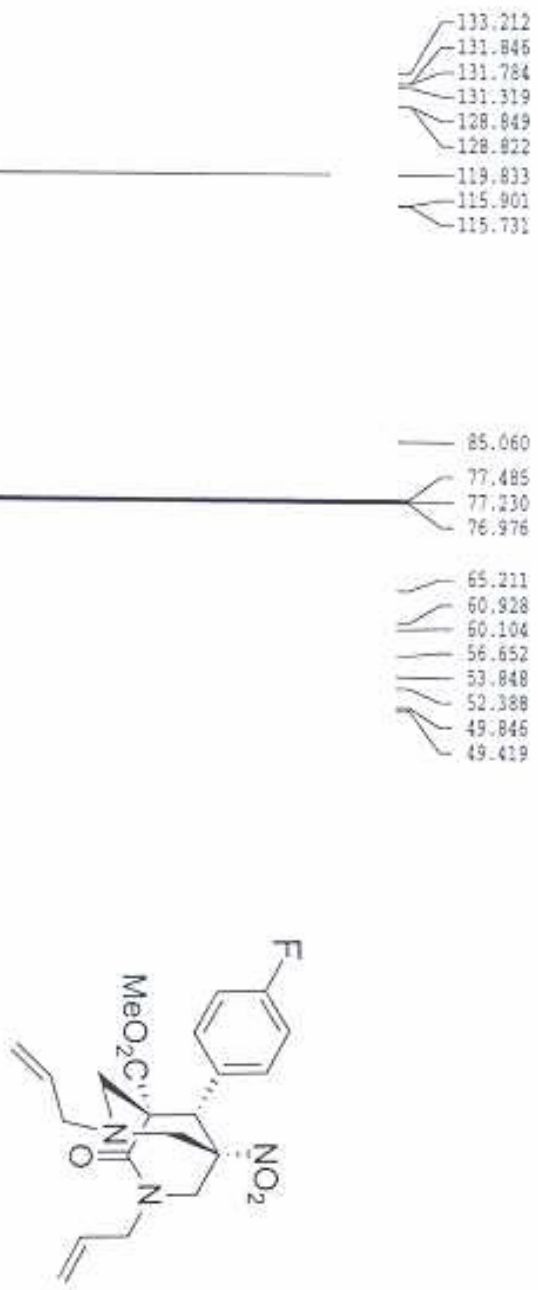

- 85.060 $-77.485$ - 76.276 $-65.211$ - 60.928 - 60.108 - 56.652 -33.848
$-\quad 52.389$ $\longrightarrow 49.845$ 


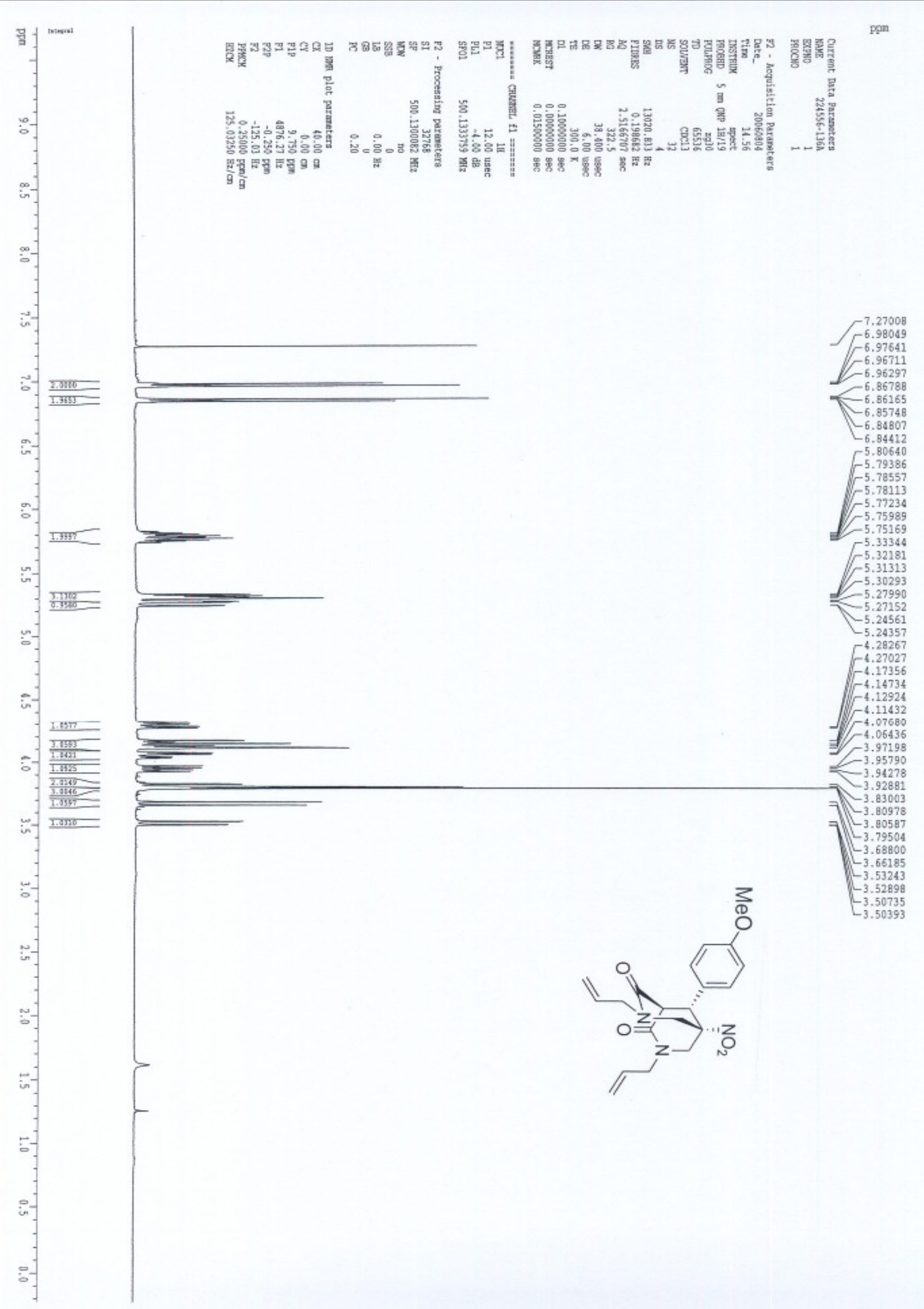




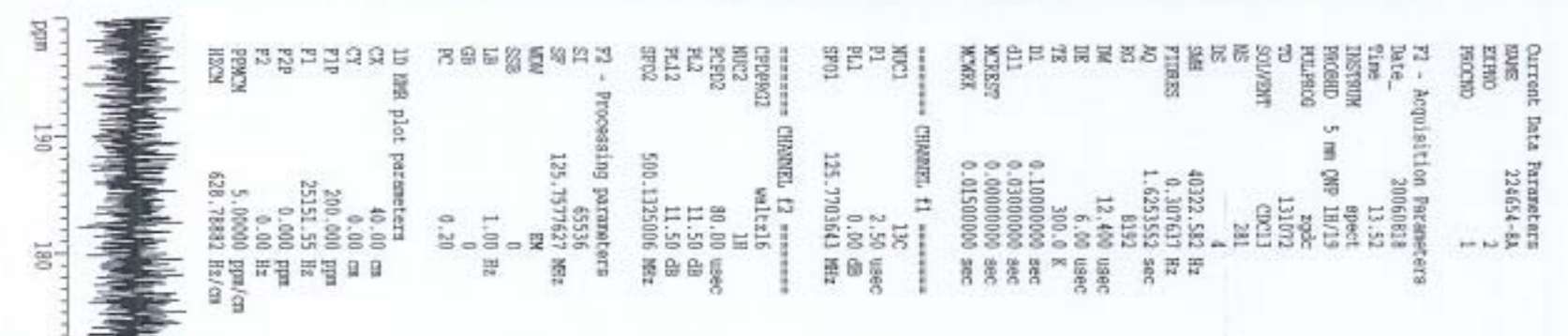

병

형

항.

碀

낭-

는

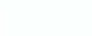

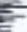

s.

$-164.93$

$<-163.48$

$-150.31$

난

53.

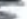

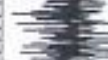

=

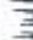

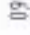

.

更 ,

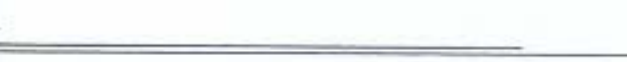

注

桨

政。

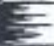

- 77.09

- 76.98

$\begin{array}{r}76.90 \\ -76.89 \\ \hline\end{array}$

글
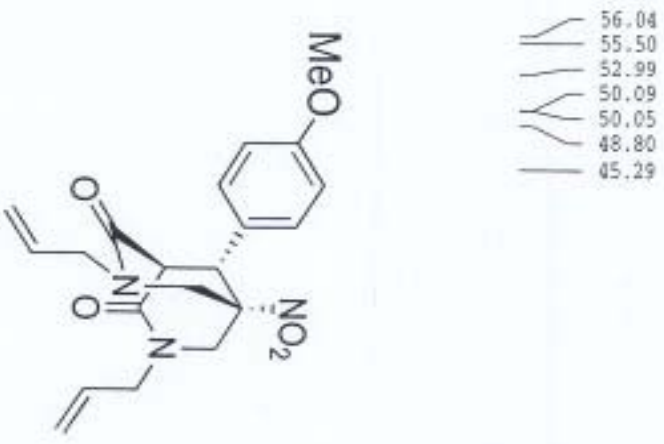


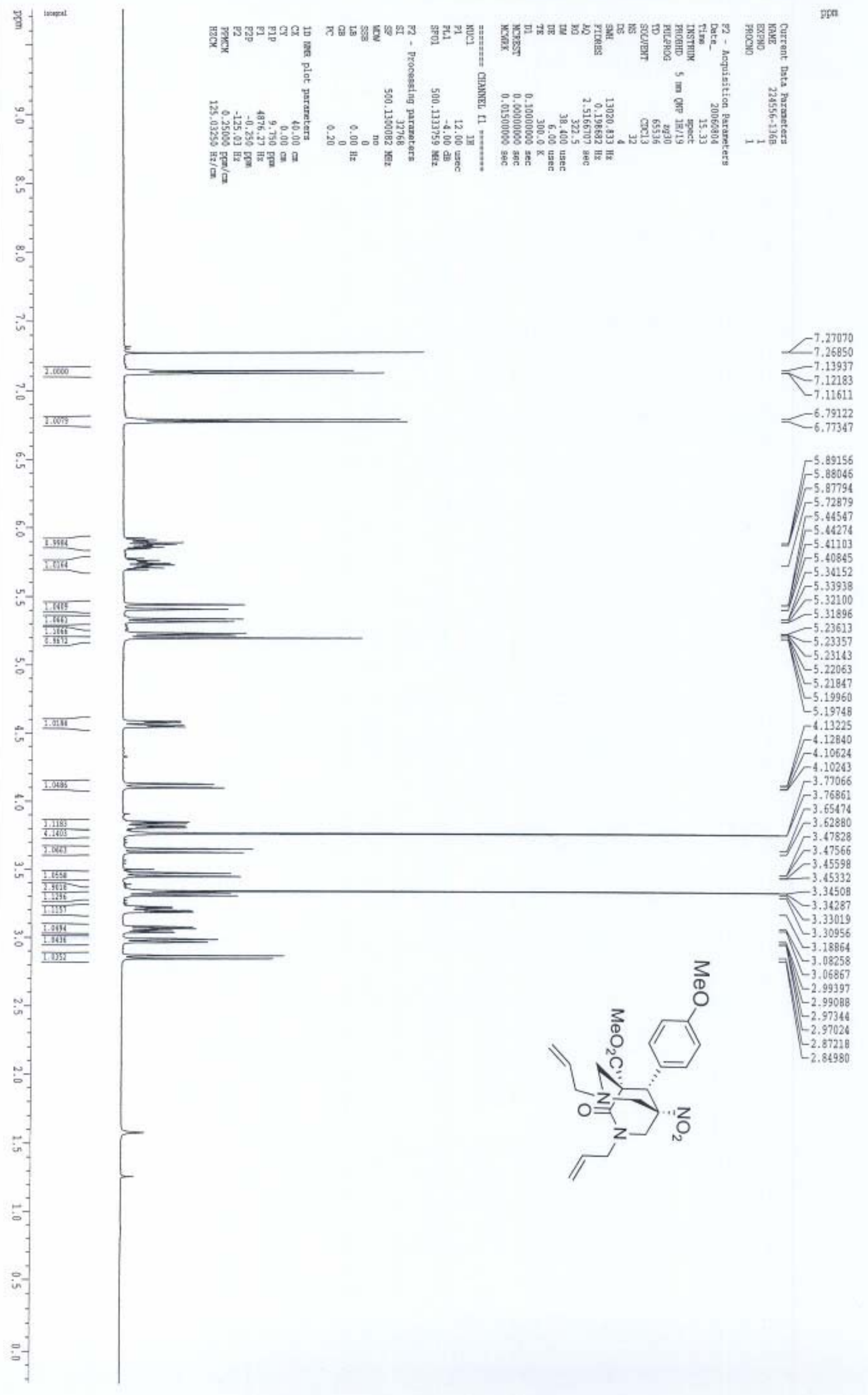




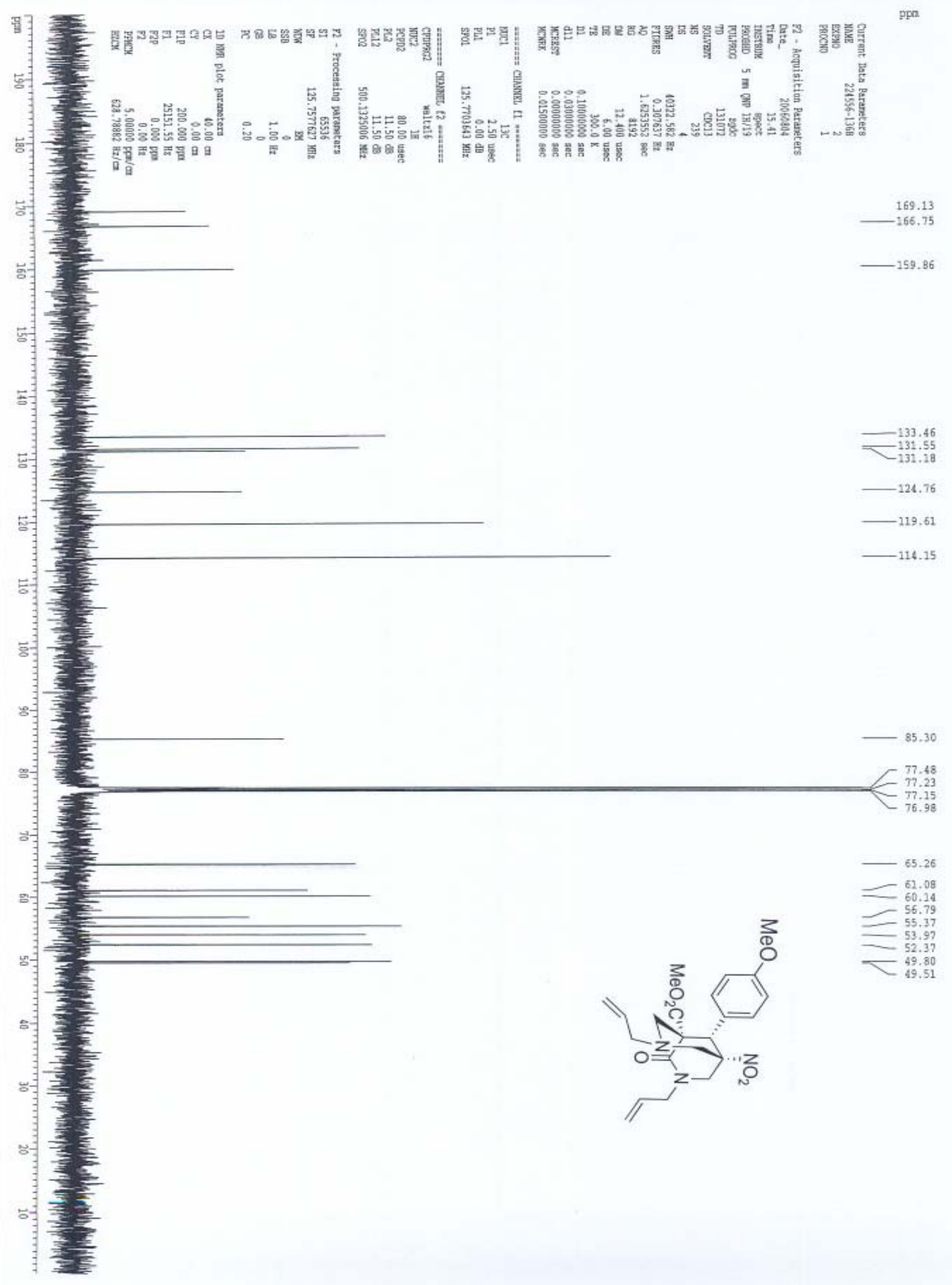




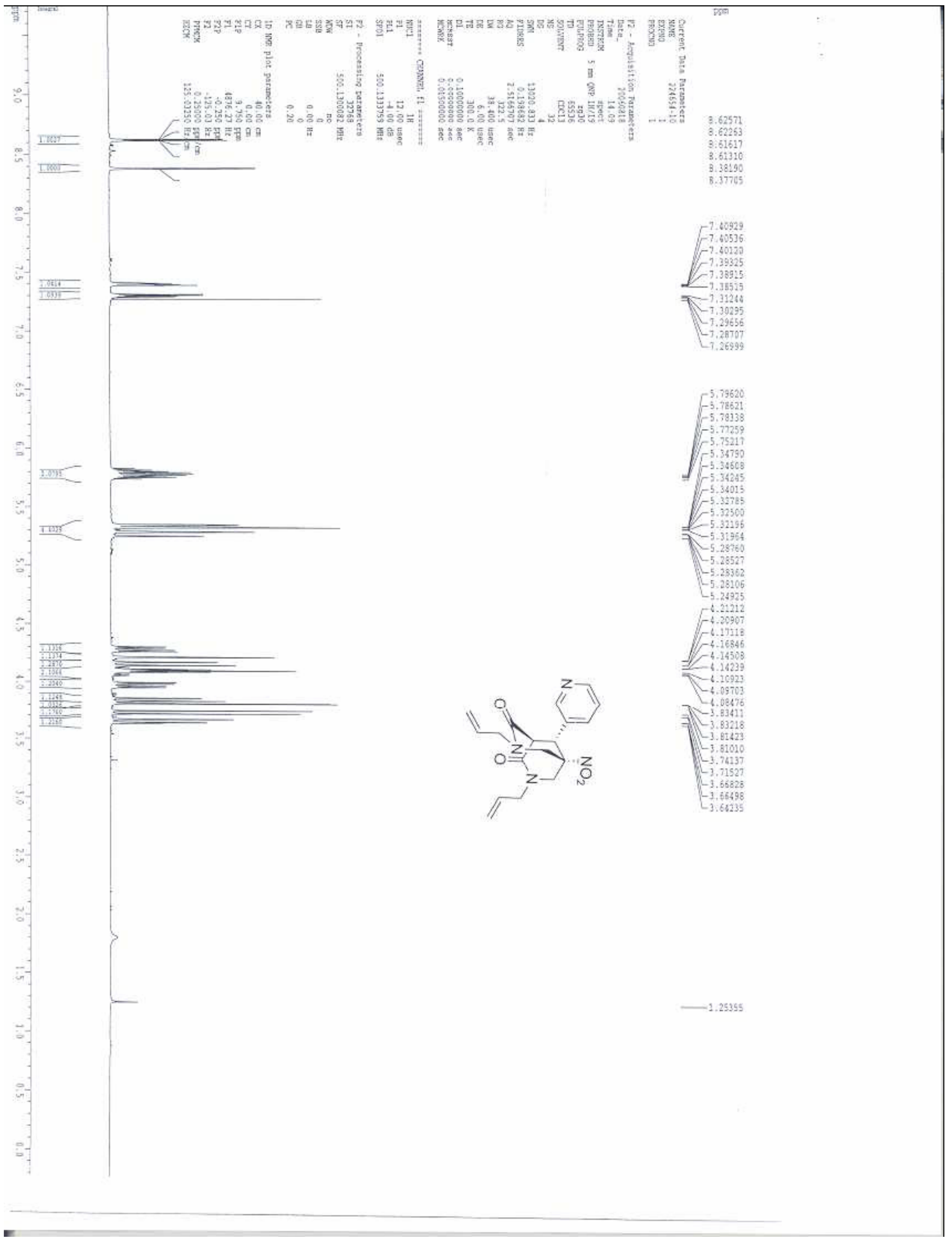




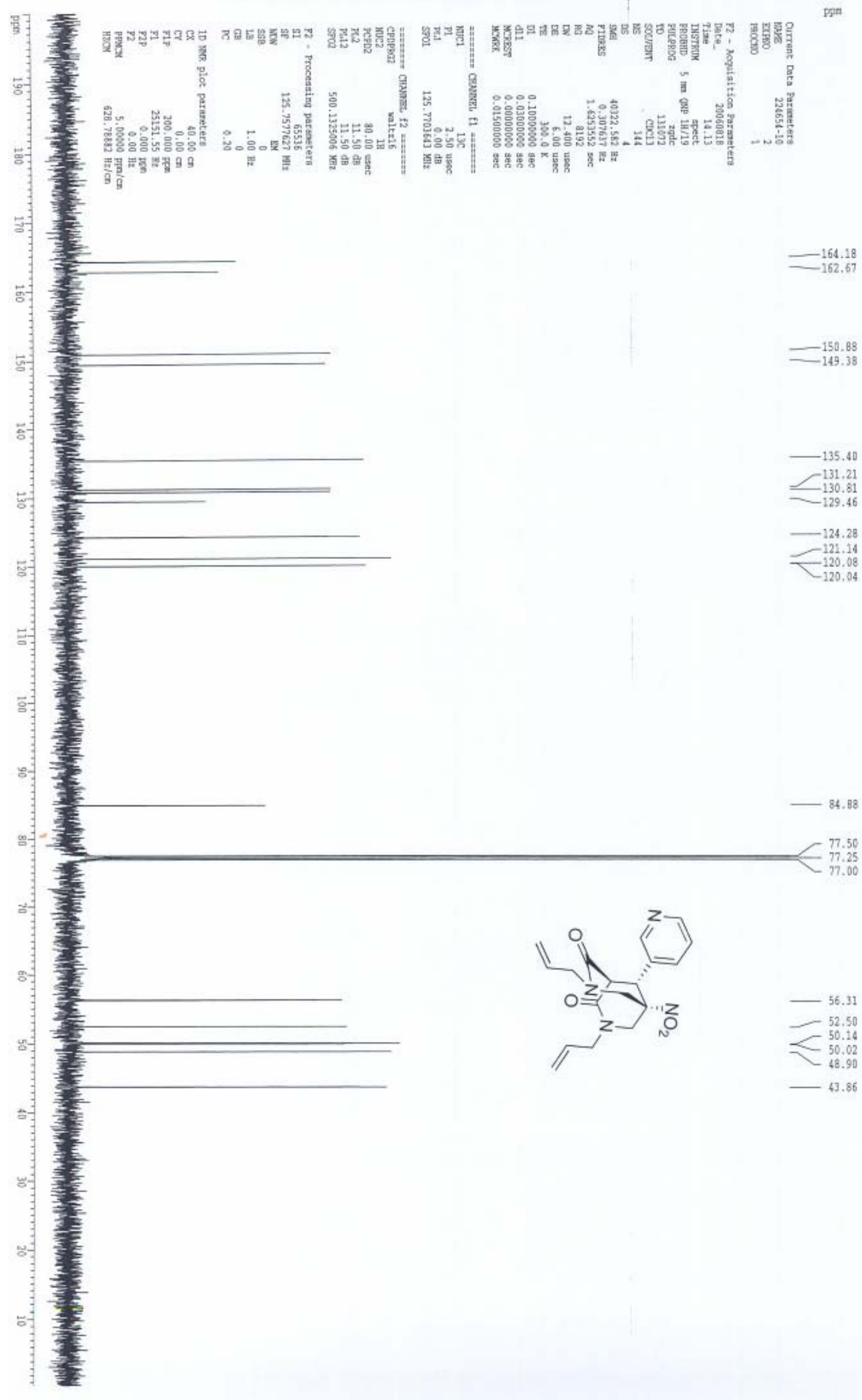




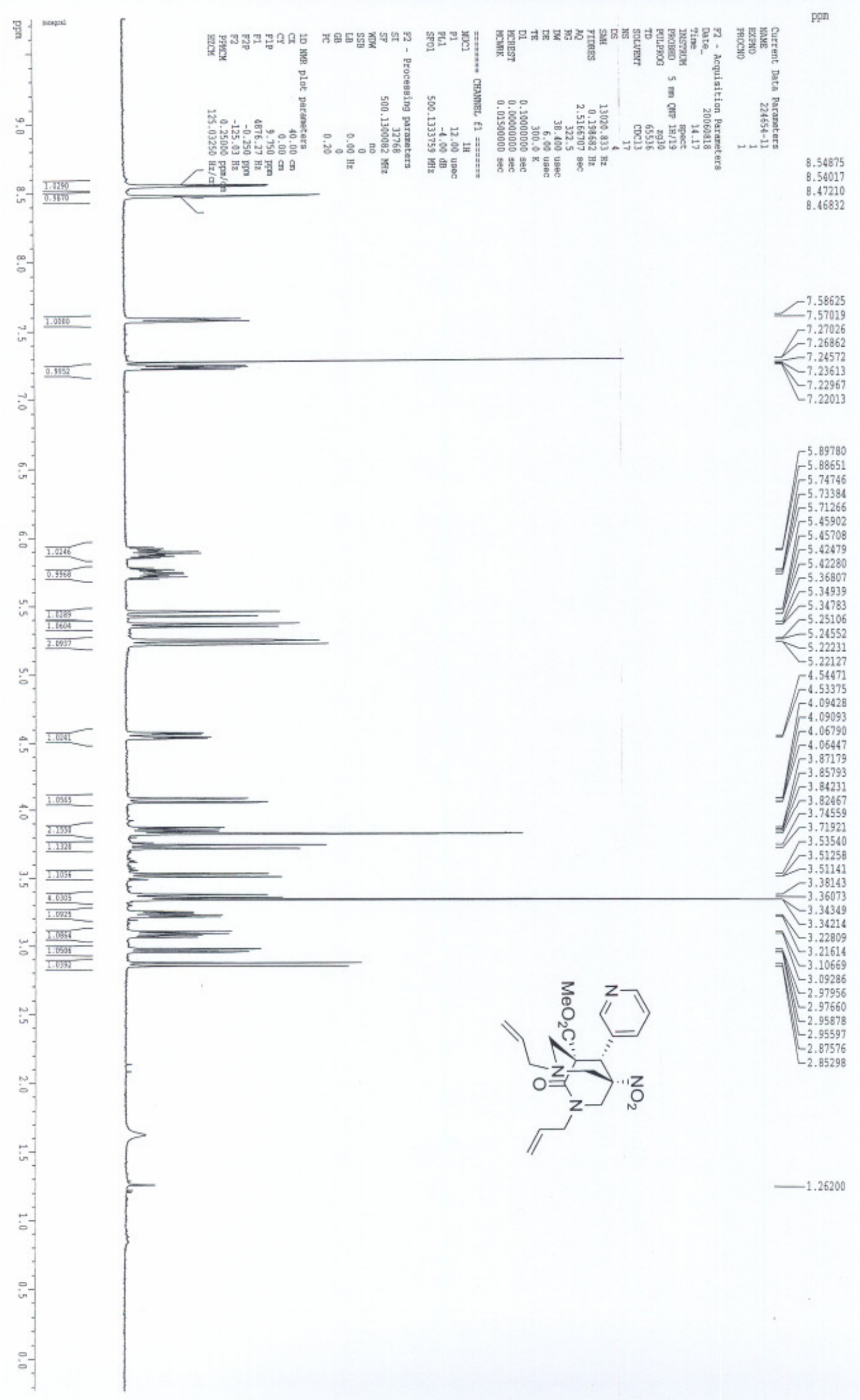




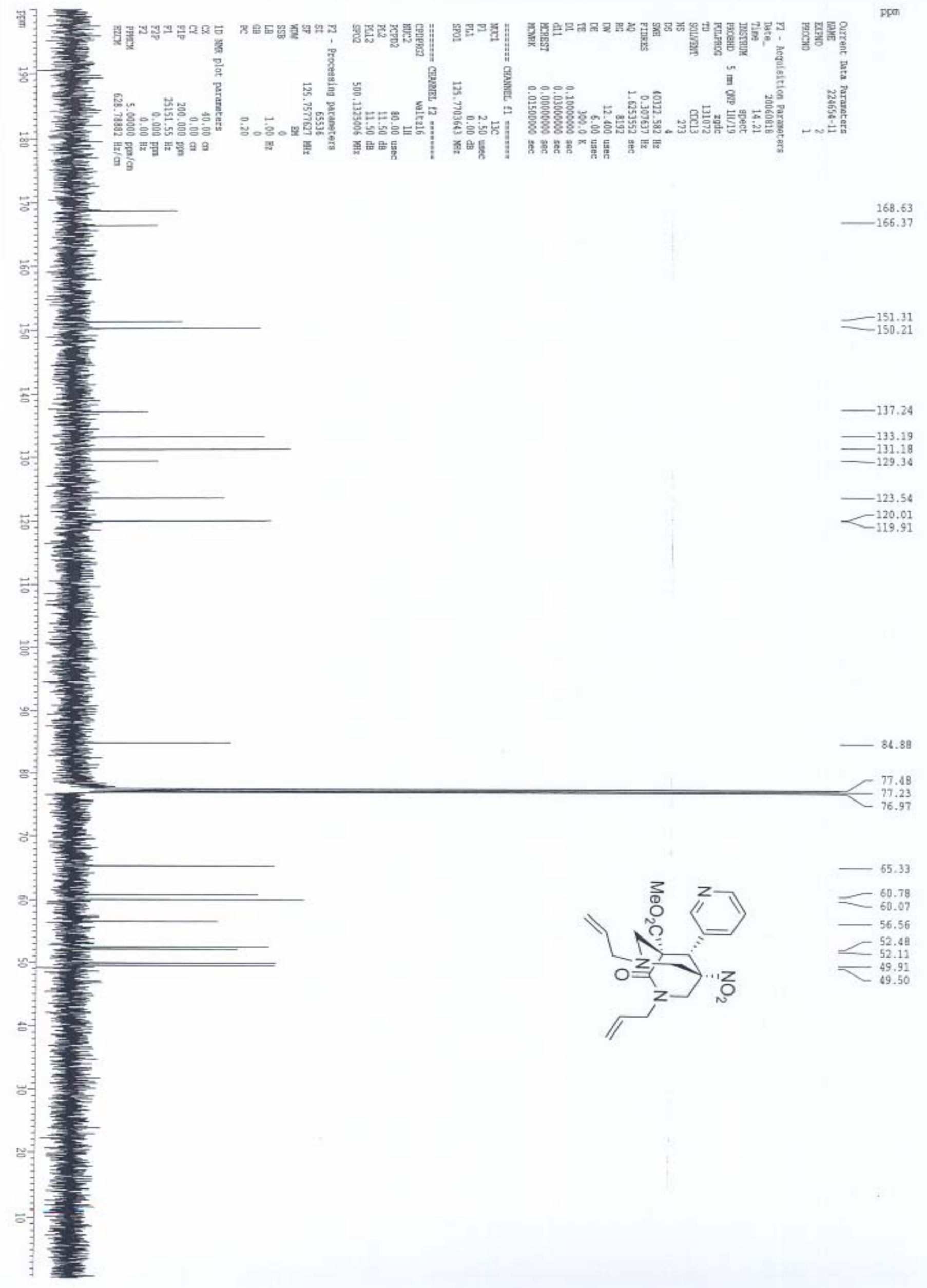




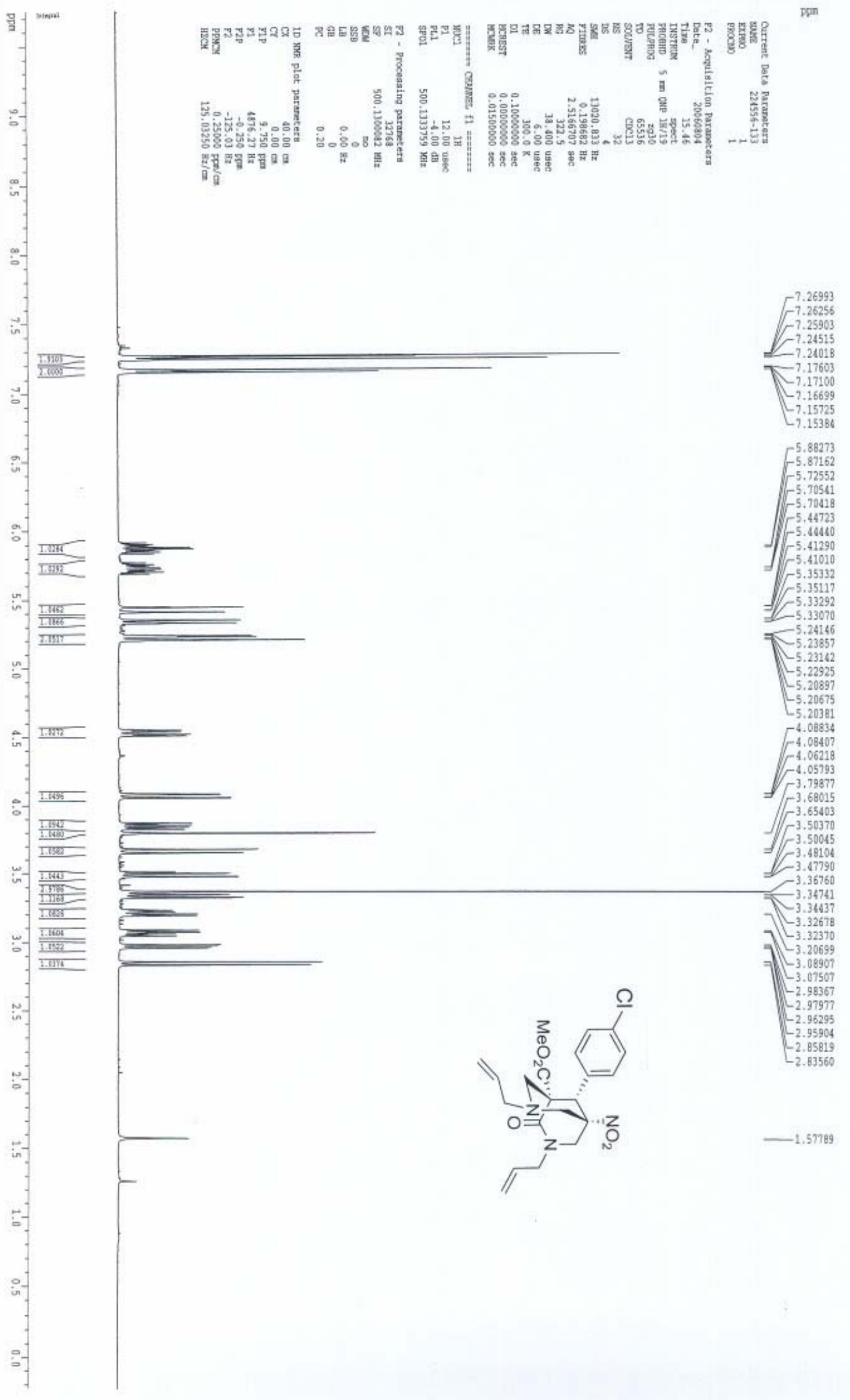




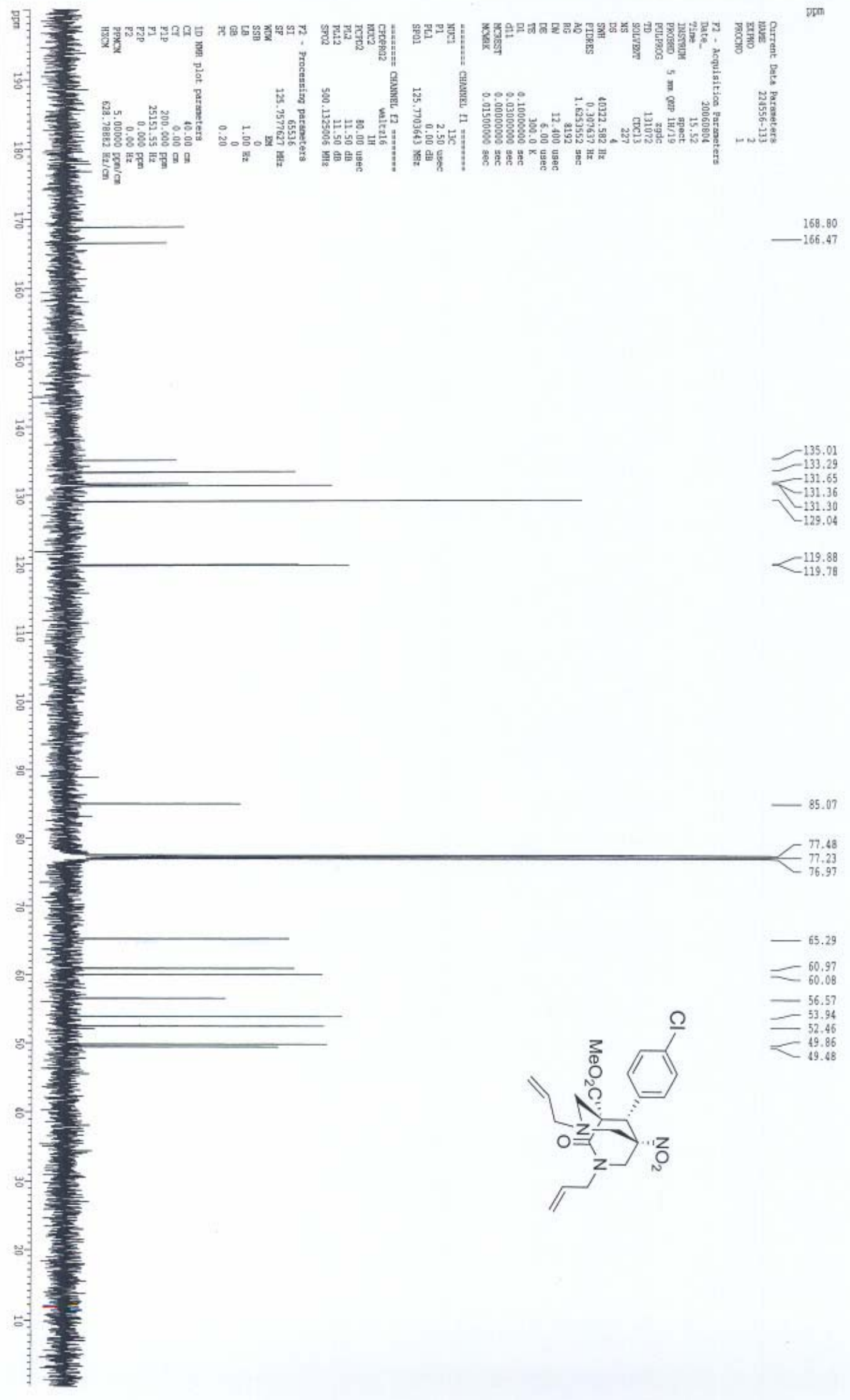




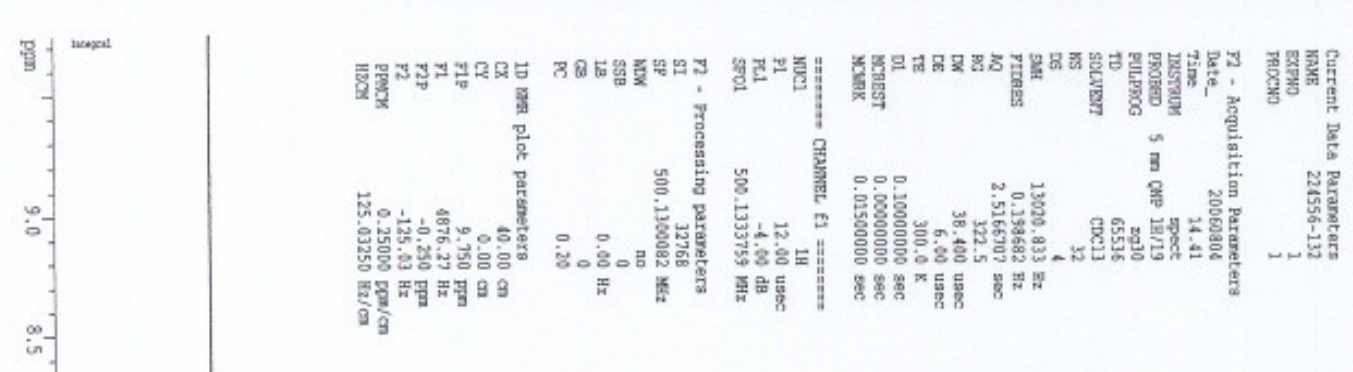

ppa.

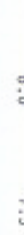

2.000

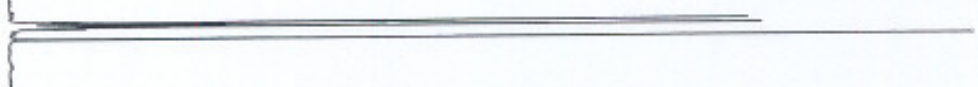

$\because$

2.091

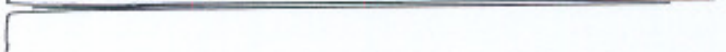

प.05

in

द.5211

0

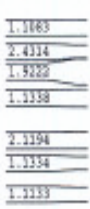

जn
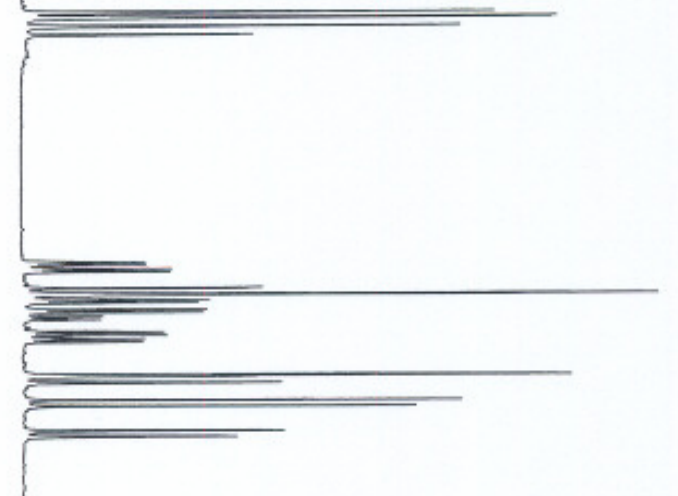

$-$

-

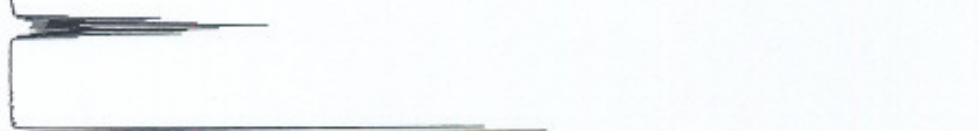

7.34311
-7.33758
-7.33350
-7.32067
-7.32042

$-7.32042$

$-7.25995$

$-7.01035$

-7.00488
-7.00103
$-6.9916 ?$

$-6.98779$

$\Gamma^{5.80992}$

-5.77648
-5.76358
-5.75552

$\sqrt{-5.75552}$

${ }^{-5.74301}$

5.33857

${ }_{-5.33642}^{5.33857}$

$-5.31831$

$\mathbb{V}_{-5.31336}^{5.31583}$

5.28191
-5.27902
-5.2405

$-5.24796$

ᄂ 5.24549

5.25706
-4.2454

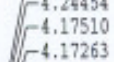

$\Gamma^{4.14882}$

$F_{-4.12447}^{-1.12822}$

$-4.11224$

4.08314
-4.07063

$-3.98820$

3.97422

$-3.80910$

$-3.80510$

$-3.80178$

$-3.77615$

$1-3.70256$

hL-3.67536

-3.57346
-3.56997

$-3.54807$

$L_{3.54455}$

in

in-

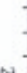

0

-

in.
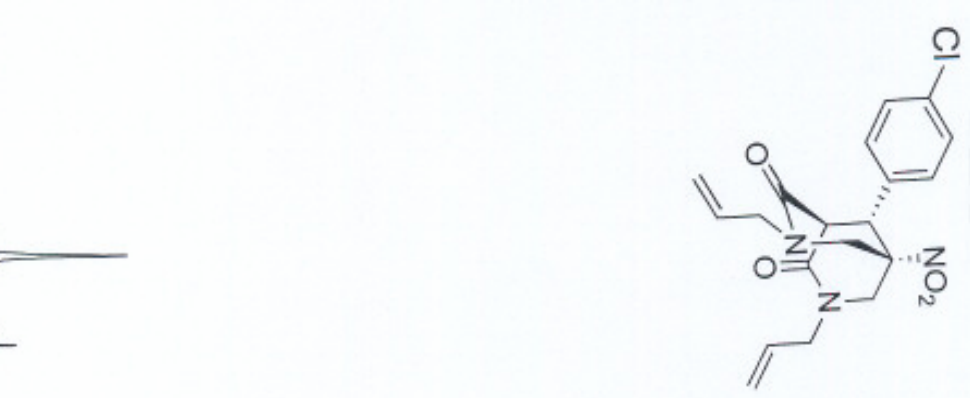

$-1.63845$

$-1.26066$ 


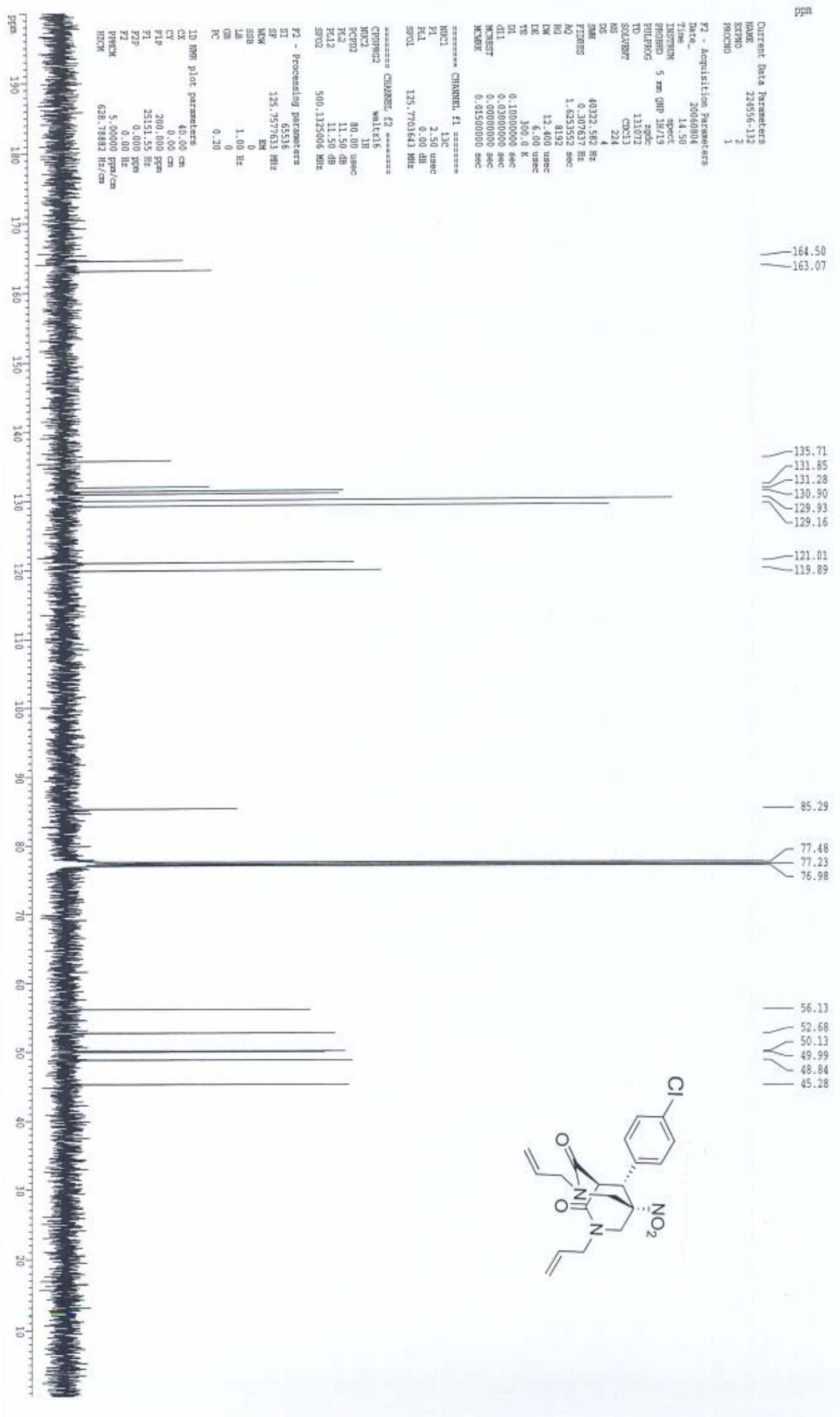




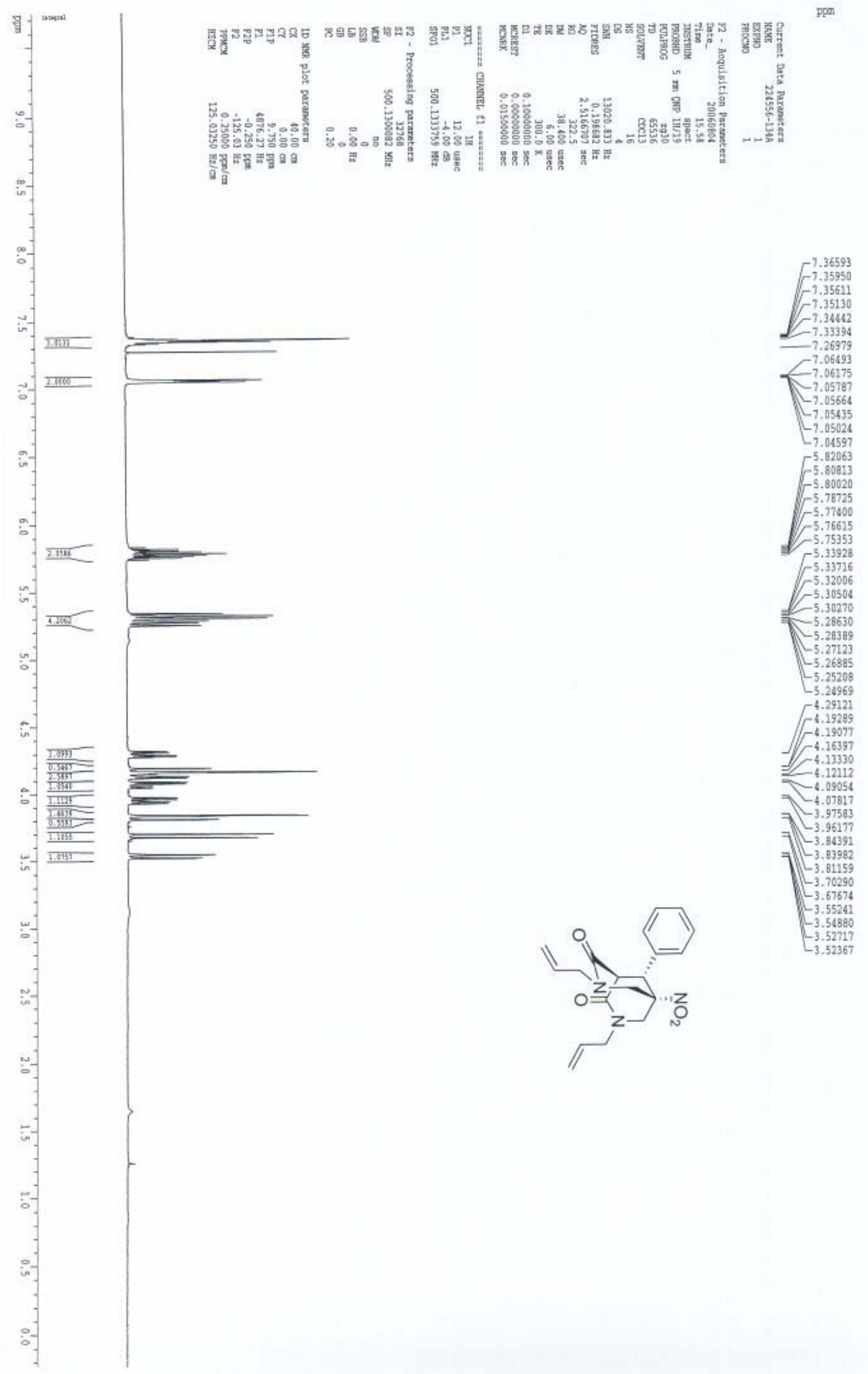




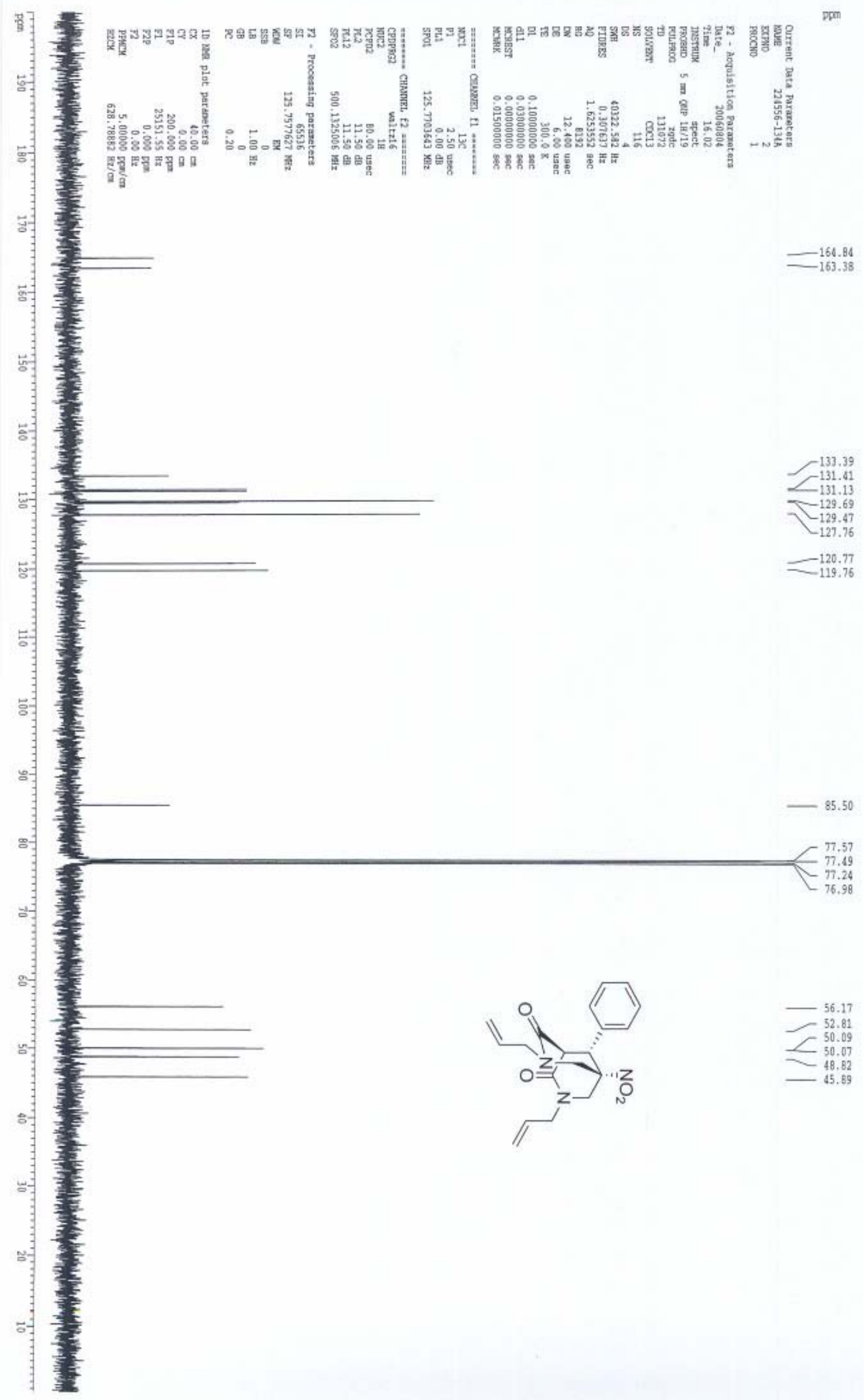




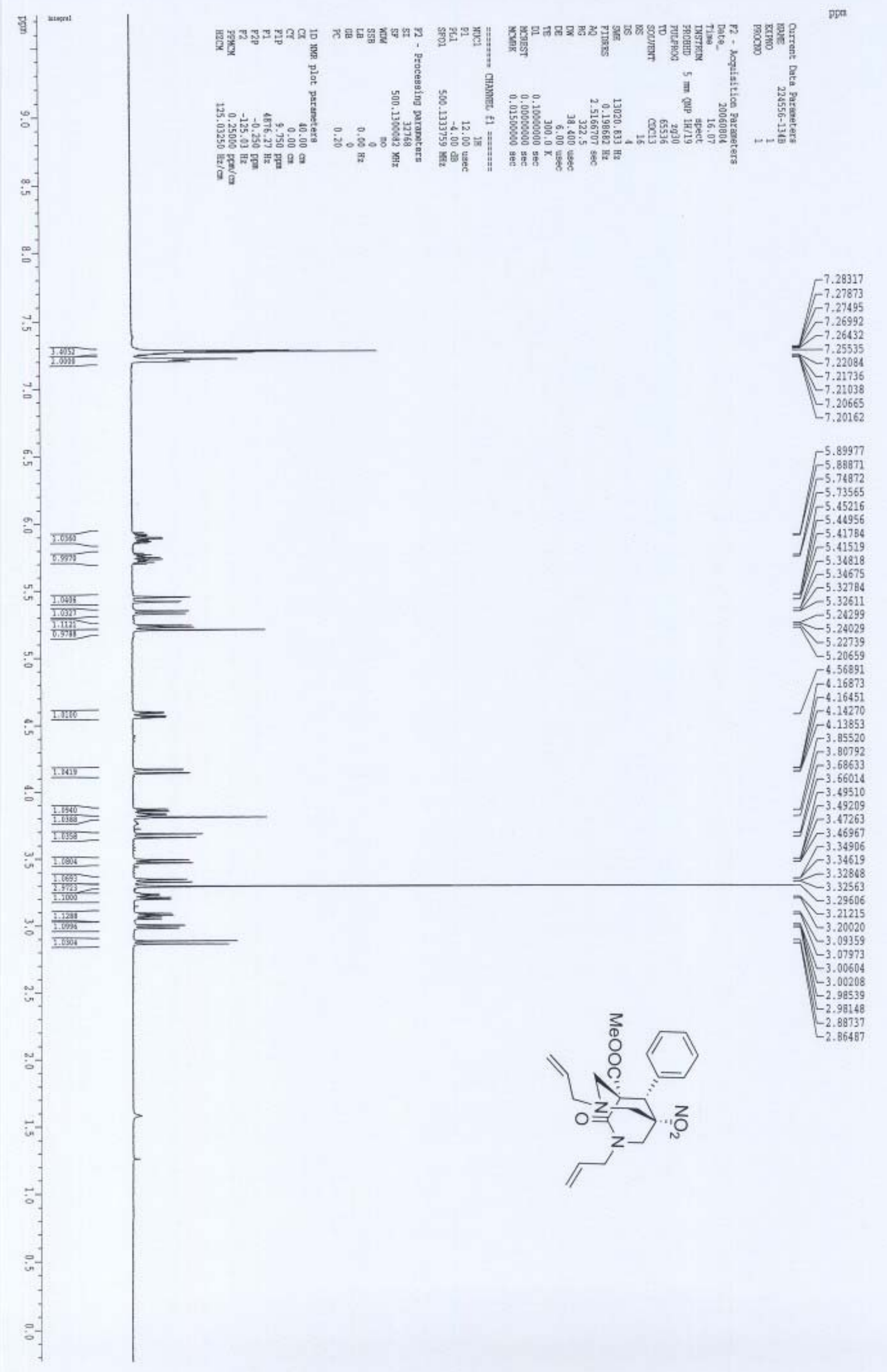




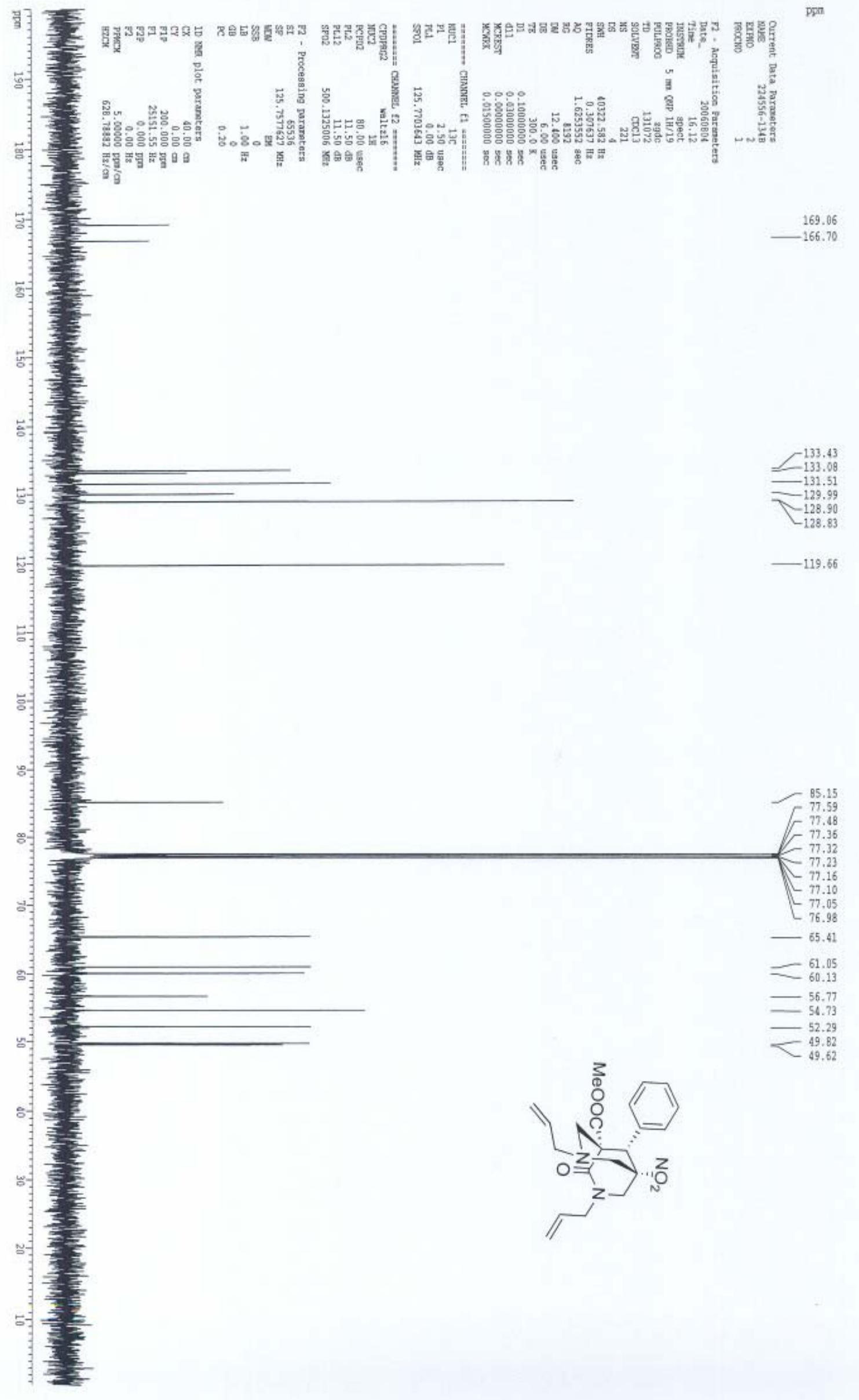




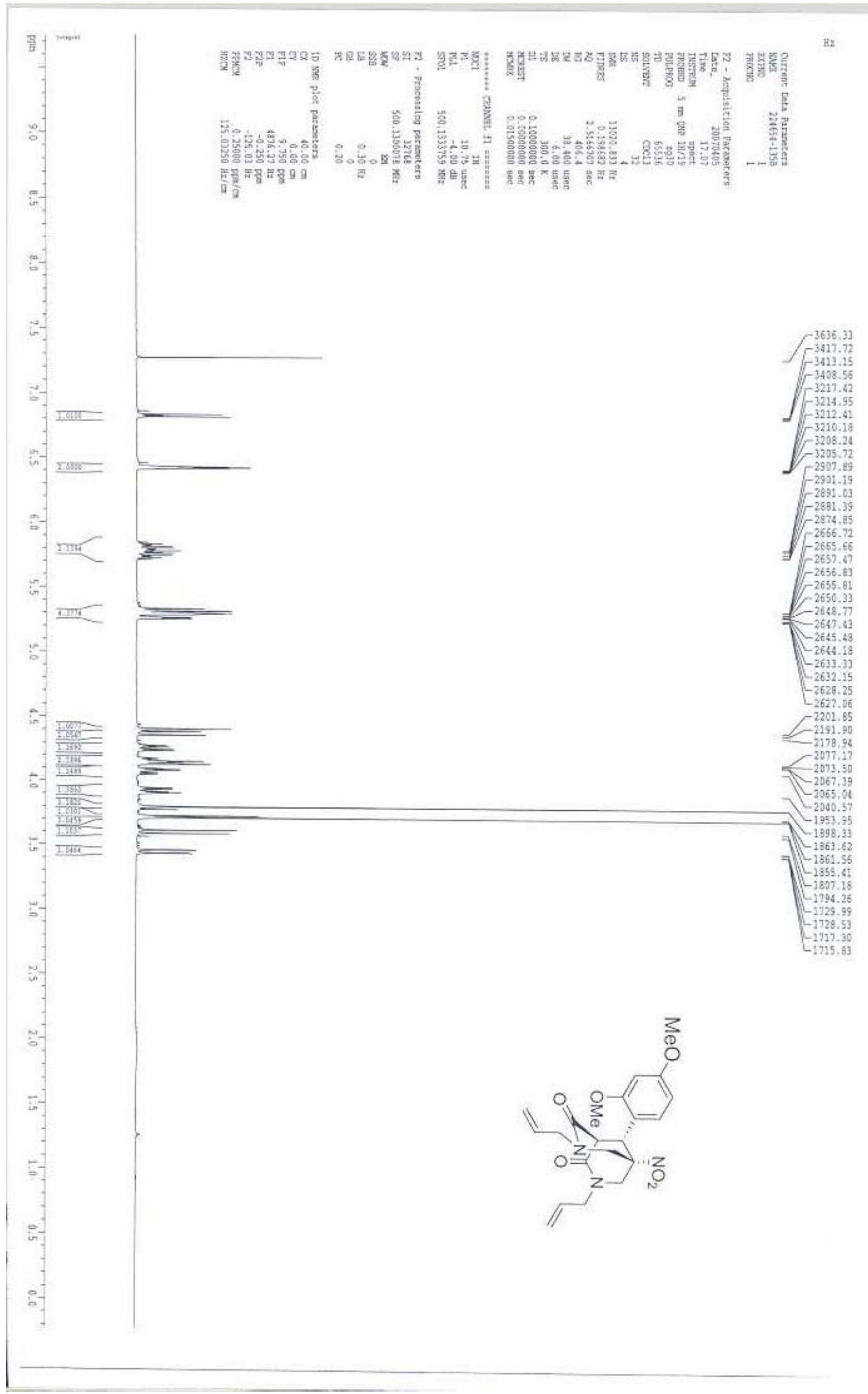




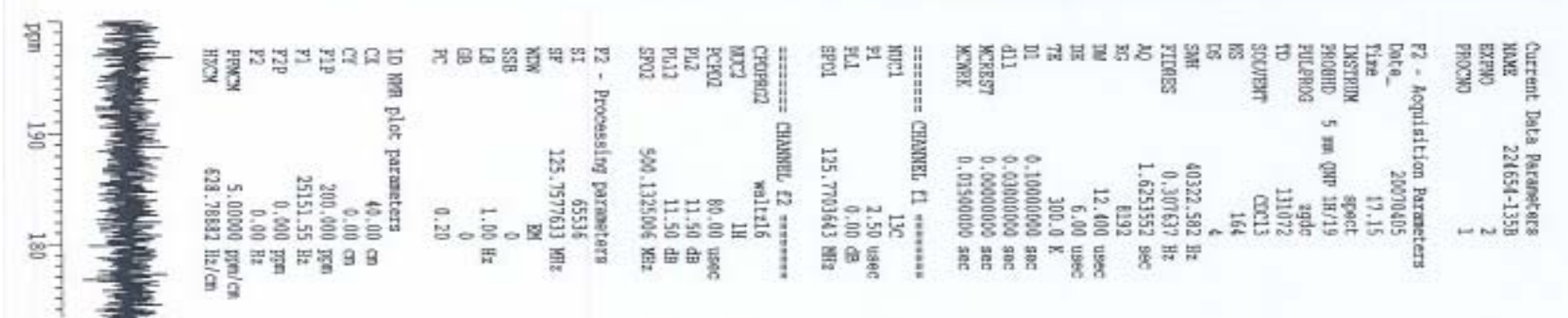

녕-

䟚
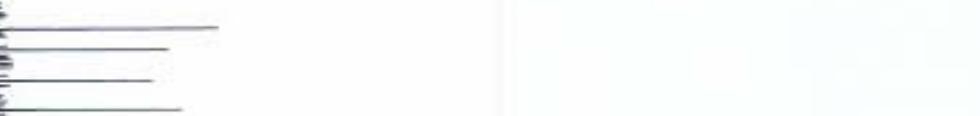

$-165.43$

$-163.81$ $-161.43$

형

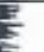

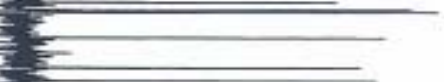

55.16

55.58

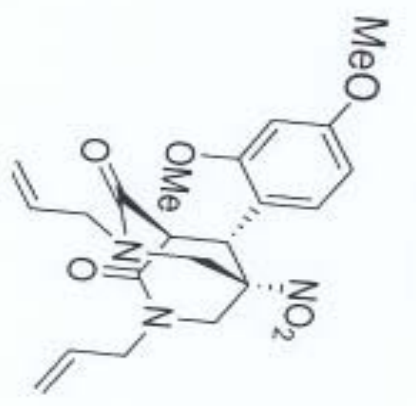




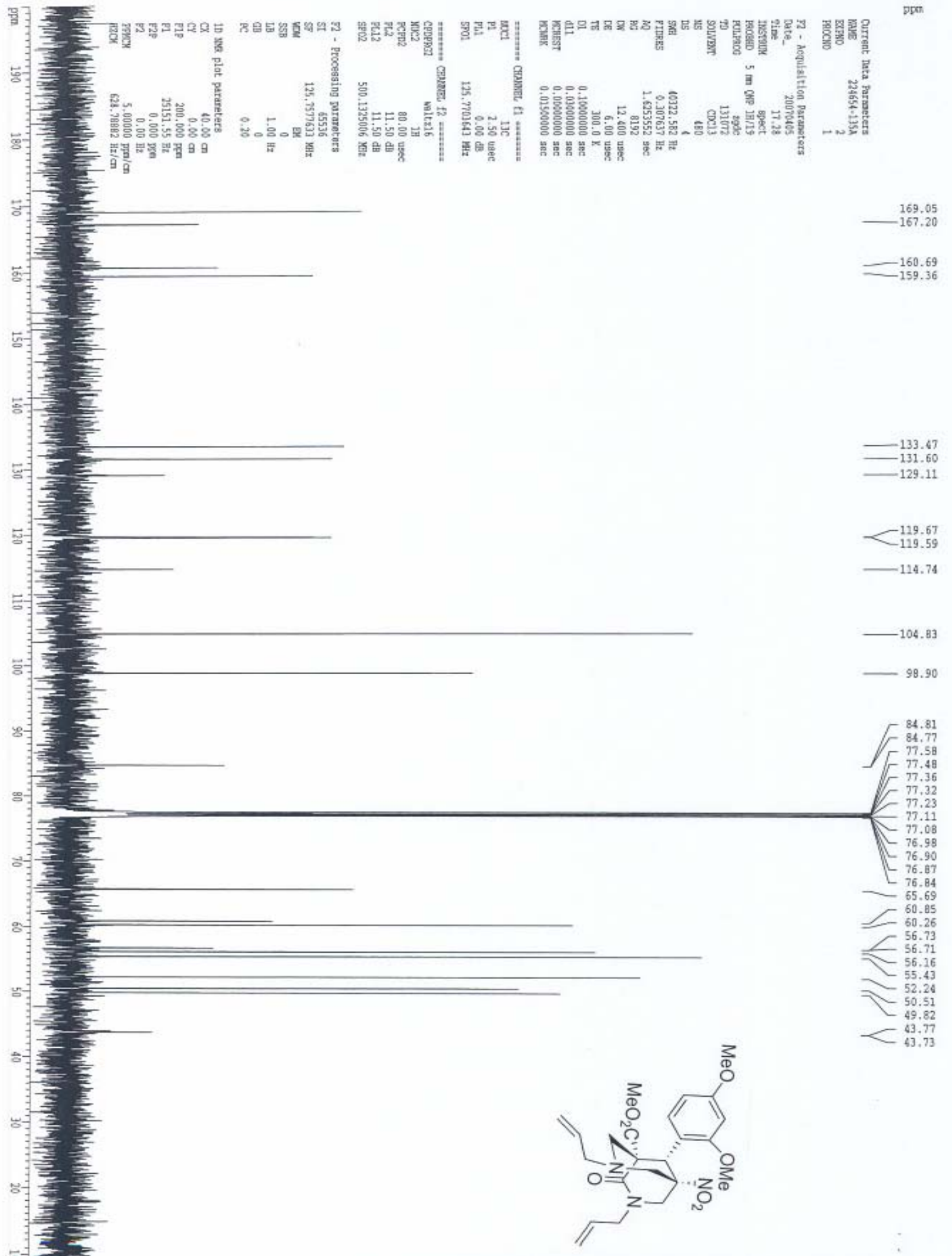




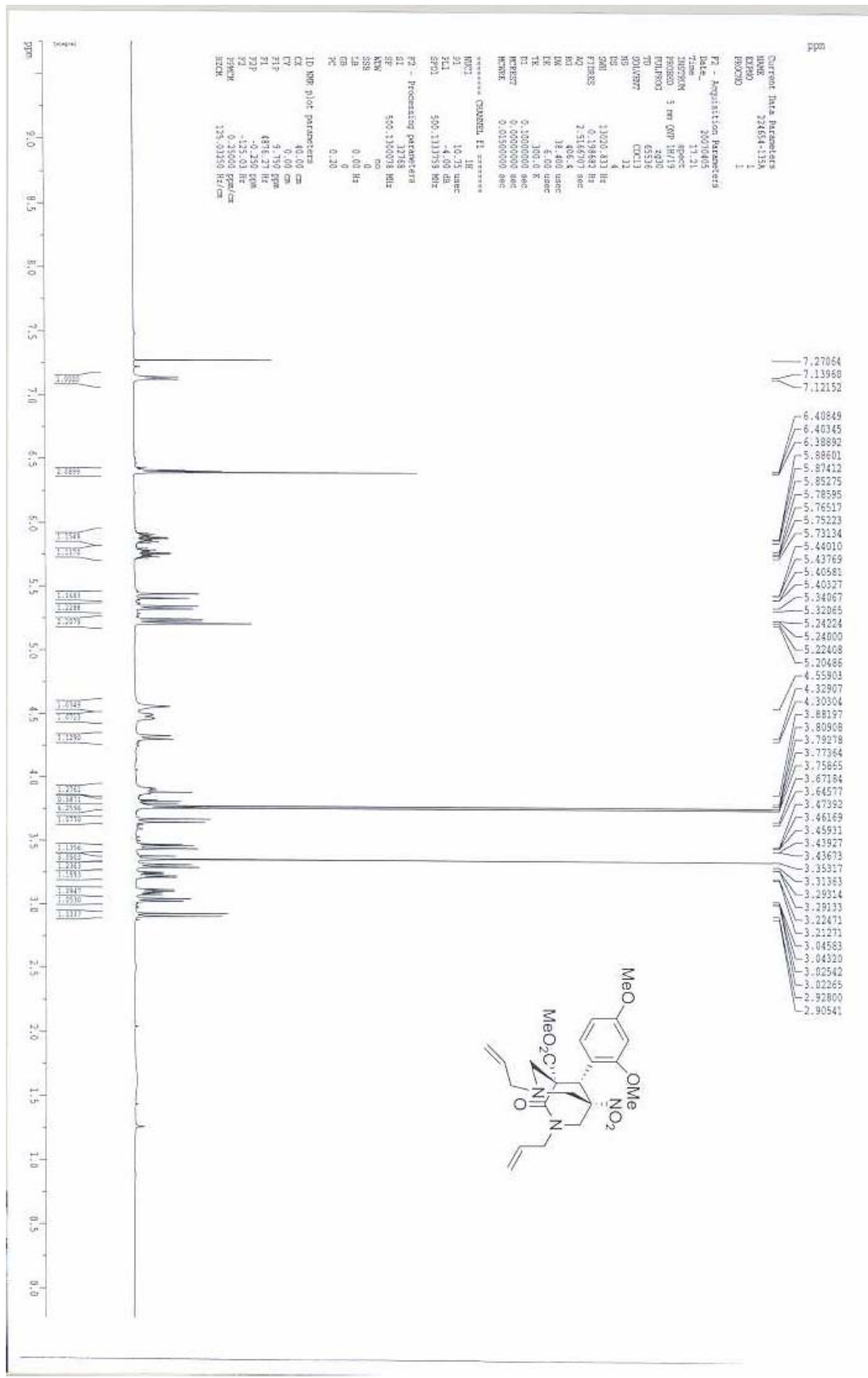




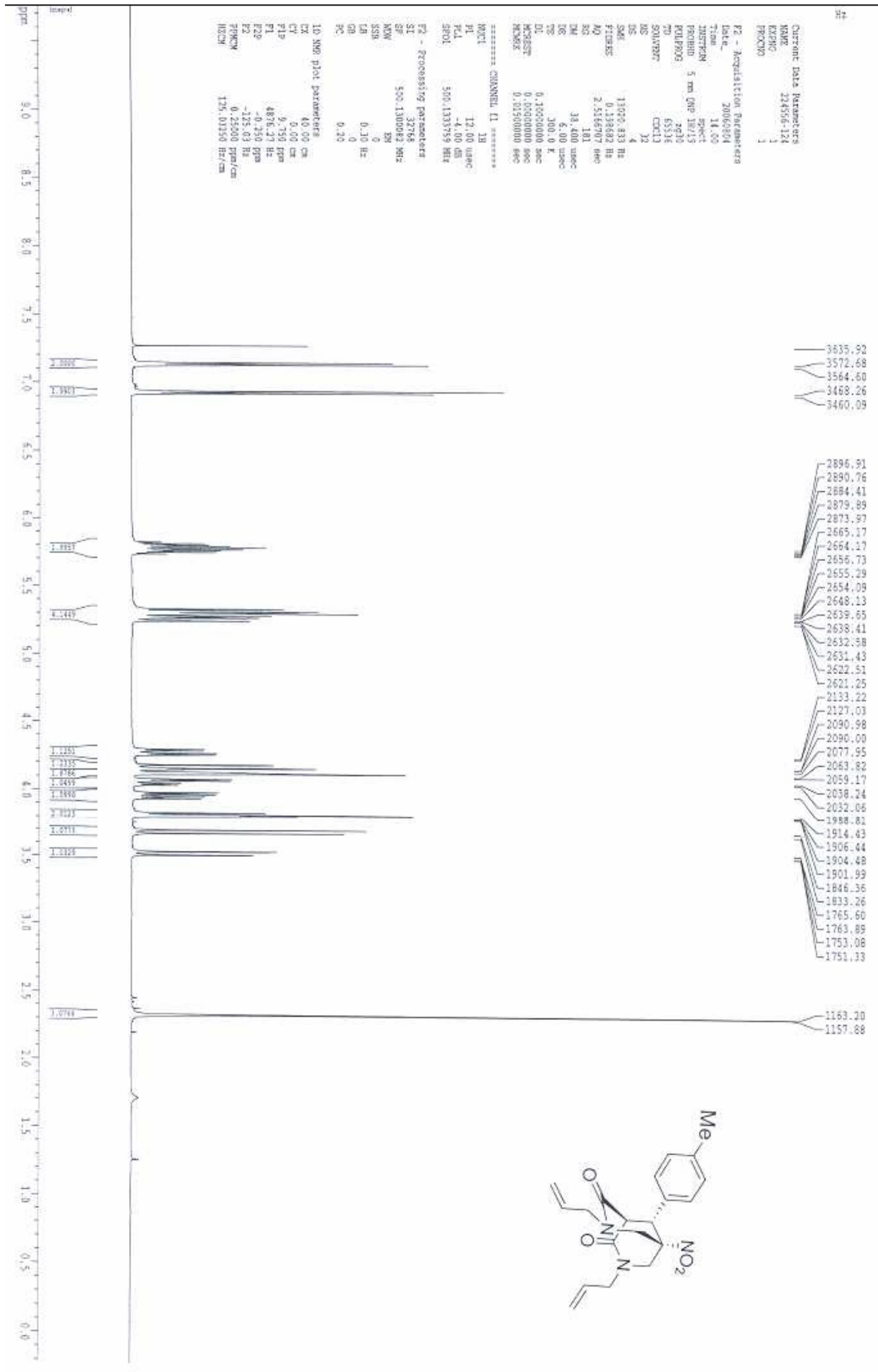



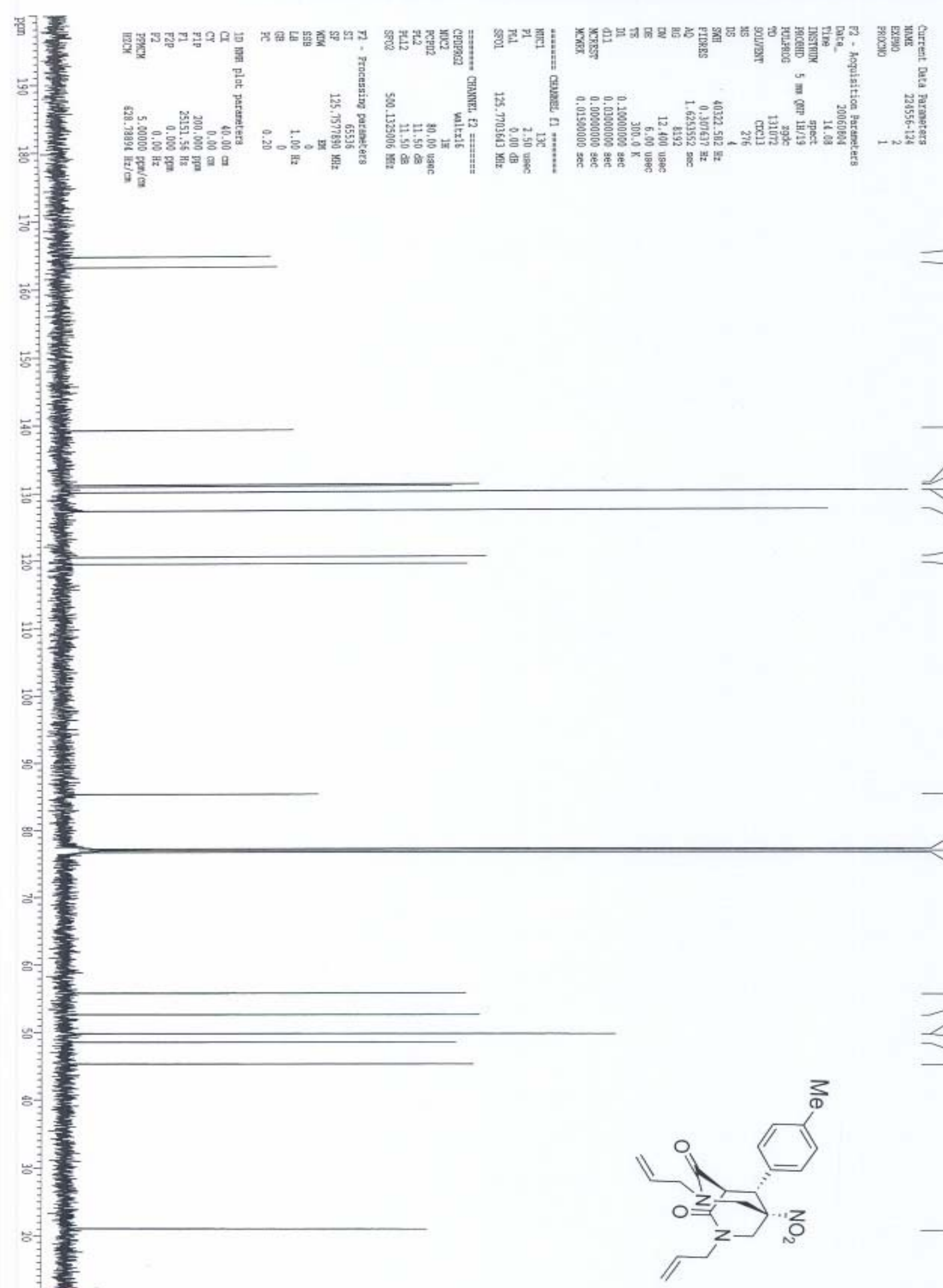


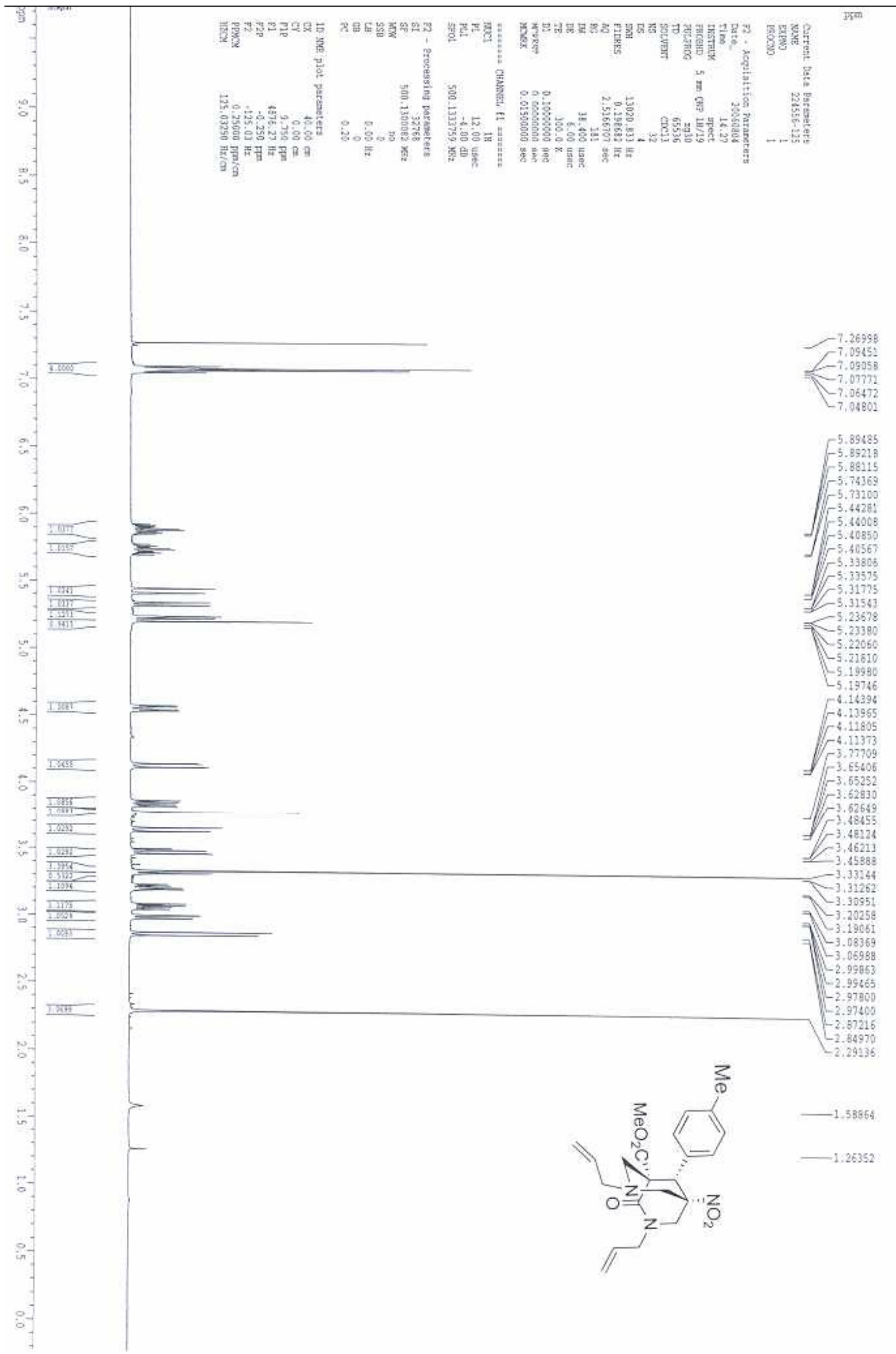




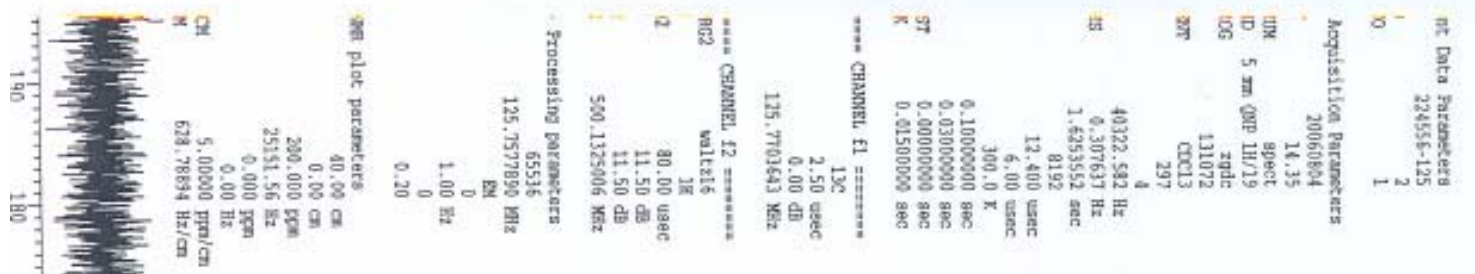

168.86 